\title{
IRREGULARITY OF HYPERGEOMETRIC SYSTEMS VIA SLOPES ALONG COORDINATE SUBSPACES
}

\author{
MATHIAS SCHULZE AND ULI WALTHER
}

\begin{abstract}
We study the irregularity sheaves attached to the $A$-hypergeometric $D$-module $M_{A}(\beta)$ introduced by I.M. Gel'fand et al. GGZ87 GZK89, where $A \in \mathbb{Z}^{d \times n}$ is pointed of full rank and $\beta \in \mathbb{C}^{d}$. More precisely, we investigate the slopes of this module along coordinate subspaces.

In the process we describe the associated graded ring to a positive semigroup ring for a filtration defined by an arbitrary weight vector $L$ on torusequivariant generators. To this end we introduce the $(A, L)$-umbrella, a cell complex determined by $A$ and $L$, and identify its facets with the components of the associated graded ring.

We then establish a correspondence between the full $(A, L)$-umbrella and the components of the $L$-characteristic variety of $M_{A}(\beta)$. We compute in combinatorial terms the multiplicities of these components in the $L$-characteristic cycle of the associated Euler-Koszul complex, identifying them with certain intersection multiplicities.

We deduce from this that slopes of $M_{A}(\beta)$ are combinatorial, independent of $\beta$, and in one-to-one correspondence with jumps of the $(A, L)$-umbrella. This confirms a conjecture of Sturmfels and gives a converse of a theorem of R. Hotta [Hot98, Ch. II, $\S 6.2$, Thm.]: $M_{A}(\beta)$ is regular if and only if $A$ defines a projective variety.
\end{abstract}

1991 Mathematics Subject Classification. 13N10,14M25,16W70.

Key words and phrases. hypergeometric, slope, characteristic variety, Euler-Koszul homology, toric, intersection multiplicity, D-module.

MS was supported by the Humboldt foundation.

UW was supported by the NSF under grant DMS 0555319, and by the NSA under grant H98230-06-1-0012. 


\section{Contents}

1. Introduction and overview

2. Filtrations on the toric ring

2.1. Torus action and toric ring

2.2. $L$-filtration on the toric ring

2.3. The $(A, L)$-umbrella

2.4. Monomials in the graded toric ideal

2.5. Homogeneity in the graded toric ideal

2.6. Minimal associated prime ideals

2.7. Index formula for multiplicities

2.8. Newton filtration and Cohen-Macaulayness

3. Characteristic variety of the hypergeometric system

3.1. Characteristic varieties and slopes 14

3.2. Hypergeometric system and candidate components 16

3.3. Existence of facet components 17

4. Characteristic cycle of the Euler-Koszul complex 19

4.1. Good filtrations and toric modules $\quad 19$

4.2. Characteristic cycle and Euler-Koszul homology 20

4.3. Euler-Koszul characteristic and intersection multiplicity 22

4.4. Rigidity of Euler-Koszul multiplicities 24

4.5. Euler-Koszul characteristic and volume 25

4.6. Generic Euler-Koszul homology 29

5. Projectivized hypergeometric systems 31

Appendix A. On spectral sequences 37

Acknowledgments

References

\section{INTRODUCTION AND OVERVIEW}

The solutions $f$ of an ordinary linear differential equation $P \bullet f(x)=0$ where

$$
P=p_{m}(x) \partial_{x}^{m}+p_{m-1}(x) \partial_{x}^{m-1}+\cdots+p_{1}(x) \partial_{x}+p_{0}(x)
$$

form a $\mathbb{C}$-vector bundle of dimension $m$ away from the zero locus of $p_{m}$. At $p_{m}\left(x_{0}\right)=0$, two types of singularities may occur: at a regular singular point $x_{0}$, the (multivalued) solutions have polynomial growth for $x \rightarrow x_{0}$ while in all other cases $x_{0}$ is called irregular. By Fuchs' Theorem, $P$ is regular at the origin if and only if the Newton polygon $\mathrm{N}(P)$ of $P$ is a quadrant (see [nc44]). The slopes (or critical indices) of $P$ are just the slopes of $\mathrm{N}(P)$; they represent a refined notion of irregularity (see Lau85]) and indicate the growth of solutions near the critical point. Regularity at $x_{0}$ is equivalent to equality of formal and convergent solutions at $x_{0}$.

The concept of regularity in higher dimension is considerably more involved. Denote by $\mathcal{O}_{X}$ the structure sheaf of the complex manifold $X$ and by $\mathcal{O}_{\widehat{X \mid Y}}$ the completion of $\mathcal{O}_{X}$ along the submanifold $Y$. For any coherent $\mathcal{D}_{X}$-module $\mathcal{M}, \mathrm{Z}$. Mebkhout introduced the irregularity complex $\mathcal{I r r}_{Y}(M)=\mathbf{R} \mathcal{H}_{\mathcal{D o m}_{X}}\left(\mathcal{M}, \mathcal{O}_{\widehat{X \mid Y}} / \mathcal{O}_{X \mid Y}\right), \mathcal{O}_{X \mid Y}$ 
being the restriction of $\mathcal{O}_{X}$ to $Y$ Meb89. This intrinsically analytic notion is inspired by earlier work of $\mathrm{B}$. Malgrange on regularity in the univariate case [Mal74, but quite difficult to use.

On the other hand, Y. Laurent Lau87, gave a generalization for the concept of a slope to the multivariate case based on more algebraic methods. With $X, Y$ as above, let $V$ be the Kashiwara-Malgrange filtration along $Y$, let $F$ be the order filtration, and put $L=p F+q V$. Then the rational number $p / q>0$ is a slope of $\mathcal{M}$ along $Y$ if the $L$-characteristic variety $\operatorname{Ch}^{L}(\mathcal{M})=\operatorname{supp}\left(\operatorname{gr}^{L}(\mathcal{M})\right) \subseteq T^{*} X$ jumps (is not locally constant) at $p / q$.

The theorem of Fuchs generalizes to the multivariate case: by the analyticalgebraic comparison theorem for slopes [LM99, Thm. 2.4.2], $\mathcal{M}$ has no slopes along $Y$ precisely if $\operatorname{Irr}_{Y}(\mathcal{M})$ is exact. In fact, the slopes agree with the jumps of the Gevrey filtration on the irregularity complex which provide a measure of growth for the solutions of $\mathcal{M}$ near $Y$.

All slopes of $\mathcal{M}$ along $Y$ are rational and there are only a finite number of them, (see Lau87]). A. Assi, F. Castro, and M. Granger [ACJG96 developed a Gröbner basis algorithm to compute slopes of algebraic $D$-modules using the algebraic counterparts of $F, V$, and $L$ on the Weyl algebra $D$. In the process they proved a comparison theorem: slopes of modules over the Weyl algebra can be computed without leaving the algebraic category, where rationality and finiteness follow from the existence of the Gröbner fan (see ACJG00). Explicit formulæ for slopes of $D$-modules are very rare. The purpose of this article is to describe the slopes of $A$-hypergeometric $D$-modules.

I.M. Gel'fand, M.I. Graev, M.M. Kapranov and A.V. Zelevinskiu GGZ87, GZK89 defined a class of $D$-modules that includes as particular cases the differential systems satisfied by the classical hypergeometric functions of Gauß, Appell, and others. These $A$-hypergeometric, or GKZ (after Gel'fand, Kapranov, and Zelevinskiı̌), systems are special cases of the equivariant $D$-modules of R. Hotta and M. Kashiwara HK84, Hot98. A $d \times n$ integer matrix $A$ defines an action of a $d$-torus $\mathbb{T}:=\left(\mathbb{C}^{*}\right)^{d}$ on $T_{0}^{*} \mathbb{C}^{n}=\mathbb{C}^{n}$. Our general hypothesis is that $\mathbb{N} A$ is a positive semigroup with $\mathbb{Z} A=\mathbb{Z}^{d}$. The closure of the orbit through $(1, \ldots, 1)$ is defined by the toric ideal $I_{A} \subseteq \mathbb{C}[\partial]=: R$ where $\partial:=\partial_{1}, \ldots, \partial_{n}$ and $S_{A}:=R / I_{A}=\mathbb{C}[\mathbb{N} A]$ is the associated semigroup ring. The Euler vector fields $E=\left(E_{1}, \ldots, E_{d}\right)$ are the pushforwards to this orbit of the Lie algebra generators $t_{1} \partial_{1}, \ldots, t_{d} \partial_{d}$ of $\mathbb{T}$. The $A$-hypergeometric system $M_{A}(\beta)$, depending on the Lie algebra character $\beta \in \mathbb{C}^{d}$, is the $D$-module defined by $I_{A}$ and the Euler operators $E-\beta$. It arises in various situations in algebraic geometry such as in the theory of toric residues (see CDS01]), the study of hyperplane arrangements (see OT01]), and in the Picard-Fuchs equations governing the variation of Hodge structures for Calabi-Yau toric hypersurfaces (see CK99]).

By a theorem of R. Hotta [Hot98, Ch. II, $\S 6.2$, Thm.], homogeneous $A$-hypergeometric systems are regular and hence have no slopes. In dimension one and in codimension one, slopes of $M_{A}(\beta)$ were studied by F. Castro and N. Takayama CJT03. and M. Hartillo [HH03, HH05. Cohen-Macaulayness of the toric rings in question makes these cases comparatively tractable. In our general situation, a key tool is a (generalization of a) computation by A. Adolphson Ado94 identifying candidate components of the $F$-characteristic variety with the set of faces not containing 0 of the convex hull $\Delta_{A}^{F}$ of 0 and the columns $\mathbf{a}_{1}, \ldots, \mathbf{a}_{n}$ of $A$. In Section 2, $L$ is the 
filtration on $R$ defined by an arbitrary weight vector on $\partial_{1}, \ldots, \partial_{n}$. We introduce in Definition 2.7 the $(A, L)$-polyhedron $\Delta_{A}^{L}$ as the convex hull in projective space of 0 and all $\mathbf{a}_{i}^{L}:=\mathbf{a}_{i} / \operatorname{deg}^{L}\left(\partial_{i}\right)$ for $i=1, \ldots, n$. Its faces not containing zero form the $(A, L)$-umbrella $\Phi_{A}^{L}$, a combinatorial object independent of $\beta$ that encodes all information regarding the $L$-characteristic variety of $M_{A}(\beta)$.

Figure 1. Some $(A, L)$-umbrellas. (Shaded $\Delta_{A}^{L}$ with fat boundary $\Phi_{A}^{L}$.)
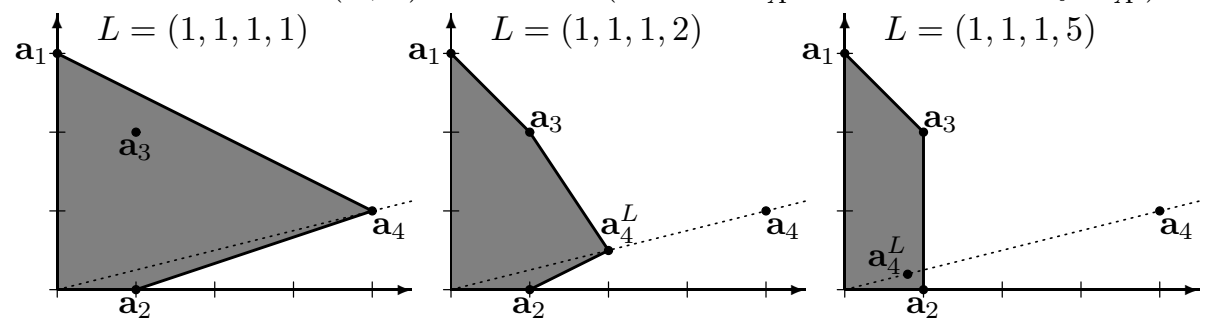

Figure 1 shows the $(A, L)$-umbrella in the example

$$
A=\left(\begin{array}{llll}
0 & 1 & 1 & 4 \\
3 & 0 & 2 & 1
\end{array}\right)
$$

for the family of filtrations $L=(1,1,1, t)$ for three parameters resulting in combinatorially different umbrellas. In Theorem 2.14 we identify $\Phi_{A}^{L}$ with both the $\mathbb{Z}^{d}$-graded spectrum of the $L$-graded toric ring $S_{A}^{L}:=\operatorname{gr}^{L}\left(S_{A}\right)$ and the collection of torus orbits in $\operatorname{Spec}\left(S_{A}^{L}\right)$. In Theorem 2.20, we characterize Cohen-Macaulayness of $S_{A}^{L}$ by the corresponding property of its subrings generated by facet cones.

In Section 3, we extend $L$ to the Weyl algebra $D=\mathbb{C}[x]\langle\partial\rangle$ in the variables $x:=$ $x_{1}, \ldots, x_{n}$ in such a way that $E$ is $L$-homogeneous and $W:=\operatorname{gr}^{L}(D)$ is a polynomial ring. The family of filtrations in Figure 1 may be viewed as the restriction of $L=F+q V$ to $R$ for the $V$-filtration along $x_{4}$ for $q=0,1,4$. In Proposition 3.8 we show that all components of the $L$-characteristic variety of $M_{A}(\beta)$ correspond to faces $\tau \in \Phi_{A}^{L}$ : each is the closure $\bar{C}_{A}^{\tau}$ of the conormal space $C_{A}^{\tau}$ to a torus orbit in $\operatorname{Spec}\left(S_{A}^{L}\right)$. The facet components correspond to orbits in the smooth, and hence Cohen-Macaulay, locus of $S_{A}^{L}$ on which the $L$-symbols of the Euler vector fields form a regular sequence. From this we conclude in Proposition 3.10 that the facet components actually occur and their multiplicity is given by an index formula independent of the parameter $\beta$. This shows in particular that $p / q$ is a slope of $M_{A}(\beta)$ at the origin whenever $\Phi_{A}^{p F+q V}$ jumps at $p / q$. We thus obtain a converse to Hotta's theorem in Corollary 3.16 regular $A$-hypergeometric systems are homogeneous.

Since orbits to nonfacets may be outside the Cohen-Macaulay locus of $S_{A}^{L}$, we consider in Section 4 the full Euler-Koszul complex $K_{A, \bullet}\left(S_{A} ; \beta\right)$ from MMW05. In order to apply methods of homological algebra, we discuss the basics of good $L$-filtrations on $R$ - and $D$-modules. Using the spectral sequence for the $L$-filtration on $K_{A, \bullet} \cdot\left(S_{A} ; \beta\right)$, we identify its $L$-characteristic cycle with the intersection cycle between $S_{A}^{L}$ and the $L$-graded Euler ideal $\operatorname{gr}^{L}(\langle E\rangle)$. In Theorem 4.11, we use Serre's Intersection Theorem [Ser65, Ch. V, §C.1, Thm. 1] to show that the $L$ characteristic variety of $K_{A, \bullet}\left(S_{A} ; \beta\right)$ contains all candidate components. To show that this also holds for $M_{A}(\beta)=H_{0}\left(K_{A, \bullet}\left(S_{A} ; \beta\right)\right)$ we use in Theorem 4.16 results 
from MMW05 and an induction argument on toric modules. In particular (see Corollary 4.17), the components of the $L$-characteristic variety of $M_{A}(\beta)$ are in one-to-one correspondence to the faces in the $(A, L)$-umbrella $\Phi_{A}^{L}$. It follows (see Corollary 4.18) that the slopes of $M_{A}(\beta)$ along coordinate subspaces at the origin correspond exactly to jump parameters $p / q$ of $\Phi_{A}^{p F+q V}$, confirming and extending a conjecture by B. Sturmfels.

The holonomic $\operatorname{rank} \operatorname{rk}(M):=\operatorname{dim}_{\mathbb{C}(x)}\left(M \otimes_{\mathbb{C}[x]} \mathbb{C}(x)\right)$ of a $D$-module $M$ is the dimension of the $\mathbb{C}$-vector space of its solution space near a regular point. It is a classical result (see GZK89, Ado94) that for generic $\beta$ the rank of $M_{A}(\beta)$ equals the volume of the convex hull $\Delta_{A}^{F}$ of 0 and $\mathbf{a}_{1}, \ldots, \mathbf{a}_{n}$ where the volume of the unit simplex is normalized to 1 . The exceptional set $\mathcal{E}(A)$ of $A$ is the set of rankjumping parameters: $\beta \in \mathcal{E}(A)$ precisely if the rank of $M_{A}(\beta)$ exceeds the volume. By [MMW05, $\mathcal{E}(A)$ is a finite subspace arrangement. In Theorem 4.21 we give a general index/volume formula for the multiplicity $\mu_{A}^{L, \tau}$ of $\bar{C}_{A}^{\tau}, \tau \in \Phi_{A}^{L}$, in the $L$-characteristic cycle of $K_{A, \bullet}\left(S_{A} ; \beta\right)$. We show then that for non-rank-jumping parameter $\beta$, or if $\tau$ is a facet, the number $\mu_{A}^{L, \tau}$ equals the multiplicity $\mu_{A, 0}^{L, \tau}(\beta)$ of $\bar{C}_{A}^{\tau}$ in the $L$-characteristic cycle of $M_{A}(\beta)$. From MMW05 it is known that the $\operatorname{rank} \mu_{A, 0}^{F, \emptyset}(\beta)$ of $M_{A}(\beta)$ is upper semicontinuous in the parameter $\beta$. Theorem 4.28 generalizes a weaker statement to all $L$ and $\tau \in \Phi_{A}^{L}$ : the multiplicity $\mu_{A, 0}^{L, \tau}(\beta)$, $\tau \in \Phi_{A}^{L}$, is always minimal at generic $\beta$. We conjecture that $\mu_{A, 0}^{L, \tau}(\beta)$ is upper semicontinuous in $\beta$.

In the last section, we generalize results of the previous sections to a natural extension of $M_{A}(\beta)$ to the projective closure $\left(\mathbb{P}_{\mathbb{C}}^{1}\right)^{n}$.

\section{Filtrations ON The tORIC RING}

\subsection{Torus action and toric ring.}

Notation 2.1. By $\mathbb{Q}_{+}$we mean the nonnegative rational numbers and we include 0 in $\mathbb{N}$.

Let $A=\left(a_{i, j}\right) \in \mathbb{Z}^{d \times n}$ be an integer matrix of rank $d$ whose columns $\mathbf{a}_{1}, \ldots, \mathbf{a}_{n} \in$ $\mathbb{Z}^{d}$ are nonzero. We assume that $\mathbb{N} A$ is a positive (see [BH93, $\left.\S 6.1\right]$ ) (or pointed, see [MMW05, §1]) semigroup with $\mathbb{Z} A=\mathbb{Z}^{d}$. We write $\tau \subseteq A$ if $\tau$ is a subset of the column set of $A$ and consider it both as a submatrix of $A$ and a subset of the set of column indices $\{1, \ldots, n\}$. Then the dimension $\operatorname{dim}(\tau)$ is $\operatorname{dim}_{\mathbb{Q}}(\mathbb{Q} \tau)-1$. For a vector or collection with index set $\{1, \ldots, n\}$, a lower index $\tau$ denotes the subvector or subcollection with indices in $\tau$. We abbreviate $\bar{\tau}:=\{1, \ldots, n\} \backslash \tau$. For any set $\tau$, its cardinality is denoted $|\tau|$.

We shall frequently denote by $\bar{C}$ the Zariski closure of a set $C$.

For $\mathbf{u} \in \mathbb{Z}^{n}$ define $\mathbf{u}_{+}$by $\left(\mathbf{u}_{+}\right)_{j}=\max \left(0, \mathbf{u}_{j}\right)$ and put $\mathbf{u}_{-}=\mathbf{u}_{+}-\mathbf{u}$. For $\mathbf{u}, \mathbf{v} \in \mathbb{N}^{n}$ write $\min (\mathbf{u}, \mathbf{v})$ for the vector whose $j$-th entry is $\min \left(\mathbf{u}_{j}, \mathbf{v}_{j}\right)$. For any vector $\mathbf{u}$ we mean by $\mathbf{u}>0$ that $\mathbf{u}$ is componentwise positive: $u_{i}>0$ for all $i$.

The base space in this note is $X:=\operatorname{Spec}(\mathbb{C}[x])=\mathbb{C}^{n}$ where $x:=x_{1}, \ldots, x_{n}$. Let $R:=\mathbb{C}[\partial]$ be the polynomial ring in $n$ variables $\partial:=\partial_{1}, \ldots, \partial_{n}$. Identifying $\partial_{i}$ with the partial derivation $\partial / \partial x_{i}, \operatorname{Spec}(R)$ becomes the conormal space $T_{0}^{*} X$ of $X$ at 0 . The $d$-torus $\mathbb{T}:=\left(\mathbb{C}^{*}\right)^{d}=\operatorname{Spec}\left(\mathbb{C}\left[t_{1}^{ \pm 1}, \ldots, t_{d}^{ \pm 1}\right]\right)$ with coordinates $t:=t_{1}, \ldots, t_{d}$ acts on $\operatorname{Spec}(R)$

$$
(t, \xi) \longmapsto t \cdot \xi:=\left(t^{\mathbf{a}_{1}} \xi_{1}, \ldots, t^{\mathbf{a}_{n}} \xi_{n}\right)
$$


This induces a $\mathbb{Z}^{d}$-grading on $R$ by $\operatorname{deg}\left(\partial_{i}\right)=\mathbf{a}_{i}$. For a $\mathbb{Z}^{d}$-graded $R$-module $N$, we denote by $\operatorname{deg}(N)$ the set of its $\mathbb{Z}^{d}$-degrees.

Definition 2.2. We denote the orbit $\mathbb{T} \cdot \xi$ through $\xi \in T_{0}^{*} X$ by $\operatorname{Orb}(\xi)$. Let $\tau$ be a subset of columns of $A$. We define $\mathbf{1}_{A}^{\tau} \in\{0,1\}^{n}$ by

$$
\left(\mathbf{1}_{A}^{\tau}\right)_{j}:=\left\{\begin{array}{lll}
1 & \text { if } & \mathbf{a}_{j} \in \tau, \\
0 & \text { if } & \mathbf{a}_{j} \notin \tau,
\end{array}\right.
$$

and denote by $O_{A}^{\tau}$ the orbit of $\mathbf{1}_{A}^{\tau}$.

The toric ideal $I_{\tau} \subseteq R_{\tau}:=\mathbb{C}\left[\partial_{\tau}\right]$ of $\tau$ is the $\mathbb{Z}^{d}$-graded prime ideal generated by all $\square_{\mathbf{u}}=\partial^{\mathbf{u}_{+}}-\partial^{\mathbf{u}_{-}}$where $\mathbf{u} \in \mathbb{Z}^{|\tau|}$ such that $\tau \cdot \mathbf{u}=0$. We set $I_{A}^{\tau}:=R I_{\tau}+J_{\tau}$ where $J_{\tau}$ is the $R$-ideal generated by $\left\{\partial_{i} \mid i \notin \tau\right\}$. Based on the following lemma, the semigroup ring of $\tau$ is

$$
S_{\tau}:=\mathbb{C}[\mathbb{N} \tau]=\bigoplus_{\mathbf{a} \in \mathbb{N} \tau} \mathbb{C} \cdot t^{\mathbf{a}}=\sum_{\mathbf{u} \in \mathbb{N}|\tau|} \mathbb{C} \cdot\left(\partial_{\tau}^{\mathbf{u}} \bmod I_{\tau}\right) \subseteq S_{A},
$$

The normalization of $S_{A}$ is the Cohen-Macaulay ring $\tilde{S}_{A}=\bigoplus_{\mathbf{a} \in\left(\mathbb{Q}_{+} A \cap \mathbb{Z}^{d}\right)} \mathbb{C} \cdot t^{\mathbf{a}}$.

Lemma 2.3. For $\tau \subseteq A, I_{A}^{\tau}=\left\{f \in R \mid f\left(O_{A}^{\tau}\right)=0\right\}$ and $S_{\tau}=R_{\tau} / I_{\tau}=R / I_{A}^{\tau}$.

2.2. $L$-filtration on the toric ring. Let $L=\left(L_{\partial_{1}}, \ldots, L_{\partial_{n}}\right) \in \mathbb{Q}^{n}$ be a weight vector. It induces an increasing filtration $L$ of $\mathbb{C}$-vector spaces on $R$ via $\left[\partial^{\mathbf{u}} \in\right.$ $\left.L_{k} R\right] \Leftrightarrow[L \cdot \mathbf{u} \leq k]$. Note that $L$ has a rational discrete index set. If $f \in L_{k} R \backslash L_{<k} R$ then $k=: \operatorname{deg}^{L}(f)$ is the $L$-degree of $f$. Let $\sigma^{L}: R \rightarrow \operatorname{gr}^{L}(R)$ be the $L$-symbol map defined by $\sigma^{L}(f)=f \bmod L_{<k} R$ if $\operatorname{deg}^{L}(f)=k$. An element $f \in R$ is $L$-homogeneous if $f=\sum_{L \cdot \mathbf{u}=k} f_{\mathbf{u}} \partial^{\mathbf{u}}$ for some $k \in \mathbb{Q}$ where $f_{\mathbf{u}} \in \mathbb{C}$. By abuse of notation, we identify $R$ and $\operatorname{gr}^{L}(R)$ via the $\mathbb{C}$-linear isomorphism induced by $f \mapsto \sigma^{L}(f)$ for $L$-homogeneous $f$ in right-normal form.

For any $\tau \subseteq A, L$ induces a filtration on $S_{\tau}$ by

$$
L_{k} S_{\tau}:=\sum_{\operatorname{deg}^{L}\left(\partial_{\tau}^{\mathbf{u}}\right) \leq k} \mathbb{C} \cdot\left(\partial_{\tau}^{\mathbf{u}} \bmod I_{\tau}\right)
$$

With $I_{\tau}^{L}$ denoting the $\mathbb{Z}^{d}$-graded ideal $\operatorname{gr}^{L}\left(I_{\tau}\right)$, we abbreviate

$$
S_{\tau}^{L}:=\operatorname{gr}^{L}\left(S_{\tau}\right) \cong R / I_{\tau}^{L} .
$$

The following is a mild extension of [Stu96, Cor. 4.4] (see also [Ado94, Lem. 4.11]).

Lemma 2.4. One has the identity $I_{A}^{L}=R\left\langle\sigma^{L}\left(\square_{\mathbf{u}}\right) \mid \mathbf{u} \in \mathbb{Z}^{n}, A \cdot \mathbf{u}=0\right\rangle$.

Proof. Generators of $I_{A}^{L}$ are the $L$-symbols of a reduced Gröbner basis of $I_{A}$ and can be computed by Buchberger's algorithm. But each S-pair and reduction step of the algorithm preserves the generators $\left\{\square_{\mathbf{u}} \mid \mathbf{u} \in \mathbb{Z}^{n} \cap \operatorname{ker}(A)\right\}$ : If $\mathbf{u}^{\prime}+\mathbf{u}_{+}=\mathbf{v}^{\prime}+\mathbf{v}_{+}$ where $\mathbf{u}, \mathbf{v} \in \mathbb{Z}^{n} \cap \operatorname{ker}(A)$ and $\mathbf{u}^{\prime}, \mathbf{v}^{\prime} \in \mathbb{N}^{n}$ then

$$
A\left(\mathbf{v}^{\prime}+\mathbf{v}_{-}\right)=A\left(\mathbf{v}^{\prime}+\mathbf{v}_{+}\right)=A\left(\mathbf{u}^{\prime}+\mathbf{u}_{+}\right)=A\left(\mathbf{u}^{\prime}+\mathbf{u}_{-}\right) .
$$

and

$$
\partial^{\mathbf{u}^{\prime}} \square_{\mathbf{u}}-\partial^{\mathbf{v}^{\prime}} \square_{\mathbf{v}}=\partial^{\mathbf{v}^{\prime}+\mathbf{v}_{-}}-\partial^{\mathbf{u}^{\prime}+\mathbf{u}_{-}}=\partial^{\min \left(\mathbf{u}^{\prime}+\mathbf{u}_{-}, \mathbf{v}^{\prime}+\mathbf{v}_{-}\right)} \square_{\mathbf{v}^{\prime}+\mathbf{v}_{-}-\mathbf{u}^{\prime}-\mathbf{u}_{-} .} .
$$

Note that this argument is valid also for $L \ngtr 0$ where Buchberger's algorithm with (de-)homogenization is used. 
In this section we study the geometry of $S_{A}^{L}$ in terms of $A$ and $L$. Since the Gröbner fan of any $R$-ideal is defined over $\mathbb{Q}$, the study of real weights can be reduced to the case $L \in \mathbb{Q}^{n}$ (see [MS05, §7.4] for a discussion in the case where $L>0$ defines a term order).

2.3. The $(A, L)$-umbrella. We consider the embedding of the affine space $\mathbb{Q}^{d} \supseteq A$ into the rational projective $d$-space

$$
\mathbb{P}_{\mathbb{Q}}^{d}=\mathbb{P}_{\mathbb{Q}}\left(\mathbb{Q}^{d} \times \mathbb{Q}\right)
$$

via the map $q \mapsto(q: 1)$. Denote $\infty:=\mathbb{P}_{\mathbb{Q}}^{d} \backslash \mathbb{Q}^{d}$ the hyperplane at infinity.

In $\mathbb{P}_{\mathbb{Q}}^{d}$, any two distinct points $\mathbf{a}, \mathbf{b} \in \mathbb{P}_{\mathbb{Q}}^{d}$ are joined by two line segments. If $H$ is a hyperplane in $\mathbb{P}_{\mathbb{Q}}^{d}$ containing neither a nor $\mathbf{b}$ then there is a unique line segment joining the points and not meeting $H$. This is exactly the convex hull of $\mathbf{a}$ and $\mathbf{b}$ in the affine space $\mathbb{P}_{\mathbb{Q}}^{d} \backslash H$.

Definition 2.5. Let $H \subseteq \mathbb{P}_{\mathbb{Q}}^{d}$ be a hyperplane and let $U_{H}:=\mathbb{P}_{\mathbb{Q}}^{d} \backslash H$ be its complement. For $B \subseteq U_{H}$, the convex hull of $B$ relative to $H$ is the set $\operatorname{conv}_{H}(B)$ defined as the convex hull of $B$ in the affine space $U_{H}$.

Note that, for varying $H, \operatorname{conv}_{H}(B)$ changes exactly when $H$ is moved through a point of $B$. Within $\mathbb{Q}^{d}$, elements of convex hulls are linear combinations with nonnegative coefficients that add to unity. Convex hulls relative to $H \neq \infty$, with coordinates from $\mathbb{Q}^{d} \subseteq \mathbb{P}_{\mathbb{Q}}^{d}$, obey slightly different rules. Let $h \in \operatorname{Hom}_{\mathbb{Q}}\left(\mathbb{Q}^{d}, \mathbb{Q}\right)$ be a linear form such that $H$ is the closure of $h^{-1}(0)$ in $\mathbb{P}_{\mathbb{Q}}^{d}$. The line through 0 and $\mathbf{a} \in \mathbb{Q}^{d} \backslash\{0\}$ meets $\infty$ in a point that we denote a/0. For $q \in \mathbb{Q}$ let $\operatorname{sign}(q)$ be the usual signum function:

$$
\operatorname{sign}(q)= \begin{cases}-1 & \text { if } q<0 \\ 0 & \text { if } q=0 \\ 1 & \text { if } q>0\end{cases}
$$

Lemma 2.6. Let $B=\left\{\mathbf{b}_{1}, \ldots, \mathbf{b}_{n}\right\} \subseteq U_{H}$ with $B \cap \infty=\left\{\mathbf{b}_{m+1}, \ldots, \mathbf{b}_{n}\right\}$ and pick $\left\{\mathbf{b}_{m+1}^{\prime}, \ldots, \mathbf{b}_{n}^{\prime}\right\} \subseteq \mathbb{Q}^{d} \cap U_{H}$ such that $\mathbf{b}_{j}=\mathbf{b}_{j}^{\prime} / 0$ for $m<j \leq n$. If $\mathbf{b} \in$ $\operatorname{conv}_{H}(B) \cap \mathbb{Q}^{d}$ then in coordinates of $\mathbb{Q}^{d}$ there is an equation $\mathbf{b}=\sum_{j=1}^{m} \varepsilon_{j} \mathbf{b}_{j}+$ $\sum_{j=m+1}^{n} \varepsilon_{j}^{\prime} \mathbf{b}_{j}^{\prime}$ where $\sum_{j=1}^{m} \varepsilon_{j}=1, \operatorname{sign}\left(\varepsilon_{j} h\left(\mathbf{b}_{j}\right)\right)=\operatorname{sign}(h(\mathbf{b}))$ for all $j$ with $\varepsilon_{j} \neq 0$, and $\operatorname{sign}\left(\varepsilon_{j}^{\prime} h\left(\mathbf{b}_{j}^{\prime}\right)\right)=\operatorname{sign}(h(\mathbf{b}))$ for all $j$ with $\varepsilon_{j}^{\prime} \neq 0$.

Proof. Let $B_{+}$(resp. $B_{-}$) be the subsets of $B \backslash \infty$ on which $h$ evaluates positively (resp. negatively), and put $B_{\infty}=B \cap \infty, B^{\prime}=\left\{\mathbf{b}_{m+1}^{\prime}, \ldots, \mathbf{b}_{n}^{\prime}\right\}$. A general element $\mathbf{b} \in \operatorname{conv}_{H}(B)$ is the convex combination of three points: $\mathbf{b}_{+} \in \operatorname{conv}_{H}\left(B_{+}\right), \mathbf{b}_{-} \in$ $\operatorname{conv}_{H}\left(B_{-}\right)$, and $\mathbf{b}_{\infty} \in \operatorname{conv}_{H}\left(B_{\infty}\right)$.

Clearly, $\mathbf{b}_{+}=\sum_{\mathbf{b}_{j} \in B_{+}} \varepsilon_{j} \mathbf{b}_{j}$ where $\varepsilon_{j} \geq 0$ and $\sum_{\mathbf{b}_{j} \in B_{+}} \varepsilon_{j}=1$. A similar statement holds for $\mathbf{b}_{-}$. Now $\operatorname{conv}_{H}\left(\mathbf{b}_{+}, \mathbf{b}_{-}\right) \cap \mathbb{Q}^{d}$ is the union of rays $\left\{\lambda_{+} \mathbf{b}_{+}+\lambda_{-} \mathbf{b}_{-} \mid\right.$ $\left.\lambda_{+}+\lambda_{-}=1, \lambda_{+} \lambda_{-} \leq 0\right\}$. Thus, if $\mathbf{b}_{0} \in \operatorname{conv}_{H}\left(\mathbf{b}_{+}, \mathbf{b}_{-}\right)$then $\operatorname{sign}\left(\lambda_{+} h\left(\mathbf{b}_{+}\right)\right)=$ $\operatorname{sign}\left(\mathbf{b}_{0}\right)=\operatorname{sign}\left(\lambda_{-} h\left(\mathbf{b}_{-}\right)\right)$.

It suffices to show the lemma if $h\left(B^{\prime}\right)>0$. Then $\mathbf{b}_{\infty}$ is of the form $\mathbf{b}^{\prime} / 0$ with $\mathbf{b}^{\prime} \in \operatorname{conv}_{H}\left(B^{\prime}\right)$, and points of $\operatorname{conv}_{H}\left(B^{\prime}\right)$ are of the form $\sum_{\mathbf{b}_{j}^{\prime} \in B^{\prime}} \varepsilon_{j} \mathbf{b}_{j}^{\prime}$ with $\sum_{\mathbf{b}_{j}^{\prime} \in B^{\prime}} \varepsilon_{j}=1$ and all $\varepsilon_{j} \geq 0$. We are thus reduced to considering $\operatorname{conv}_{H}\left(\left\{\mathbf{b}_{0}, \mathbf{b}_{\infty}\right\}\right)$ with $\mathbf{b}_{\infty}=\mathbf{b}^{\prime} / 0$ and $h\left(\mathbf{b}^{\prime}\right)>0$. If $h\left(\mathbf{b}_{0}\right)>0$ then $\operatorname{conv}_{H}\left(\left\{\mathbf{b}_{0}, \mathbf{b}_{\infty}\right\}\right)$ is the ray $\left\{\mathbf{b}_{0}+\lambda^{\prime} \mathbf{b}^{\prime} \mid \lambda^{\prime} \geq 0\right\}$, while if $h\left(\mathbf{b}_{0}\right)<0$ then it is the ray $\left\{\mathbf{b}_{0}+\lambda^{\prime} \mathbf{b}^{\prime} \mid \lambda \leq 0\right\}$. The 
condition on $\lambda^{\prime}$ can, if $\lambda^{\prime} \neq 0$, be packaged as $\operatorname{sign}\left(\lambda^{\prime} h\left(\mathbf{b}^{\prime}\right)\right)=\operatorname{sign}\left(h\left(\mathbf{b}_{0}\right)\right)$. The lemma follows.

We view the columns $\mathbf{a}_{1}, \ldots, \mathbf{a}_{n} \in \mathbb{Z}^{d}$ of $A$ as points in $\mathbb{Q}^{d}=\mathbb{P}_{\mathbb{Q}}^{d} \backslash \infty$. By assumption, $\mathbb{N} A$ is positive and hence $h \in \operatorname{Hom}_{\mathbb{Q}}\left(\mathbb{Q}^{d}, \mathbb{Q}\right)$ can be chosen such that $h\left(\mathbf{a}_{j}\right)>0$ for all $j$. For any $\lambda \in \mathbb{Q}$, set $H_{\lambda}:=h^{-1}(-\lambda)$ and $U_{\lambda}:=\mathbb{P}_{\mathbb{Q}}^{d} \backslash H_{\lambda}$.

Definition 2.7. Choose $h \in \operatorname{Hom}_{\mathbb{Q}}\left(\mathbb{Q}^{d}, \mathbb{Q}\right)$ such that $h\left(\mathbf{a}_{j}\right)>0$ for all $j$, and let $\varepsilon$ be such that $0<\varepsilon<\left|h\left(\mathbf{a}_{j}\right) / L_{\partial_{j}}\right|$ whenever $L_{\partial_{j}} \neq 0$. We set $\mathbf{a}_{j}^{L}:=\mathbf{a}_{j} / L_{\partial_{j}}$ and call $\Delta_{A}^{L}:=\operatorname{conv}_{H_{\varepsilon}}\left(\left\{0, \mathbf{a}_{1}^{L}, \ldots, \mathbf{a}_{n}^{L}\right\}\right) \subseteq \mathbb{P}_{\mathbb{Q}}^{d}$ the $(A, L)$-polyhedron.

Let the $(A, L)$-umbrella be the set $\Phi_{A}^{L}$ of faces of $\Delta_{A}^{L}$ which do not contain 0 . In particular, $\Phi_{A}^{L}$ contains the empty face. Whenever it suits us, we identify $\tau \in \Phi_{A}^{L}$ with $\left\{j \mid \mathbf{a}_{j}^{L} \in \tau\right\}$, or with $\left\{\mathbf{a}_{j} \mid \mathbf{a}_{j}^{L} \in \tau\right\}$, or with the corresponding submatrix of $A$. By $\Phi_{A}^{L, k} \subseteq \Phi_{A}^{L}$, we denote the subset of faces of dimension $k$.

By $\Gamma_{A}^{L}:=\bigcup \Phi_{A}^{L}\left(\operatorname{resp} . \Gamma_{A}^{L, k}:=\bigcup \Phi_{A}^{L, k}\right)$, we denote the underlying point set of $\Phi_{A}^{L}\left(\operatorname{resp} . \Phi_{A}^{L, k}\right)$. Note that $\Gamma_{A}^{L}$ is a piecewise linear manifold with boundary, homeomorphic to the $(d-1)$-disk. If $\mathbf{a} \in \Delta_{A}^{L} \backslash\{0\}$ then the line through 0 and $\mathbf{a}$ meets $\Gamma_{A}^{L}$ in $\Gamma_{A}^{L}(\mathbf{a})$.

The matrix $A$ is called $L$-homogeneous if all $\mathbf{a}_{j}^{L}$ lie on a common hyperplane of $\mathbb{P}_{\mathbb{Q}}^{d}$. Every $A$ is $\mathbf{0}$-homogeneous and we call $\Phi_{A}^{0}$ the $A$-umbrella. If $A$ is $L$ -

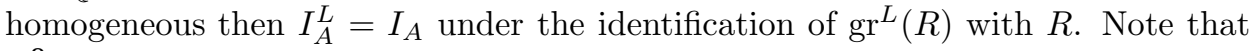
$\Phi_{A}^{0}$ can be identified with the lattice of nonempty faces of the polyhedral cone $\mathbb{Q}_{+} A$ via the face lattice of a cross-section $\mathbb{Q}_{+} A \cap h^{-1}(1)$.

Figure 2. More $(A, L)$-umbrellas. (Shaded $\Delta_{A}^{L}$ with fat boundary $\Phi_{A}^{L}$.)
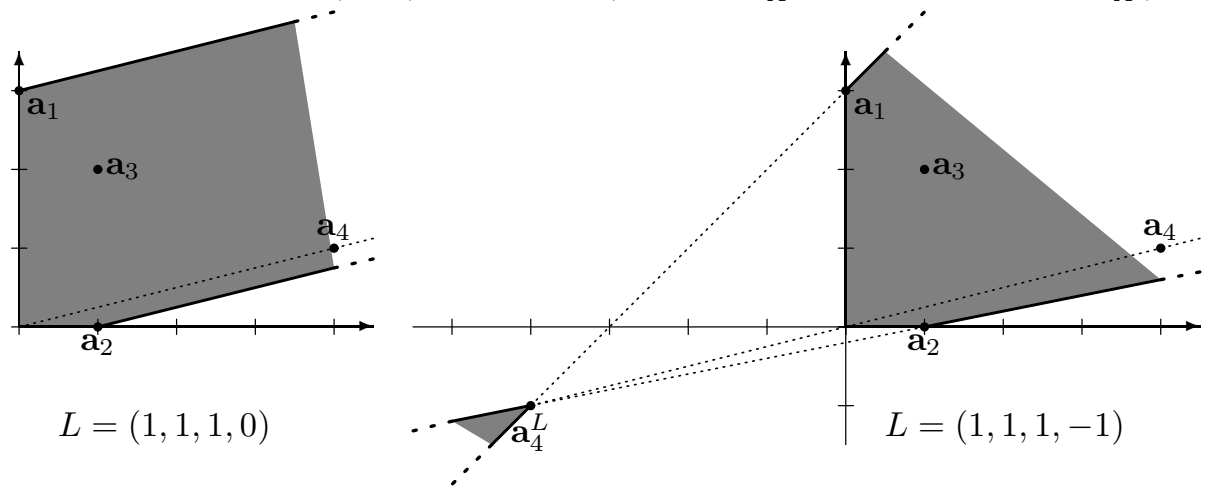

Figure 2 shows the intersection with $\mathbb{Q}^{d}$ of the $(A, L)$-umbrella for $L=(1,1,1, t)$ and $t \leq 0$ in the example from the introduction, $A=\left(\begin{array}{llll}0 & 1 & 1 & 4 \\ 3 & 0 & 2 & 1\end{array}\right)$.

2.4. Monomials in the graded toric ideal. The following result generalizes Lemma 3.1 and 3.2 in [Ado94.

Lemma 2.8. If $\mathbf{a}_{k_{1}}^{L}, \ldots, \mathbf{a}_{k_{m}}^{L} \in \Gamma_{A}^{L}$ do not lie in a common $\tau \in \Phi_{A}^{L, d-1}$ then $\partial_{k_{1}} \cdots \partial_{k_{m}} \in \sqrt{I_{A}^{L}}$ 
Proof. We write $v_{j}$ for $L_{\partial_{j}}$.

Assume first that $v_{k_{1}} \neq 0$ and $\Gamma_{A}^{L}\left(\mathbf{a}_{k_{1}}\right)=\mathbf{a}_{k_{1}} / 0 \in \infty$. In particular, $v_{k_{1}}>0$ by Definition 2.7. The polyhedron $\Delta_{A}^{L} \cap \infty$ is the convex hull of the points $\mathbf{a}_{i}^{L}=\mathbf{a}_{i} / 0$ with $v_{i}=0$, and of the intersection points $\left(\mathbf{a}_{i}^{L}-\mathbf{a}_{j}^{L}\right) / 0$ with $\infty$ of line segments from $\mathbf{a}_{i}^{L}$ to $\mathbf{a}_{j}^{L}$ with $v_{i}>0>v_{j}$. Therefore

$$
\mathbf{a}_{k_{1}}^{L}=\sum_{v_{i}=0} \eta_{i} \mathbf{a}_{i}+\sum_{v_{i}>0>v_{j}} \eta_{i, j}\left(\mathbf{a}_{i}^{L}-\mathbf{a}_{j}^{L}\right)
$$

for some $0 \leq \eta_{i}, \eta_{i, j} \in \mathbb{Q}$. This equality gives rise to an element

$$
\square=\partial_{k_{1}}^{s / v_{k}}-\prod_{v_{i}=0} \partial_{i}^{s \eta_{i}} \prod_{v_{i}>0>v_{j}} \partial_{i}^{s \eta_{i, j} / v_{i}} \partial_{j}^{-s \eta_{i, j} / v_{j}} \in I_{A}
$$

where $s \in \mathbb{N}$ is chosen to clear all denominators. The $L$-degree of the left monomial is positive while that of the right one is zero. Thus $\sigma^{L}(\square)=\partial_{k_{1}}^{s / v_{k}} \in \operatorname{gr}^{L}\left(I_{A}\right)$ and the claim follows in this case.

We now keep the assumption $v_{k_{1}} \neq 0$ but assume that $\Gamma_{A}^{L}\left(\mathbf{a}_{k_{1}}\right) \notin \infty$. By hypothesis there is $\mathbf{a} \in \operatorname{conv}\left(\mathbf{a}_{k_{1}}^{L}, \ldots, \mathbf{a}_{k_{m}}^{L}\right)$ in the interior of $\Delta_{A}^{L}$. By continuity of the function $\mathbf{a} \mapsto \Gamma_{A}^{L}(\mathbf{a})$, choosing a sufficiently close to $\mathbf{a}_{k_{1}}^{L}$ implies that $\Gamma_{A}^{L}(\mathbf{a}) \notin \infty$. As a lies in the interior of $\Delta_{A}^{L}$ and since $\Gamma_{A}^{L}(\mathbf{a}) \notin \infty$, there is $0 \neq t \in \mathbb{Q}$ with $\Gamma_{A}^{L}(\mathbf{a})=t \mathbf{a} \in \Gamma_{A}^{L}$ and hence either $\operatorname{sign}(t h(\mathbf{a}))=1$ and $t>1$, or $\operatorname{sign}(t h(\mathbf{a}))=-1$ and $0<t<1$. In either case, $t \operatorname{sign}(t h(\mathbf{a}))>\operatorname{sign}(t h(\mathbf{a}))$. Using Lemma 2.6, we can write

$$
\mathbf{a}=\sum_{v_{k_{j}} \neq 0} \varepsilon_{k_{j}} \mathbf{a}_{k_{j}}^{L}+\sum_{v_{k_{j}}=0} \varepsilon_{k_{j}} \mathbf{a}_{k_{j}}
$$

for some $\varepsilon_{k_{j}} \in \mathbb{Q}$ with $\sum_{v_{k_{j}} \neq 0} \varepsilon_{k_{j}}=1, \operatorname{sign}\left(\varepsilon_{k_{j}} / v_{k_{j}}\right)=\operatorname{sign}(h(\mathbf{a}))$ if $\varepsilon_{k_{j}} v_{k_{j}} \neq 0$, and $\operatorname{sign}\left(\varepsilon_{k_{j}}\right)=\operatorname{sign}(h(\mathbf{a}))$ if $\varepsilon_{k_{j}} \neq 0=v_{k_{j}}$. Again by Lemma 2.6 and Definition 2.7. we can write

$$
t \mathbf{a}=\sum_{v_{i} \neq 0} \eta_{i} \mathbf{a}_{i}^{L}+\sum_{v_{i}=0} \eta_{i} \mathbf{a}_{i}
$$

for some $\eta_{i} \in \mathbb{Q}$ where $\sum_{v_{i} \neq 0} \eta_{i}=1, \operatorname{sign}\left(\eta_{i} / v_{i}\right)=\operatorname{sign}(t h(\mathbf{a}))$ if $\eta_{i} v_{i} \neq 0$, and $\operatorname{sign}\left(\eta_{i}\right)=\operatorname{sign}(\operatorname{th}(\mathbf{a}))$ if $\eta_{i} \neq 0=v_{i}$. Combining equations (2.4.1) and (2.4.2) we find an element

$$
\square=\prod_{v_{k_{j}} \neq 0} \partial_{k_{j}}^{s\left|t \varepsilon_{k_{j}} / v_{k_{j}}\right|} \prod_{v_{k_{j}}=0} \partial_{k_{j}}^{s\left|t \varepsilon_{k_{j}}\right|}-\prod_{v_{i} \neq 0} \partial_{i}^{s\left|\eta_{i} / v_{i}\right|} \prod_{v_{i}=0} \partial_{i}^{s\left|\eta_{i}\right|} \in I_{A}
$$

where $s \in \mathbb{N}$ chosen to clear all denominators. From

$$
\begin{array}{r}
\operatorname{deg}^{L}\left(\prod_{v_{k_{j}} \neq 0} \partial_{k_{j}}^{s\left|t \varepsilon_{k_{j}} / v_{k_{j}}\right|} \prod_{v_{k_{j}}=0} \partial_{k_{j}}^{s\left|t \varepsilon_{k_{j}}\right|}\right)=s|t| \sum_{v_{k_{j}} \neq 0} \varepsilon_{k_{j}} \operatorname{sign}\left(\varepsilon_{k_{j}} / v_{k_{j}}\right) \\
=s t \sum_{v_{k_{j}} \neq 0} \varepsilon_{k_{j}} \operatorname{sign}(\operatorname{th}(\mathbf{a}))=s t \operatorname{sign}(\operatorname{th}(\mathbf{a}))>s \operatorname{sign}(\operatorname{th}(\mathbf{a})) \\
=s \sum_{v_{i} \neq 0} \operatorname{sign}\left(\eta_{i} / v_{i}\right) \eta_{i}=s \sum_{v_{i} \neq 0}\left|\eta_{i} / v_{i}\right| v_{i}=\operatorname{deg}^{L}\left(\prod_{v_{i} \neq 0} \partial_{i}^{s\left|\eta_{i} / v_{i}\right|} \prod_{v_{i}=0} \partial_{i}^{s\left|\eta_{i}\right|}\right),
\end{array}
$$

we conclude that the $L$-leading term of $\square$ is the left of the two monomials in (2.4.3). The claim follows in the case where at least one $v_{k_{j}}$ is nonzero. 
Suppose finally that $v_{k_{j}}=0$ and hence $\mathbf{a}_{k_{j}}^{L} \in \infty$ for all $j=1, \ldots, m$. By assumption and Definition 2.7 we can pick an element $\mathbf{a}^{\prime} \in \operatorname{conv}\left(\mathbf{a}_{k_{1}}^{L}, \ldots, \mathbf{a}_{k_{m}}^{L}\right) \backslash$ $\partial \Delta_{A}^{L} \subseteq \operatorname{conv}\left(\mathbf{a}_{k_{1}}^{L}, \ldots, \mathbf{a}_{k_{m}}^{L}\right) \backslash \Gamma_{A}^{L}$. Then $\mathbf{a}^{\prime}=\mathbf{a} / 0$ with $\mathbf{a} \in \operatorname{conv}\left(\mathbf{a}_{k_{1}}, \ldots, \mathbf{a}_{k_{m}}\right)$. It follows that there is an equation of type (2.4.1) with conditions as indicated there. By construction, $\Gamma_{A}^{L}\left(\mathbf{a}^{\prime}\right) \neq \mathbf{a}^{\prime} \in \infty$ and so $\Gamma_{A}^{L}\left(\mathbf{a}^{\prime}\right) \notin \infty$. Hence, there is a positive $t \in \mathbb{Q}$ with $\Gamma_{A}^{L}\left(\mathbf{a}^{\prime}\right)=t \mathbf{a}$ and so there is an equation of type (2.4.2) with conditions as indicated there. As $t \operatorname{sign}(t h(\mathbf{a}))=-t>0>-1=\operatorname{sign}(t h(\mathbf{a}))$ we get an equation of type (2.4.4), and the claim follows as in the previous case.

\subsection{Homogeneity in the graded toric ideal.}

Definition 2.9. Let $\tau$ be a set of columns of $A$. For $\mathbf{u} \in \mathbb{Z}^{n}$, we write $\operatorname{supp}(\mathbf{u}) \subseteq \tau$ if $\mathbf{u}_{i} \neq 0$ implies $\mathbf{a}_{i} \in \tau$. For $f \in R$, we write $\operatorname{supp}(f) \subseteq \tau$ if $\operatorname{supp}(\mathbf{u}) \subseteq \tau$ for all monomials $\partial^{\mathbf{u}}$ of $f$.

Lemma 2.10. Let $\tau \in \Phi_{A}^{L}$ and pick $\mathbf{u} \in \mathbb{Z}^{n}$ such that $A \cdot \mathbf{u}=0$.

(1) If $\operatorname{supp}\left(\square_{\mathbf{u}}\right) \subseteq \tau$ then $\sigma^{L}\left(\square_{\mathbf{u}}\right)=\square_{\mathbf{u}}$. In particular, the toric ideal $I_{\tau}$ is L-homogeneous.

(2) If $\operatorname{supp}\left(\partial^{\mathbf{u}_{ \pm}}\right) \subseteq \tau$ and $\operatorname{supp}\left(\partial^{\mathbf{u}_{\mp}}\right) \nsubseteq \tau$ then $\sigma^{L}\left(\square_{\mathbf{u}}\right)=\mp \partial^{\mathbf{u}_{\mp}}$.

Proof. We write $v_{j}$ for $L_{\partial_{j}}$.

Consider first the case where the facet $\tau \in \Phi_{A}^{L, d-1}$ lies entirely in $\infty$. Then $\mathbf{a}_{i}^{L} \in \tau$ implies $v_{i}=0$ and hence $\operatorname{supp}\left(\square_{\mathbf{u}}\right) \subseteq \tau$ implies that $\square_{\mathbf{u}}$ is $L$-homogeneous of degree zero. Suppose $\operatorname{supp}\left(\mathbf{u}_{+}\right) \subseteq \tau$ but $\operatorname{supp}\left(\mathbf{u}_{-}\right) \not \subset$. As $\tau \subseteq \infty$ is a facet of $\Delta_{A}^{L}$ and by Definition 2.7, the interior of $\Delta_{A}^{L}$ meets neither $\infty$ nor $H_{\varepsilon}$. Hence $\Delta_{A}^{L}$ is

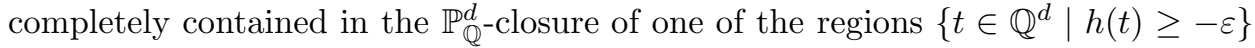
and $\left\{t \in \mathbb{Q}^{d} \mid h(t) \leq-\varepsilon\right\}$. Since $0 \in \Delta_{A}^{L}$, it must be the former. Moreover, by definition of $\varepsilon, h\left(\mathbf{a}_{j}^{L}\right) \notin[-\varepsilon, 0]$ for all $v_{j} \neq 0$, whence $v_{j} \geq 0$ in all cases. Thus $\operatorname{deg}^{L}\left(\partial^{\mathbf{u}_{+}}\right)=0$ and $\operatorname{deg}^{L}\left(\partial^{\mathbf{u}_{-}}\right)>0$, hence $\sigma^{L}\left(\square_{\mathbf{u}}\right)=-\partial^{\mathbf{u}_{-}}$. The case $\operatorname{supp}\left(\mathbf{u}_{-}\right) \subseteq \tau \nsupseteq \operatorname{supp}\left(\mathbf{u}_{+}\right)$is similar.

Now let $\tau \in \Phi_{A}^{L}$ be not contained in $\infty$, or let $\tau \in \Phi_{A}^{L} \backslash \Phi_{A}^{L, d-1}$ be a nonfacet face contained in $\infty$. Then there is a linear form $h_{\tau} \in \operatorname{Hom}_{\mathbb{Q}}\left(\mathbb{Q}^{d}, \mathbb{Q}\right)$ such that the closure of $h_{\tau}^{-1}(1)$ in $\mathbb{P}_{\mathbb{Q}}^{d}$ meets $\Delta_{A}^{L}$ precisely in $\tau$, and for which $\operatorname{sign}\left(v_{i}\right) h_{\tau}\left(\mathbf{a}_{i}^{L}\right) \leq \operatorname{sign}\left(v_{i}\right)$ whenever $v_{i} \neq 0$.

For all $\mathbf{a}_{i}^{L} \in \tau$ with $v_{i}=0$ the line through 0 and $\mathbf{a}_{i}$ meets $\tau$ in $\mathbf{a}_{i}^{L} \in \infty$. This means that $\mathbf{a}_{i}$ is parallel to $h_{\tau}^{-1}(1)$ and so $h_{\tau}\left(\mathbf{a}_{i}\right)=0$. Thus, $A \cdot \mathbf{u}=0$ implies that

$$
0=h_{\tau}(A \cdot \mathbf{u})=h_{\tau}\left(\sum_{i=1}^{n} u_{i} \mathbf{a}_{i}\right)=\sum_{u_{i}>0 \neq v_{i}} u_{i} v_{i} h_{\tau}\left(\mathbf{a}_{i}^{L}\right)-\sum_{u_{i}<0 \neq v_{i}}\left(-u_{i}\right) v_{i} h_{\tau}\left(\mathbf{a}_{i}^{L}\right) .
$$

If $\operatorname{supp}\left(\square_{\mathbf{u}}\right) \subseteq \tau$ and $u_{i} v_{i} \neq 0$ then $h_{\tau}\left(\mathbf{a}_{i}^{L}\right)=1$ and hence $\operatorname{deg}^{L}\left(\partial^{\mathbf{u}_{+}}\right)=\operatorname{deg}^{L}\left(\partial^{\mathbf{u}_{-}}\right)$.

Now suppose $\operatorname{supp}\left(\partial^{\mathbf{u}_{+}}\right) \subseteq \tau \nsupseteq \operatorname{supp}\left(\partial^{\mathbf{u}_{-}}\right)$. Hence $h_{\tau}\left(\mathbf{a}_{i}^{L}\right)=1$ if $u_{i}>0 \neq v_{i}$, $\operatorname{sign}\left(v_{i}\right) h_{\tau}\left(\mathbf{a}_{i}^{L}\right) \leq \operatorname{sign}\left(v_{i}\right)$ if $u_{i}<0 \neq v_{i}$, and $\operatorname{sign}\left(v_{i}\right) h_{\tau}\left(\mathbf{a}_{i}^{L}\right)<\operatorname{sign}\left(v_{i}\right)$ for at least one $u_{i}<0$. Thus,

$$
\begin{aligned}
\operatorname{deg}^{L}\left(\partial^{\mathbf{u}_{+}}\right)=\sum_{u_{i}>0} v_{i} u_{i} & =\sum_{u_{i}>0 \neq v_{i}} v_{i} u_{i} h_{\tau}\left(\mathbf{a}_{i}^{L}\right) \\
& =\sum_{u_{i}<0 \neq v_{i}} v_{i}\left(-u_{i}\right) h_{\tau}\left(\mathbf{a}_{i}^{L}\right)<\sum_{u_{i}<0 \neq v_{i}} v_{i}\left(-u_{i}\right)=\operatorname{deg}^{L}\left(\partial^{\mathbf{u}_{-}}\right) .
\end{aligned}
$$

The case $\operatorname{supp}\left(\mathbf{u}_{-}\right) \subseteq \tau \nsupseteq \operatorname{supp}\left(\mathbf{u}_{+}\right)$is similar. 
2.6. Minimal associated prime ideals. We now identify the components of the $L$-graded toric ring $S_{A}^{L}$.

Definition 2.11. For $\mathbf{u} \in \mathbb{Z}^{n}$ let

$$
\tau_{\mathbf{u}}^{L}=\bigcap_{\operatorname{supp}(\mathbf{u}) \subseteq \tau \in \Phi_{A}^{L}} \tau
$$

be the smallest element of $\Phi_{A}^{L}$ containing $\operatorname{supp}(\mathbf{u})$ and put $\tau_{\mathbf{u}}^{L}=A$ if there is none. Note that $\left[\partial^{\mathbf{u}} \in J_{\tau}\right] \Rightarrow\left[\tau_{\mathbf{u}}^{L} \nsubseteq \tau\right]$ for $\tau \subseteq A$ and $\mathbf{u} \in \mathbb{N}^{n}$.

Recall that $I_{A}, I_{A}^{\tau}$, and $J_{\tau}$ are $\mathbb{Z}^{d}$-graded prime ideals.

Lemma 2.12. Let $\tilde{I}_{A}^{L} \subseteq R$ be generated by all elements of the following types:

(1) $\partial_{k_{1}} \cdots \partial_{k_{m}}$ where $\mathbf{a}_{k_{1}}^{L}, \ldots, \mathbf{a}_{k_{m}}^{L}$ do not lie in a common facet $\tau \in \Phi_{A}^{L, d-1}$;

(2) $\square_{\mathbf{u}}$ where $\mathbf{u} \in \mathbb{Z}^{n}, A \cdot \mathbf{u}=0$, and $\tau_{\mathbf{u}}^{L} \neq A$.

Then $\tilde{I}_{A}^{L}=\bigcap_{\tau \in \Phi_{A}^{L, d-1}} I_{A}^{\tau}$.

Proof. Let $I=\bigcap_{\tau \in \Phi_{A}^{L, d-1}} I_{A}^{\tau}$. We show first that $\tilde{I}_{A}^{L} \subseteq I$. Let $I_{0}$ denote the ideal of $R$ generated by the elements from (11). Then clearly $I_{0} \subseteq J_{\tau} \subseteq I_{A}^{\tau}$ for any $\tau \in \Phi_{A}^{L, d-1}$. Pick $\square_{\mathbf{u}}$ as in (2). By part (11) of Lemma 2.10, $\sigma^{L}\left(\square_{\mathbf{u}}\right)=\square_{\mathbf{u}}$. Let $\tau \in \Phi_{A}^{L, d-1}$ and suppose $\square_{\mathbf{u}} \notin J_{\tau}$. Then (without loss of generality) $\operatorname{supp}\left(\mathbf{u}_{+}\right) \subseteq \tau$. By part (2) of Lemma 2.10, since $\sigma^{L}\left(\square_{\mathbf{u}}\right)=\square_{\mathbf{u}}, \operatorname{supp}\left(\mathbf{u}_{-}\right) \subseteq \tau$ as well. Hence $\square_{\mathbf{u}} \in I_{\tau} \subseteq I_{A}^{\tau}$ and so $\tilde{I}_{A}^{L} \subseteq I$.

For the converse inclusion suppose now $m \in I$. We write $m=m_{0}+\sum_{\tau \in \Phi_{A}^{L}} m_{\tau}$ where $m_{0} \in I_{0}$, and

$$
m_{\tau}=\sum_{\tau_{\mathbf{u}}^{L}=\tau} c_{\mathbf{u}} \partial^{\mathbf{u}} \in \mathbb{C}\left[\partial_{\tau}\right]
$$

collects the monomials in $m$ minimally supported in $\tau$. Since $I_{0} \subseteq \tilde{I}_{A}^{L} \subseteq I$ we may assume that $m_{0}=0$. Now pick any $\tau \in \Phi_{A}^{L}$. Then $m=\hat{m}_{\tau}+m_{\bar{\tau}}$ where $\hat{m}_{\tau}=\sum_{\tau^{\prime} \subseteq \tau} m_{\tau^{\prime}}$ are the terms in $m$ supported in $\tau$. Since $m_{\bar{\tau}} \in J_{\tau} \subseteq I_{A}^{\tau}$ and $m \in I \subseteq I_{A}^{\tau}, \hat{m}_{\tau}=m-m_{\bar{\tau}} \in I_{A}^{\tau} \cap \mathbb{C}\left[\partial_{\tau}\right]=I_{\tau} \subseteq \tilde{I}_{A}^{L}$ in view of (2). Since every $\hat{m}_{\tau}$ is in $\tilde{I}_{A}^{L}$, so is every $m_{\tau}$, and hence $m \in \tilde{I}_{A}^{L}$ as well.

Lemma 2.13. The radical ideal $\tilde{I}_{A}^{L}$ from Lemma 2.12 equals $\sqrt{I_{A}^{L}}$.

Proof. By Lemma 2.8, the elements in 2.12, (11) are in $\sqrt{I_{A}^{L}}$. By part (1) of Lemma 2.10, all elements from 2.12(2) are in $I_{A}^{L}$. Hence $\tilde{I}_{A}^{L} \subseteq \sqrt{I_{A}^{L}}$.

By Lemmas 2.4 and 2.12, it suffices to show conversely that, for any $\mathbf{u} \in \mathbb{Z}^{n}$ with $A \cdot \mathbf{u}=0, \sigma^{L}\left(\square_{\mathbf{u}}\right) \in I_{A}^{\tau}$ for all $\tau \in \Phi_{A}^{L, d-1}$. Pick such $\mathbf{u}$ and let $\tau \in \Phi_{A}^{L, d-1}$. If $\tau_{\mathbf{u}}^{L} \subseteq \tau$ then by part (10) of Lemma 2.10, $\sigma^{L}\left(\square_{\mathbf{u}}\right)=\square_{\mathbf{u}} \in I_{\tau} \subseteq I_{A}^{\tau}$. If $\tau_{\mathbf{u}_{+}}^{L^{A}} \subseteq \tau$ but $\tau_{\mathbf{u}_{-}}^{L} \nsubseteq \tau$ then by part (2) of Lemma 2.10, $\sigma^{L}\left(\square_{\mathbf{u}}\right)=-\partial^{\mathbf{u}_{-}} \in J_{\tau} \subseteq I_{A}^{\tau}$. Similarly, $\sigma^{L}\left(\square_{\mathbf{u}}\right) \in I_{A}^{\tau}$ if $\tau_{\mathbf{u}_{-}}^{L} \subseteq \tau$ but $\tau_{\mathbf{u}_{+}}^{L} \nsubseteq \simeq \tau$. Finally, if $\tau_{\mathbf{u}_{+}}^{L} \nsubseteq \tau \rrbracket \tau_{\mathbf{u}_{-}}^{L}$ then $\sigma^{L}\left(\square_{\mathbf{u}}\right) \in J_{\tau} \subseteq I_{A}^{\tau}$.

The following consequence of Lemmas 2.3, 2.12, and 2.13 generalizes Ado94, Lem. 3.2]. 
Theorem 2.14. The set of $\mathbb{Z}^{d}$-graded prime ideals of $R$ containing $I_{A}^{L}$ equals $\left\{I_{A}^{\tau} \mid\right.$ $\left.\tau \in \Phi_{A}^{L}\right\}$ and hence the $(A, L)$-umbrella encodes the geometry of $S_{A}^{L}$ :

$$
\operatorname{Spec}\left(S_{A}^{L}\right)=\operatorname{Var}\left(I_{A}^{L}\right)=\bigcup_{\tau \in \Phi_{A}^{L, d-1}} \bar{O}_{A}^{\tau}=\bigsqcup_{\tau \in \Phi_{A}^{L}} O_{A}^{\tau} .
$$

Adjacencies of orbit strata correspond to inclusions in the $(A, L)$-umbrella:

$$
\left[O_{A}^{\tau^{\prime}} \subseteq \bar{O}_{A}^{\tau}\right] \Leftrightarrow\left[\tau^{\prime} \subseteq \tau\right]
$$

In particular, Theorem 2.14 identifies the $\mathbb{Z}^{d}$-graded prime ideals containing $I_{A}=I_{A}^{0}$ with the elements of $\Phi_{A}^{0}$ from Definition 2.7

Remark 2.15. Geometrically, $\operatorname{Spec}\left(S_{A}^{L}\right)$ reflects certain asymptotics of $\operatorname{Spec}\left(S_{A}\right)$. We make this precise in the case where $L_{\partial_{j}}>0$ for all $j$. We may assume that the components of $L$ are coprime positive integers. Then $X=\mathbb{C}^{n}$ can be considered as the smooth chart defined by $\partial_{0} \neq 0$ in the weighted projective space $\mathbb{P}_{(1, L)}^{n}$ where $(1, L):=\left(1, L_{\partial_{1}}, \ldots, L_{\partial_{n}}\right)$, and $\operatorname{Spec}\left(S_{A}\right) \subseteq X$. The closure $Z$ of $\operatorname{Spec}\left(S_{A}\right)$ in $\mathbb{P}_{(1, L)}^{n}$ is defined by the $L$-homogenization $I_{A}^{L}\left(\partial_{0}\right)$ of $I_{A}$ with respect to $\partial_{0}$. The ideal of the intersection of $Z$ with the weighted projective space $\mathbb{P}_{L}^{n-1}=\operatorname{Var}\left(\partial_{0}\right) \subseteq \mathbb{P}_{(1, L)}^{n}$ is $I_{A}^{L}\left(\partial_{0}\right)_{\mid \partial_{0}=0}=I_{A}^{L}$. Thus $Z \cap \mathbb{P}_{L}^{n-1}=\operatorname{Proj}\left(S_{A}^{L}\right) \subseteq \mathbb{P}_{L}^{n-1}$.

2.7. Index formula for multiplicities. By Theorem 2.14, there is a composition chain of $\mathbb{Z}^{d}$-graded $R$-modules

$$
0=N_{0} \subsetneq N_{1} \subsetneq \cdots \subsetneq N_{l-1} \subsetneq N_{l}=S_{A}^{L}
$$

with $N_{i} / N_{i-1} \cong S_{\tau_{i}}\left(-\mathbf{u}_{i}\right)$ for some $\tau_{i} \in \Phi_{A}^{L}$ and $\mathbf{u}_{i} \in \mathbb{Z}^{d}$. As $\mathbb{Z}^{d}$-graded vector spaces $S_{A}^{L}=S_{A}$ and the $\mathbb{Z}^{d}$-graded Hilbert function of both rings has values in $\{0,1\}$. Thus, the composition chain (2.7.1) induces a partition of $\mathbb{Z}^{d}$-degrees

$$
\operatorname{deg}\left(S_{A}^{L}\right)=\operatorname{deg}\left(S_{A}\right)=\mathbb{N} A=\bigsqcup_{i=1}^{l}\left(\mathbf{u}_{i}+\mathbb{N} \tau_{i}\right)=\bigsqcup_{i=1}^{l} \operatorname{deg}\left(S_{\tau_{i}}\left(-\mathbf{u}_{i}\right)\right)
$$

Definition 2.16. If $\tau \in \Phi_{A}^{L, d-1}$ is a facet then the number $\nu_{A}^{L, \tau}$ of indices $i$ in the chain (2.7.1), and hence in the partition (2.7.2), with $\tau_{i}=\tau$ is the multiplicity of $S_{A}^{L}$ along $\bar{O}_{A}^{\tau}$. Note that $\nu_{A}^{L, \tau}$ is the length of the localization of $S_{A}^{L}$ at $I_{A}^{\tau}$ and hence independent of the particular composition chain.

Proposition 2.17. For all $\tau \in \Phi_{A}^{L, d-1}, \nu_{A}^{L, \tau}=\left[\mathbb{Z}^{d}: \mathbb{Z} \tau\right]$. In particular, the degree of $I_{A}^{L}$ equals $\sum_{\tau \in \Phi_{A}^{L, d-1}} \nu_{A}^{L, \tau}=\sum_{\tau \in \Phi_{A}^{L, d-1}}\left[\mathbb{Z}^{d}: \mathbb{Z} \tau\right]$.

Proof. Fix $\tau \in \Phi_{A}^{L, d-1}$. For disjoint $\mathbf{u}+\mathbb{N} \tau$ and $\mathbf{v}+\mathbb{N} \tau, \mathbf{u}-\mathbf{v} \notin \mathbb{Z} \tau$ since $\mathbb{N} \tau$ contains a shifted copy of its normalization $\mathbb{Q}_{+} \tau \cap \mathbb{Z} \tau$. This means that $\nu_{A}^{L, \tau} \leq\left[\mathbb{Z}^{d}: \mathbb{Z} \tau\right]$ and it remains to show that $\bigsqcup_{\tau_{i}=\tau}\left(\mathbf{u}_{i}+\mathbb{N} \tau_{i}\right)$ meets every coset of $\mathbb{Z}^{d} / \mathbb{Z} \tau$. Pick $\mathbf{u} \in \mathbb{N} \tau$ outside $\mathbb{Q}_{+} \tau^{\prime}$ for all $\tau^{\prime} \in \Phi_{A}^{L}$ with $\tau^{\prime} \subsetneq \tau$. Since $\mathbb{N} A$ contains a shifted copy of its normalization $\mathbb{Q}_{+} A \cap \mathbb{Z}^{d}$, for $k \gg 0$

$$
k \mathbf{u}+\left(\mathbb{Q}_{+} \tau \cap \mathbb{Z}^{d}\right) \subseteq k \mathbf{u}+\left(\mathbb{Q}_{+} A \cap \mathbb{Z}^{d}\right) \subseteq \mathbb{N} A=\operatorname{deg}\left(S_{A}^{L}\right)=\bigsqcup_{i=1}^{l} \operatorname{deg}\left(S_{\tau_{i}}\left(-\mathbf{u}_{i}\right)\right)
$$


By Lemma 2.18 below, for $k \gg 0$

$$
k \mathbf{u}+\left(\mathbb{Q}_{+} \tau \cap \mathbb{Z}^{d}\right) \subseteq \bigsqcup_{\tau_{i}=\tau}\left(\mathbf{u}_{i}+\mathbb{N} \tau_{i}\right)
$$

and the left hand side meets every coset of $\mathbb{Z}^{d} / \mathbb{Z} \tau$. This yields $\nu_{A}^{L, \tau} \geq\left[\mathbb{Z}^{d}: \mathbb{Z} \tau\right]$.

Lemma 2.18. Let $\tau \in \Phi_{A}^{L, d-1}$ and suppose $\mathbf{u} \in \mathbb{N} \tau \backslash \mathbb{Q}_{+} \tau^{\prime}$ for all $\tau^{\prime} \in \Phi_{A}^{L}$ with $\tau^{\prime} \subsetneq \tau$. Fix $\mathbf{u}^{\prime} \in \mathbb{Z}^{d}$ and $\tau^{\prime} \in \Phi_{A}^{L}$ such that $\tau^{\prime} \neq \tau$. Then for $k \gg 0$

$$
\left(k \mathbf{u}+\mathbb{Q}_{+} \tau\right) \cap\left(\mathbf{u}^{\prime}+\mathbb{Q}_{+} \tau^{\prime}\right)=\emptyset .
$$

Proof. Suppose that $k \mathbf{u}+\mathbf{v}=\mathbf{u}^{\prime}+\mathbf{v}^{\prime}$ with $\mathbf{v} \in \mathbb{Q}_{+} \tau$ and $\mathbf{v}^{\prime} \in \mathbb{Q}_{+} \tau^{\prime}$. Pick a linear form $0 \neq h_{\tau, \tau^{\prime}} \in \operatorname{Hom}_{\mathbb{Q}}\left(\mathbb{Q}^{d}, \mathbb{Q}\right)$ with $h_{\tau, \tau^{\prime}}(\tau) \geq 0, h_{\tau, \tau^{\prime}}\left(\tau^{\prime}\right) \leq 0$. Then, by hypothesis, $h_{\tau, \tau^{\prime}}(\mathbf{u})>0$. But $k h_{\tau, \tau^{\prime}}(\mathbf{u})+h_{\tau, \tau^{\prime}}(\mathbf{v})=h\left(\mathbf{u}^{\prime}\right)+h_{\tau, \tau^{\prime}}\left(\mathbf{v}^{\prime}\right)$ implies that $k h_{\tau, \tau^{\prime}}(\mathbf{u}) \leq h\left(\mathbf{u}^{\prime}\right)$ which is impossible for $k \gg 0$.

2.8. Newton filtration and Cohen-Macaulayness. Until the end of this section, we fix a weight vector $L$ with $L_{\partial_{i}}>0$ for all $i$. Let $V=\bigoplus_{\mathbf{a} \in \mathbb{N} A} V_{\mathbf{a}}$ be a $\mathbb{Z}^{d}$-graded vector space. Then the Newton filtration $N=N_{A}^{L}$ on $V$ with respect to $\Delta_{A}^{L}$ is defined by

$$
N_{i} V=\bigoplus_{\mathbf{a} \in i \cdot \Delta_{A}^{L}} V_{\mathbf{a}} .
$$

Note that $\operatorname{gr}^{N}(V)=V$ as $\mathbb{Z}^{d}$-graded vector spaces. For $\tau \in \Phi_{A}^{L}$ or $\tau=A$, we denote $V(\tau)=\bigoplus_{\mathbf{a} \in \mathbb{Q}_{+} \tau} V_{\mathbf{a}}$. Generalizing [Kou76, Prop. 2.6], there is a complex

$$
0 \longrightarrow V_{d-1} \longrightarrow V_{d-2} \longrightarrow \cdots \longrightarrow V_{0} \longrightarrow 0
$$

where the $\mathbb{Z}^{d}$-graded vector space $V_{i}$ is the direct sum of all $V(\tau)$ for which $\tau \in \Phi_{A}^{L, i}$ is not contained in the boundary of $\Gamma_{A}^{L}$ (see Definition 2.7). The cohomology of this complex is concentrated in homological degree $d-1$ and equal to $V$.

Now assume that $V$ is a $\mathbb{Z}^{d}$-graded $\mathbb{C}$-algebra. Then $\operatorname{gr}^{N}(V)(\tau)=V(\tau)$ as $\mathbb{C}$ algebras for $\tau \in \Phi_{A}^{L}$. For $\tau, \tau^{\prime} \in \Phi_{A}^{L}$ (or $\tau=A$ ) with $\tau \supseteq \tau^{\prime}$, the maps

$$
\gamma_{\tau, \tau^{\prime}}: \operatorname{gr}^{N}(V)(\tau) \longrightarrow \operatorname{gr}^{N}(V)\left(\tau^{\prime}\right)
$$

in the complex (2.8.1) for $\operatorname{gr}^{N}(V)$ are natural projections of $\mathbb{C}$-algebras. In particular, (2.8.1) is a complex of $\mathrm{gr}^{N}(V)$-modules.

Lemma 2.19. For all $\tau \in \Phi_{A}^{L}$ and all $k>0, \mathbb{C}\left[\mathbb{N} \cdot k \cdot\left(\tau \cap \Phi_{A}^{L, 0}\right)\right] \subseteq S_{A}^{L}(\tau)$ is a module-finite ring extension for all $\tau \in \Phi_{A}^{L}$.

Proof. One can construct a $\mathbb{Z}^{d}$-graded composition chain of $S_{A}^{L}$ as in (2.7.1) such that the resulting partition (2.7.2) refines the partition $\mathbb{Q}_{+} A=\mathbb{Q}_{+} \tau \sqcup\left(\mathbb{Q}_{+} A \backslash \mathbb{Q}_{+} \tau\right)$, and hence $\operatorname{deg}\left(S_{\tau_{i}}\left(-\mathbf{u}_{i}\right)\right)$ meets $\mathbb{Q}_{+} \tau$ only if $\tau_{i} \subseteq \tau$. Namely, given any chain as in (2.7.1) we refine it at any index $i \in\{1, \ldots, l\}$ for which $\operatorname{deg}\left(S_{\tau_{i}}\left(-\mathbf{u}_{i}\right)\right)=\mathbf{u}_{i}+\mathbb{N} \tau_{i}$ meets both $\mathbb{Q}_{+} \tau$ and $\mathbb{Q}_{+} A \backslash \mathbb{Q}_{+} \tau$. Let $\ell \in \operatorname{Hom}_{\mathbb{Q}}\left(\mathbb{Q}^{d}, \mathbb{Q}\right)$ be a separating linear form, $\ell(\tau) \geq 0$ and $\ell\left(\tau_{i}\right) \leq 0$. If $\tau_{i} \subseteq \tau$ (and hence $\mathbf{u}_{i} \notin \mathbb{Q}_{+} \tau$ ), we chose $\mathbf{u}_{i}^{\prime} \in$ $\left(\mathbf{u}_{i}+\mathbb{N} \tau_{i}\right) \cap \mathbb{Q}_{+} \tau$. The submodule $S_{\tau_{i}}\left(-\mathbf{u}_{i}^{\prime}\right)$ of $S_{\tau_{i}}\left(-\mathbf{u}_{i}\right)$ has its $\mathbb{Z}^{d}$-degrees entirely in $\mathbb{Q}_{+} \tau$, and the cokernel of the inclusion has smaller dimension. If conversely $\tau_{i} \not \subset \tau$, choose $\ell \in \operatorname{Hom}_{\mathbb{Q}}\left(\mathbb{Q}^{d}, \mathbb{Q}\right)$ above such that $\ell\left(\tau_{i}\right) \neq 0$ and pick $\mathbf{u}_{i}^{\prime} \in \mathbf{u}_{i}+\mathbb{N} \tau_{i}$ with $\ell\left(\mathbf{u}_{i}^{\prime}\right)<0$. Then $S_{\tau_{i}}\left(-\mathbf{u}_{i}^{\prime}\right) \subseteq S_{\tau_{i}}\left(-\mathbf{u}_{i}\right)$ and has degrees completely outside of $\mathbb{Q}_{+} \tau$. 
Iterating this procedure we arrive at a composition chain as claimed. Then, however, the statement of the lemma is obvious: for each $i \in\{1, \ldots, l\}$ with $\operatorname{deg}\left(S_{\tau_{i}}\left(-\mathbf{u}_{i}\right)\right) \cap \mathbb{Q}_{+} \tau \neq \emptyset, \tau_{i} \subseteq \tau$, and $\left(S_{\tau_{i}}\left(-\mathbf{u}_{i}\right)\right)(\tau) \cong S_{\tau_{i}}$ is even module-finite over the subring $\mathbb{C}\left[\mathbb{N} \cdot k \cdot\left(\tau_{i} \cap \Phi_{A}^{L, 0}\right)\right]$ of $\mathbb{C}\left[\mathbb{N} \cdot k \cdot\left(\tau \cap \Phi_{A}^{L, 0}\right)\right]$.

Theorem 2.20. If $S_{A}^{L}(\tau)$ is Cohen-Macaulay for all $\tau \in \Phi_{A}^{L}$ not contained in the topological boundary of $\Gamma_{A}^{L}$ then $S_{A}^{L}$ is Cohen-Macaulay.

Proof. There is an integer $k>0$ such that $k \cdot \Gamma_{A}^{L}$ has vertex set $k \cdot \Gamma_{A}^{L, 0} \subseteq \mathbb{N} A$. Within the space of sequences $f_{1}, \ldots, f_{d}$ in $S_{A}^{L}$ for which each $f_{i}$ is a sum of terms whose $\mathbb{Z}^{d}$-degrees are in $k \cdot \Gamma_{A}^{L, 0}$, choose one sequence that is generic.

Each $f_{i}$ is homogeneous with respect to $N=N_{A}^{L}$ and can hence be identified with its $N$-symbol in $\operatorname{gr}^{N}\left(S_{A}^{L}\right)$. By Lemma 2.19, the vector space spanned by $\gamma_{A, \tau}\left(f_{1}\right), \ldots, \gamma_{A, \tau}\left(f_{d}\right) \in \mathbb{C}[\mathbb{N} \tau]$ contains a system of parameters on $S_{A}^{L}(\tau)$ for all $\tau \in \Phi_{A}^{L}$. This system of parameters is a regular sequence by the Cohen-Macaulay hypothesis. By the (spectral sequence) argument in Kou76, §2.12], the Koszul complex induced by $f_{1}, \ldots, f_{d}$ on the complex (2.8.1) with $V=S_{A}^{L}$ is a resolution. It follows that the Koszul complex induced by $f_{1}, \ldots, f_{d}$ on $\operatorname{gr}^{N}\left(S_{A}^{L}\right)$, and hence on $S_{A}^{L}$, is a resolution as well. Therefore, $f_{1}, \ldots, f_{d}$ is a regular sequence on $S_{A}^{L}$ and the claim follows.

\section{Characteristic VARIETy OF the hypergeometriC System}

3.1. Characteristic varieties and slopes. The Weyl algebra $D=\mathbb{C}[x]\langle\partial\rangle$ in $n$ variables $x=x_{1}, \ldots, x_{n}$ is the ring of $\mathbb{C}$-linear differential operators on $X=\mathbb{C}^{n}$ and contains $R$ as a commutative subring. The $\mathbb{Z}^{d}$-grading on $R$ extends to $D$ by setting $-\operatorname{deg}\left(x_{i}\right)=\mathbf{a}_{i}=\operatorname{deg}\left(\partial_{i}\right)$.

With $L_{x}=\left(L_{x_{1}}, \ldots, L_{x_{n}}\right)$ and $L_{\partial}=\left(L_{\partial_{1}}, \ldots, L_{\partial_{n}}\right), L=\left(L_{x}, L_{\partial}\right) \in \mathbb{Q}^{2 d}$ is a weight vector on $D$ if $L_{x}+L_{\partial} \geq 0$. Fix any such weight vector $L$; it defines an increasing filtration $L$ on $D$ by $\left[x^{\mathbf{u}} \partial^{\mathbf{v}} \in L_{k} D\right] \Leftrightarrow[L \cdot(\mathbf{u}, \mathbf{v}) \leq k]$. Since the Gröbner fan of any $D$-ideal is defined over $\mathbb{Q}$ (see ACJG00), the study of real weights can be reduced to the present rational case. If $P \in L_{k} D \backslash L_{<k} D$ then $k=: \operatorname{deg}^{L}(P)$ is the $L$-degree of $P$. The multiplicative, but not additive, $L$-symbol map

$$
\sigma^{L}: D \longrightarrow \operatorname{gr}^{L}(D)=: W
$$

is defined by $\sigma^{L}(P)=P \bmod L_{<k} R$ if $\operatorname{deg}^{L}(P)=k$. An element of the form $P=\sum_{L \cdot(\mathbf{u}, \mathbf{v})=k} P_{\mathbf{u}, \mathbf{v}} x^{\mathbf{u}} \partial^{\mathbf{v}} \in D$ with $P_{\mathbf{u}, \mathbf{v}} \in \mathbb{C}$ is $L$-homogeneous. By abuse of notation, we identify $L$-homogeneous elements in $D$ with their image under $\sigma^{L}$ in $W$. We restrict ourselves to the case $L_{x}+L_{\partial}>0$ in which case $W \cong \mathbb{C}[x, \partial]$ can be considered as the ring of polynomial functions on the cotangent space $T^{*} X$

The definition of the characteristic variety of a $D$-module is based on the concept of good filtrations discussed in more detail in Section 4 and Sch85, Ch. II, §§1.11.3]. Let $F$ be a filtration on a ring $T$. Then $G$ is called a good $F$-filtration on a $T$-module $N$ (see Sch85, Ch. II, Def. 1.1.1]), if there are generators $n_{1}, \ldots, n_{m}$ of $N$ and $\mathbf{u} \in \mathbb{Z}^{m}$ such that for all $k$ one has

$$
G_{k} N=\sum_{i=1}^{m} F_{k+u_{i}} T \cdot n_{i} .
$$


Note that good $F$-filtrations on $N$ exist if and only if $N$ is $T$-finite. From the definition follows the fact that all good $F$-filtrations on $N$ are equivalent in the sense that for all $k, l \in \mathbb{N}$ there are $k_{l}, l_{k} \in \mathbb{N}$ with $G_{k} N \subseteq G_{l_{k}}^{\prime} N$ and $G_{l}^{\prime} \subseteq G_{k_{l}}$.

Definition 3.1. The L-characteristic variety $\mathrm{Ch}^{L}(M)$ of a finite $D$-module $M$ on $X$ is the support of $\operatorname{gr}^{L}(M)$ in $T^{*} X$ for some good $L$-filtration on $M$. A finite $D$-module $M$ is L-holonomic if $\operatorname{dim} \mathrm{Ch}^{L}(M)=n$.

The independence of $\mathrm{Ch}^{L}(M)$ of the choice of the good $L$-filtration on $M$ follows from [Sch85, Ch. II, Prop. 1.3.1.a]. The following algebraic statement is a special case of a result by G.G. Smith Smi01, Thm. 1.1].

Theorem 3.2. The dimension of any component of $\mathrm{Ch}^{L}(M)$ is at least $n$.

Important special cases of filtrations on $D$ are the the order filtration $F=$ $\left(F_{x}, F_{\partial}\right)=(\mathbf{0}, \mathbf{1})$ and the $V$-filtration $V=\left(V_{x}, V_{\partial}\right)$ along the coordinate variety

$$
Y:=\operatorname{Var}\left(x_{\mathfrak{V}}\right) \subseteq X, \quad \mathfrak{V} \subseteq\{1, \ldots, n\},
$$

defined by $-V_{x_{i}}=1=V_{\partial_{i}}$ for $i \in \mathfrak{V}$ and $V_{x_{i}}=0=V_{\partial_{i}}$ for $i \notin \mathfrak{V}$. The notion of slopes along $Y$ (see Definition 3.4) involves the family of intermediate filtrations $L$ between $F$ and $V$ defined by the linear combination of weight vectors

$$
L=p F+q V, \quad p / q \in \mathbb{Q}_{>0} \cup\{\infty\} .
$$

Note that $L_{x}+L_{\partial}>0$ since $p>0$. If $p^{\prime} / q^{\prime}=p / q$ then the filtrations $L$ and $L^{\prime}$ are identical, up to a dilation in the index: $L_{p^{\prime} k}=L_{p k}^{\prime}$. By abuse of notation, we shall frequently identify the filtration $L$ with the number $p / q$. For $Y \subseteq X$ closed and reduced, let $T_{Y}^{*} X$ be (the closure of) the conormal bundle of (the smooth points of) $Y$ in $X$. With notation as in (3.1.1) and (3.1.2), the ring $W=\operatorname{gr}^{L}(D)$ can be considered as the ring of polynomial functions on the cotangent space $T^{*} T_{Y}^{*} X$ of $T_{Y}^{*} X$. For $i \in \mathfrak{V},-x_{i}$ can be interpreted as the partial derivative with respect to the variable $\partial_{i}$. This sets up an explicit isomorphism between $T^{*} T_{Y}^{*} X$ and $T^{*} X$.

Remark 3.3. For $L=p F+q V$ as in (3.1.2), $\mathrm{Ch}^{L}(M)$ is the global algebraic version of Y. Laurent's microcharacteristic variety $\Sigma_{\Lambda}^{r}(\mathcal{M})$ of type $r=p / q+1$ in $T^{*} \Lambda$ with $\Lambda=T_{Y}^{*} X$ (see [Lau87, §3.2]). Our algebraic $L$-filtration corresponds to the filtration on the sheaf of analytic differential operators on $X$ along $Y$ induced by $Y$. Laurent's microlocal filtration $F_{\Lambda, r}$ along $\Lambda$ (see Lau87, Def. 3.2.1]). By a flatness argument, A. Assi et al. ACJG96, Lem. 1.1.2] show that the analytification functor commutes with the grading by these two corresponding filtrations. Thus, the components of our $\mathrm{Ch}^{L}(M)$ which meet the preimage of $Y$ in $T^{*} X$ correspond to the components of $\Sigma_{\Lambda}^{r}(\mathcal{M})$.

The preceding remark motivated the following algebraic version of Y. Laurent's critical indices (see [Lau87, §3.4]) which are also called slopes.

Definition 3.4. For $L=p F+q V$ as in (3.1.2), we mean by $f\left(L^{\prime}\right)$ jumps at $L^{\prime}=L$ that the set-valued function

$$
\mathbb{Q} \ni p^{\prime} / q^{\prime} \longmapsto L^{\prime}=p^{\prime} F+q^{\prime} V \mapsto f\left(L^{\prime}\right)
$$

is not locally constant at $p / q$. A slope of a finite $D$-module $M$ at $y \in Y$ along $Y$ is a value $L=p / q$ such that the set of components of $\mathrm{Ch}^{L^{\prime}}(M)$ which meet $T_{y}^{*} X$ jumps at $L^{\prime}=L$. 
It follows from the existence of the Gröbner fan (see ACJG00) that a fixed $D$-module has only finitely many and only rational slopes along all coordinate varieties. Y. Laurent proved this finiteness and rationality along general varieties in the microlocal setting in [Lau87, Thm. 3.4.1]. He also showed that $\Sigma_{\Lambda}^{r}(\mathcal{M})$ is involutive (see [Lau87, Prop. 3.5.2]) and Lagrangian for holonomic $\mathcal{M}$ (see [Lau87, Cor. 4.1.2.(ii)]). In view of Remark 3.3, this implies for holonomic $M$ that $\mathrm{Ch}^{L}(M)$ is purely $n$-dimensional for $L=p F+q V$ as in (3.1.2).

3.2. Hypergeometric system and candidate components. We now define our main object of interest, the hypergeometric $D$-module $M_{A}(\beta)$, introduced in GGZ87, GZK89.

Definition 3.5. The Euler vector fields $E=\left(E_{1}, \ldots, E_{d}\right)$ of $A$ are defined by $E_{i}:=\sum_{j} a_{i, j} x_{j} \partial_{j}$ for $i=1, \ldots, d$. The A-hypergeometric (or GKZ) system defined by $A$ and a complex parameter vector $\beta \in \mathbb{C}^{d}$ is the $D$-module

$$
M_{A}(\beta):=D / D\left\langle I_{A}, E-\beta\right\rangle
$$

on $X$ defined by the toric ideal $I_{A}$ and the Euler operators $E-\beta$.

The $A$-hypergeometric system is always holonomic (see Ado94). Our goal is to understand its $L$-characteristic varieties and slopes under the assumption that

$$
L_{x}+L_{\partial}=(c, \ldots, c)=: \mathbf{c} \text { for some rational } c>0 .
$$

This guarantees that $W$ is a polynomial ring and $E$ is $L$-homogeneous of positive $L$-degree. Note that $M_{A}(\beta)$ is $L$-homogeneous if $A$ is $L$-homogeneous as defined in Definition 2.7. The following statement is a consequence of Buchberger's algorithm.

Lemma 3.6. One has the identity $\operatorname{gr}^{L}\left(D I_{A}\right)=W I_{A}^{L}$.

The vector fields $t_{1} \partial_{t_{1}}, \ldots, t_{d} \partial_{t_{d}}$ span the tangent space at any point of the torus $\mathbb{T}=\left(\mathbb{C}^{*}\right)^{d}$. Hence, for any $\xi \in T_{0}^{*} X$, the tangent space of the orbit $\operatorname{Orb}(\xi)$ is spanned by the pushforwards $E_{i}^{T}:=\sum_{j} a_{i, j} \partial_{j}\left(-x_{j}\right)$ of $t_{i} \partial_{t_{i}}$ under the map $\mathbb{T} \rightarrow T_{0}^{*} X, t \mapsto t \cdot \xi$ from (2.1.1). Since $E_{i}$ is $L$-homogeneous of positive degree with $\sigma^{L}\left(E_{i}\right)=-\sigma^{L}\left(E_{i}^{T}\right)$, the equations $\sigma^{L}(E-\beta)=0$ impose the conormal condition to the orbit $\operatorname{Orb}(\xi) \subseteq T_{0}^{*} X$ in $T^{*} T_{0}^{*} X=T^{*} X$. In what follows we abuse notation by writing $\sigma^{L}(E)=E$.

Definition 3.7. For a subset $\tau$ of $\{1, \ldots, n\}$, we denote by $C_{A}^{\tau} \subseteq T^{*} X$ the conormal space to the orbit $O_{A}^{\tau} \subseteq T_{0}^{*} X$ from Definition 2.2. We denote by $P_{C} \subseteq W$ the defining ideal of any irreducible variety $C \subseteq T^{*} X$ and abbreviate $P_{C_{A}^{\tau}}$ by $P_{\tau}$.

Proposition 3.8. The L-characteristic variety of $M_{A}(\beta)$ is

$$
\mathrm{Ch}^{L}\left(M_{A}(\beta)\right)=\bigcup_{\tau \in \phi_{A}^{L}(\beta)} \bar{C}_{A}^{\tau}=\bigsqcup_{\tau \in \phi_{A}^{L}(\beta)} C_{A}^{\tau}
$$

for some subset $\phi_{A}^{L}(\beta) \subseteq \Phi_{A}^{L}$. In particular, the hypergeometric system $M_{A}(\beta)$ is $L$-holonomic for any $L$.

Proof. By definition, $\mathrm{Ch}^{L}\left(M_{A}(\beta)\right) \subseteq \operatorname{Var}\left(W\left\langle I_{A}^{L}, E\right\rangle\right)$. By Theorem 2.14 and the preceding arguments,

$$
\operatorname{Var}\left(W\left\langle I_{A}^{L}, E\right\rangle\right)=\bigsqcup_{\tau \in \Phi_{A}^{L}} C_{A}^{\tau}=\bigcup_{\tau \in \Phi_{A}^{L}} \bar{C}_{A}^{\tau}
$$


Then Theorem 3.2 assures that $\mathrm{Ch}^{L}\left(M_{A}(\beta)\right)$ is purely $n$-dimensional and hence a union of closures of conormals $C_{A}^{\tau}$ for certain $\tau \in \Phi_{A}^{L}$.

3.3. Existence of facet components. In this subsection, we use an elementary localization argument to give an index formula for the multiplicity of components in the characteristic cycle $\mathrm{CC}^{L}\left(M_{A}(\beta)\right)$ (see Definition 4.4) corresponding to facets in the $(A, L)$-umbrella. It shows in particular that these multiplicities are independent of $\beta$ and positive, and that all facet components occur in $\mathrm{Ch}^{L}\left(M_{A}(\beta)\right)$. We deduce from this a converse to R. Hotta's Theorem: regular $A$-hypergeometric systems are homogeneous in the usual sense.

Definition 3.9. We denote by $\mu_{A, 0}^{L, C}(\beta)$ the multiplicity of $\operatorname{gr}^{L}\left(M_{A}(\beta)\right)$ along $C$. (The reason for the appearance of the subscript " 0 " will become apparent in Section 4.) This is the length of the $W_{P_{C}}$-module $\operatorname{gr}^{L}\left(M_{A}(\beta)\right) \otimes_{W} W_{P_{C}}$. We write $\mu_{A, 0}^{L, \tau}(\beta)$ if $C=\bar{C}_{A}^{\tau}$.

Theorem 3.10. For all $\beta \in \mathbb{C}^{d}$ and all $\tau \in \Phi_{A}^{L, d-1}$,

$$
\mu_{A, 0}^{L, \tau}(\beta)=\nu_{A}^{L, \tau}=\left[\mathbb{Z}^{d}: \mathbb{Z} \tau\right]
$$

In particular, $\Phi_{A}^{L, d-1} \subseteq \phi_{A}^{L}(\beta)$ for all $\beta \in \mathbb{C}^{d}$.

Proof. Let $\tau \in \Phi^{L, d-1}$ and relabel columns such that $\mathbf{a}_{1}^{L}, \ldots, \mathbf{a}_{d}^{L} \in \tau$ and such that $\mathbf{a}_{1}, \ldots, \mathbf{a}_{d}$ are linearly independent. We have to show that $C_{A}^{\tau} \subseteq \mathrm{Ch}^{L}\left(M_{A}(\beta)\right)$. First, we verify that $E$ is a regular sequence on $W\left[\partial_{\tau}^{-1}\right] / W\left[\partial_{\tau}^{-1}\right] I_{A}^{L}$. After Gauss reduction on $E$ and multiplying $E_{i}$ by $\partial_{i}^{-1}, E_{i} \equiv x_{i}$ modulo terms independent of $x_{j}$ for all $j \leq d$. After a change of the coordinates $x_{1}, \ldots, x_{d}$ in $W\left[\partial_{\tau}^{-1}\right]$, leaving $I_{A}^{L}$ invariant, $E_{i}=x_{i}$. As $I_{A}^{L}$ does not involve the variables $x_{1}, \ldots, x_{d}, E$ is a regular sequence modulo $W\left[\partial_{\tau}^{-1}\right] I_{A}^{L}$ as claimed.

Since $W\left[\partial_{\tau}^{-1}\right] I_{A}^{L}=\operatorname{gr}^{L}\left(D\left[\partial_{\tau}^{-1}\right] I_{A}\right)$ by Lemma 3.11 below, $\operatorname{gr}^{L}\left(D\left[\partial_{\tau}^{-1}\right]\left\langle I_{A}, E-\beta\right\rangle\right)=$ $\operatorname{gr}^{L}\left(D\left[\partial_{\tau}^{-1}\right] I_{A}\right)+\operatorname{gr}^{L}\left(D\left[\partial_{\tau}^{-1}\right]\langle E\rangle\right)$ by the argument in [SST00, Thm. 4.3.5]. Again by Lemma 3.11 .

$$
\operatorname{gr}^{L}\left(M_{A}(\beta)\right)\left[\partial_{\tau}^{-1}\right]=W\left[\partial_{\tau}^{-1}\right] / W\left[\partial_{\tau}^{-1}\right]\left\langle I_{A}^{L}, E\right\rangle .
$$

Since $\partial_{j} \notin P_{\tau}$ for all $j \in \tau$, this yields

$$
\operatorname{gr}^{L}\left(M_{A}(\beta)\right)_{P_{\tau}}=W_{P_{\tau}} / W_{P_{\tau}}\left\langle I_{A}^{L}, E\right\rangle \cong\left(R_{I_{A}^{\tau}} / I_{A}^{L}\right)\left(x_{d+1}, \ldots, x_{n}\right)
$$

with the ring isomorphism defined by the above coordinate change, and hence

$$
\mu_{A, 0}^{L, \tau}(\beta)=\ell\left(\operatorname{gr}^{L}\left(M_{A}(\beta)\right)_{P_{\tau}}\right)=\ell\left(R_{I_{A}^{\tau}} / I_{A}^{L}\right)=\nu_{A}^{L, \tau} .
$$

Then Proposition 2.17 finishes the proof.

Lemma 3.11. For $I \subseteq D$ and $\tau \subseteq\{1, \ldots, n\}, \operatorname{gr}^{L}\left(D\left[\partial_{\tau}^{-1}\right] I\right)=\operatorname{gr}^{L}\left(D\left[\partial_{\tau}^{-1}\right]\right) \operatorname{gr}^{L}(I)$. In particular, $\operatorname{gr}^{L}\left((D / I)\left[\partial_{\tau}^{-1}\right]\right)=\left(\operatorname{gr}^{L}(D / I)\right)\left[\partial_{\tau}^{-1}\right]$.

Proof. The inclusion $\operatorname{gr}^{L}\left(D\left[\partial_{\tau}^{-1}\right] I\right) \supseteq \operatorname{gr}^{L}\left(D\left[\partial_{\tau}^{-1}\right]\right) \operatorname{gr}^{L}(I)$ holds trivially. For $Q \in$ $D\left[\partial_{\tau}^{-1}\right], \partial_{\tau}^{\mathbf{u}} Q \in D$ for some $\mathbf{u} \in \mathbb{N}^{|\tau|}$. If $\left\{P_{i}\right\}$ is a finite set of generators for $I$ then the opposite inclusion follows from

$$
\sigma^{L}\left(\sum_{i} Q_{i} P_{i}\right)=\partial_{\tau}^{-\mathbf{u}} \sigma^{L}\left(\sum_{i} \partial_{\tau}^{\mathbf{u}} Q_{i} P_{i}\right) \in \operatorname{gr}^{L}\left(D\left[\partial_{\tau}^{-1}\right]\right) \operatorname{gr}^{L}(I)
$$


with a common $\mathbf{u}$ for the finitely many $Q_{i} \in D\left[\partial_{\tau}^{-1}\right]$. In particular,

$$
\begin{gathered}
\operatorname{gr}^{L}\left((D / I)\left[\partial_{\tau}^{-1}\right]\right)=\operatorname{gr}^{L}\left(D\left[\partial_{\tau}^{-1}\right] / D\left[\partial_{\tau}^{-1}\right] I\right)=\operatorname{gr}^{L}\left(D\left[\partial_{\tau}^{-1}\right]\right) / \operatorname{gr}^{L}\left(D\left[\partial_{\tau}^{-1}\right]\right) \operatorname{gr}^{L}(I) \\
=W\left[\partial_{\tau}^{-1}\right] / W\left[\partial_{\tau}^{-1}\right] \operatorname{gr}^{L}(I)=\left(W / \operatorname{gr}^{L}(I)\right)\left[\partial_{\tau}^{-1}\right]=\left(\operatorname{gr}^{L}(D / I)\right)\left[\partial_{\tau}^{-1}\right]
\end{gathered}
$$

Example 3.12. Let $A$ be as in Figure 1; we consider $L=F+t V$ where $V$ is the $V$-filtration along $\operatorname{Var}\left(x_{4}\right)$, induced by the weight vector $(0,0,0,-1,0,0,0,1)$. We consider specifically $t \in\{0,1,4\}$. As these weights are generic, for all $\tau \subseteq\{1, \ldots, 4\}$ the conormal closures $\bar{C}_{A}^{\tau}$ are coordinate subspaces $\operatorname{Var}\left(x_{\tau}, \partial_{\bar{\tau}}\right)$. The facets in $\Phi_{A}^{L}$ for these values of $t$ are shown in Figure 1

For $t=0$ one finds $\mu_{A, 0}^{L,\{1,4\}}(\beta)=12$ and $\mu_{A, 0}^{L,\{2,4\}}(\beta)=1$ with sum $13=$ $\operatorname{vol}_{\mathbb{Z}^{d}}\left(\Delta_{A}^{F}\right.$ ) (for relevant notation and more information, see Definition 4.19 and the continuation in Example 4.24). For $t=1$ one finds three facets, with $\mu_{A, 0}^{L,\{1,3\}}(\beta)=$ $3, \mu_{A, 0}^{L,\{3,4\}}(\beta)=7$ and $\mu_{A, 0}^{L,\{2,4\}}(\beta)=1$. On the other hand, $t=4$ yields two facets only, with $\mu_{A, 0}^{L,\{1,3\}}(\beta)=3$ and $\mu_{A, 0}^{L,\{2,3\}}(\beta)=2$. The respective sums, $3+7+1$ and $3+2$, are the degrees of the $L$-graded toric ideals (see Proposition 2.17).

Lemma 3.13. The map $L^{\prime} \mapsto \Phi_{A}^{L^{\prime}, d-1}$ jumps at $L^{\prime}=L$ if and only if the map $L^{\prime} \mapsto \Phi_{A}^{L^{\prime}}$ jumps at $L^{\prime}=L$.

Proof. The "only if" part in the statement follows trivially from $\Phi_{A}^{L^{\prime}, d-1} \subseteq \Phi_{A}^{L^{\prime}}$. Assume that $\Phi_{A}^{L^{\prime}, d-1}$ is locally constant at $L$ and let $\tau \in \Phi_{A}^{L}$. Then there is a $\tau^{\prime} \in \Phi_{A}^{L, d-1}$ such that $\tau$ is a face of $\tau^{\prime}$. By assumption, $\tau^{\prime} \in \Phi_{A}^{L^{\prime}, d-1}$ for $L^{\prime}$ close to $L$. Since $\tau^{\prime}$ is not contained in a hyperplane through origin and since the $\mathbf{a}_{j}^{L^{\prime}}$ depend on $L^{\prime}$ only by scaling, $\tau$ remains a face of $\tau^{\prime}$ for $L^{\prime}$ close to $L$. In particular, $\tau \in \Phi_{A}^{L^{\prime}}$ for $L^{\prime}$ close to $L$ and hence $\Phi_{A}^{L^{\prime}}$ is locally constant at $L$.

Theorem 3.10 and Lemma 3.13 yield the existence of slopes of $M_{A}(\beta)$ corresponding to jumps of the $(A, L)$-umbrella with $L$ as in (3.1.2). The following result on the candidate components $\bar{C}_{A}^{\tau}$ of $\mathrm{Ch}^{L}\left(M_{A}(\beta)\right)$ allows us to show that all these slopes occur at the origin.

Lemma 3.14. For all $\tau \in \Phi_{A}^{L}$, the candidate component $\bar{C}_{A}^{\tau}$ meets $T_{0}^{*} X$.

Proof. Since the ideal $W\left\langle I_{A}^{L}, E\right\rangle$ is homogeneous in the $x$-variables, this holds also for its associated prime ideals $P_{\tau}$ where $\tau \in \Phi_{A}^{L}$. Thus $\bar{C}_{A}^{\tau}=\operatorname{Var}\left(P_{\tau}\right)$ meets $T_{0}^{*} X$ as claimed.

Corollary 3.15. For $L=p F+q V$ as in (3.1.2), if $\Phi_{A}^{L}$ jumps at $L=L^{\prime}$ then $L^{\prime}=p^{\prime} / q^{\prime}$ is a slope of $M_{A}(\beta)$ along $Y$ at $0 \in Y$.

In particular, we obtain the following converse to a Theorem by R. Hotta Hot98, Ch. II, §6.2, Thm.].

Corollary 3.16. Regular A-hypergeometric systems are homogeneous with respect to the order filtration $F$.

Proof. The polytope $\operatorname{conv}(A)$ is an intersection of closed half-spaces and $0 \notin \operatorname{conv}(A)$ by positivity of $\mathbb{N} A$. Thus there is a facet $\tau$ of $\operatorname{conv}(A)$ and a linear form $\ell \in$ $\operatorname{Hom}_{\mathbb{Q}}\left(\mathbb{Q}^{d}, \mathbb{Q}\right)$ such that $\ell\left(\mathbf{a}_{i}\right) \geq 1$ with equality equivalent to $i \in \tau$. If $\tau \in \Phi_{A}^{F, d-1}$ then $\tau$ is a facet of $\Delta_{A}^{F}=\operatorname{conv}\left(\left\{0, \mathbf{a}_{1}, \ldots, \mathbf{a}_{n}\right\}\right)$. But then $0 \in \Delta_{A}^{F}$ and $\ell(0)=0<1$ 
implies that $\ell\left(\mathbf{a}_{i}\right) \leq 1$ and hence $\ell\left(\mathbf{a}_{i}\right)=1$ for $i=1, \ldots, n$. So in this case $A$ is $F$-homogeneous. Assume now that $\tau \notin \Phi_{A}^{F, d-1}$ and let $L=F+\frac{q}{p} V$ where $\mathfrak{V}=\bar{\tau}$ in (3.1.1). For $i \in \tau, \mathbf{a}_{i}^{L}=\mathbf{a}_{i}$ and hence $\ell\left(\mathbf{a}_{i}^{L}\right)=1$. But for $i \notin \tau$ and $p / q \rightarrow 0$, $\mathbf{a}_{i}^{L} \rightarrow 0$ and hence eventually $\ell\left(\mathbf{a}_{i}^{L}\right)<1$. Thus, $\tau \in \Phi_{A}^{L, d-1}$ for $p / q \rightarrow 0$ while $\tau \notin \Phi_{A}^{F, d-1}$ and hence $L \mapsto \Phi_{A}^{L}$ can not be constant. Then, by Corollary 3.15, $M_{A}(\beta)$ has slopes. By [LM99, Thm. 2.4.2], $M_{A}(\beta)$ is hence irregular.

We will see later in Corollary 4.18 that Corollary 3.15 actually gives a complete list of all slopes along coordinate varieties at the origin.

Remark 3.17. For generic $\beta$, the equivalence of the regularity of $M_{A}(\beta)$ with the homogeneity of $A$ was already obtained in [SST00, Thm. 2.4.11].

\section{Characteristic cycle of the Euler-Koszul complex}

In this section we assume that $L_{x}+L_{\partial}=\mathbf{c}$ is a constant positive vector as in (3.2.1). By way of a dilation we further may, and do, assume that the index set of the $L$-filtration is $\mathbb{Z}$.

By Proposition 3.8 all components of the $L$-characteristic variety of $M_{A}(\beta)$ are of the form $\bar{C}_{A}^{\tau}$ where $\tau \in \Phi_{A}^{L}$. In this section we prepare the way for Corollary 4.17 which ascertains the presence of every such candidate component. The approach is to consider $M_{A}(\beta)$ as the 0 -th homology of a Koszul type complex by operators $E-\beta$ on the $D$-module $D \otimes_{R} N$ for the $\mathbb{Z}^{d}$-graded $R$-module $N=S_{A}$. For modules $N$ having a composition series with quotients of type $S_{\theta}, \theta \in \Phi_{A}^{0}$, we can apply results in Sections 2 and 3 combined with homological algebra. For basic results on filtered rings and modules we refer to [Sch85, Ch. II, §§1.1-1.3].

4.1. Good filtrations and toric modules. In order to combine homological methods with good $L$-filtrations on $D$-modules, we need the $L$-filtration on $D$ to be Noetherian, which means the following.

The Rees ring of a ring $T$ with a $\mathbb{Z}$-indexed filtration $F$ is the graded ring

$$
\operatorname{Rees}^{F}(T)=\bigoplus_{i \in \mathbb{Z}} F_{i}(T) t^{i} \subseteq T\left[t, t^{-1}\right]
$$

and $F$ is called Noetherian if $\operatorname{Rees}^{F}(T)$ is Noetherian.

Lemma 4.1. The filtrations $L$ on $D$ and $L_{\partial}$ on $R$ are Noetherian.

Proof. Let us write $(\mathbf{u}, \mathbf{v}):=\left(L_{x}, L_{\partial}\right)$, then $\mathbf{u}+\mathbf{v}=\mathbf{c}>0$ by hypothesis. The elements of Rees ${ }^{L}(D)$ are of the form $\sum_{k \in \mathbb{Z}} \sum_{\mathbf{u} \cdot \mathbf{u}^{\prime}+\mathbf{v} \cdot \mathbf{v}^{\prime} \leq k} c_{\mathbf{u}^{\prime}, \mathbf{v}^{\prime}, k} x^{\mathbf{u}^{\prime}} \partial^{\mathbf{v}^{\prime}} t^{k}$. Apply the change of variables $x_{i} \mapsto x_{i} t^{-u_{i}}, \partial_{i} \mapsto \partial_{i} t^{u_{i}}$ and set $m=k+\mathbf{u} \cdot\left(\mathbf{v}^{\prime}-\mathbf{u}^{\prime}\right)$. Then the elements are transformed to $\sum_{m \in \mathbb{Z}} \sum_{(\mathbf{v}+\mathbf{u}) \cdot \mathbf{v}^{\prime} \leq m} x^{\mathbf{u}^{\prime}} \partial^{\mathbf{v}^{\prime}} t^{m}$. Thus, $\operatorname{Rees}^{L}(D) \cong$ $\operatorname{Rees}^{F^{\prime}}(D)$ where $F^{\prime}:=(0, \mathbf{v}+\mathbf{u})=(0, \mathbf{c})$, which is Noetherian by Sch85. Ch. II, Prop. 1.1.8] since $F_{0}^{\prime} D=\mathbb{C}[x]$ is Noetherian. Similarly, a change of variables $\partial_{i} \mapsto \partial_{i} t^{1-v_{i}}$ shows that $\operatorname{Rees}^{L_{\partial}}(R) \cong \operatorname{Rees}^{F_{\partial}}(R)$ is Noetherian.

The Rees module of a $T$-module $N$ with an $F$-filtration $G$ is the graded $\operatorname{Rees}^{F}(T)$ module

$$
\operatorname{Rees}^{G}(N)=\bigoplus_{i \in \mathbb{Z}} G_{i}(N) t^{i} \subseteq N\left[t, t^{-1}\right]
$$


For Noetherian $F, G$ is a good $F$-filtration on $N$ in the sense of Subsection 3.1 if and only if $\operatorname{Rees}^{G}(N)$ is Noetherian over $\operatorname{Rees}^{F}(T)$, (see [Sch85, Ch. II, Prop. 1.1.7]). The advantage of this new definition is the following. Consider a short exact sequence of $T$-modules

$$
0 \longrightarrow N^{\prime} \longrightarrow N \longrightarrow N^{\prime \prime} \longrightarrow 0
$$

a good $F$-filtration $G$ on $N$, and the induced $F$-filtrations $G^{\prime}$ on $N^{\prime}$ and $G^{\prime \prime}$ on $N^{\prime \prime}$. Then the associated Rees sequence

$$
0 \longrightarrow \operatorname{Rees}^{G^{\prime}}\left(N^{\prime}\right) \longrightarrow \operatorname{Rees}^{G}(N) \longrightarrow \operatorname{Rees}^{G^{\prime \prime}}\left(N^{\prime \prime}\right) \longrightarrow 0
$$

is exact and hence $G^{\prime}$ and $G^{\prime \prime}$ are good according to the new definition. By definition of $G^{\prime}$ and $G^{\prime \prime}$, the maps in the sequence (4.1.1) are strict and hence

$$
0 \longrightarrow \operatorname{gr}^{G^{\prime}}\left(N^{\prime}\right) \longrightarrow \operatorname{gr}^{G}(N) \longrightarrow \operatorname{gr}^{G^{\prime \prime}}\left(N^{\prime \prime}\right) \longrightarrow 0
$$

is exact.

For $D$-modules of the type $M=D \otimes_{R} N$ where $N$ is an $R$-module, a good $L_{\partial}$-filtration on $N$ induces a good $L$-filtration on $M$ by

$$
L_{k}\left(D \otimes_{R} N\right)=\sum_{i+j=k}\left(L_{x}\right)_{i} \mathbb{C}[x] \otimes_{\mathbb{C}}\left(L_{\partial}\right)_{j} N .
$$

Here, $\left(L_{x}\right)_{i} \mathbb{C}[x]$ denotes the level- $i$ piece of the filtration $L_{x}$ on $\mathbb{C}[x]$. For many purposes, we can replace a given good $L$-filtration on $M$ by that in (4.1.2) and then Lemma 3.6 generalizes as follows.

Lemma 4.2. For any $R$-module $N$, $\operatorname{gr}^{L}\left(D \otimes_{R} N\right)=W \otimes_{R} \operatorname{gr}^{L_{\partial}}(N)$ as $W$-modules.

The following definition (see MMW05, Def. 4.5]) describes a class of $R$-modules that arise naturally in the study of $A$-hypergeometric systems. Recall that, by Theorem 2.14, the $\mathbb{Z}^{d}$-graded prime ideals containing $I_{A}$ are of the form $I_{A}^{\theta}$ for $\theta \in \Phi_{A}^{0}$.

Definition 4.3. A $\mathbb{Z}^{d}$-graded $R$-module $N$ is toric if it has a toric filtration

$$
0=N_{0} \subsetneq N_{1} \subsetneq \cdots \subsetneq N_{l-1} \subsetneq N_{l}=N
$$

which means that for all $i, N_{i} / N_{i-1}=S_{\theta_{i}}\left(\mathbf{u}_{i}\right)$ for some $\theta_{i} \in \Phi_{A}^{0}$ and some $\mathbf{u}_{i} \in \mathbb{Z}^{d}$. The minimal such $l$ is called the toric length of $N$. A toric morphism is a $\mathbb{Z}^{d}$-graded $R$-linear map between toric modules.

4.2. Characteristic cycle and Euler-Koszul homology. Recall that by Theorem 3.2 any component of the $L$-characteristic variety $\mathrm{Ch}^{L}(M)$ of a finite $D$-module $M$ is at least $n$-dimensional.

Definition 4.4. The $L$-characteristic cycle of an $L$-holonomic $D$-module $M$ is the formal sum of $n$-dimensional irreducible varieties in $T^{*} X$

$$
\mathrm{CC}^{L}(M):=\sum_{C} \mu^{L, C}(M) \cdot C, \quad \mu^{L, C}(M):=\ell\left(\operatorname{gr}^{L}(M)_{P_{C}}\right),
$$

for some good $L$-filtration on $M$. 
A bounded complex of $D$-modules $K_{\bullet}$ is called homologically L-holonomic if all its homology modules $H_{i}\left(K_{\bullet}\right)$ are $L$-holonomic. The $L$-characteristic cycle of a homologically $L$-holonomic complex of finite $D$-modules $K_{\bullet}$ is

$$
\mathrm{CC}^{L}\left(K_{\bullet}\right):=\sum_{i \in \mathbb{Z}}(-1)^{i} \mathrm{CC}^{L}\left(H_{i}\left(K_{\bullet}\right)\right)=\sum_{C} \mu^{L, C}\left(K_{\bullet}\right) \cdot C,
$$

where

$$
\mu^{L, C}\left(K_{\bullet}\right):=\sum_{i \in \mathbb{Z}}(-1)^{i} \mu_{i}^{L, C}\left(K_{\bullet}\right), \quad \mu_{i}^{L, C}\left(K_{\bullet}\right):=\ell\left(\operatorname{gr}^{L}\left(H_{i}\left(K_{\bullet}\right)\right)_{P_{C}}\right) .
$$

For an $L$-holonomic $D$-module $M$ considered as a complex with trivial differential concentrated in homological degree zero, the two definitions of $\mathrm{CC}^{L}(M)$ coincide.

A $D$-module is holonomic in the usual sense if it is $L$-holonomic for $L=F$. The independence of $\mathrm{CC}^{L}(M)$ of the particular choice of the good $L$-filtration follows from [Sch85, Ch. II, Prop. 1.3.1.a]. Essentially by definition, the $L$-characteristic variety $\mathrm{Ch}^{L}(M)$ of an $L$-holonomic $M$ in Definition 3.1 is the union

$$
\mathrm{Ch}^{L}(M)=\bigcup_{\mu^{L, C}(M)>0} C .
$$

For $L=p F+q V$ as in (3.1.2), $\mathrm{CC}^{L}(M)$ is the global algebraic version of Y. Laurent's microcharacteristic cycle $\widetilde{\Sigma}_{\Lambda}^{r}(\mathcal{M})$ of type $r=p / q+1$ in $T^{*} \Lambda$ with $\Lambda=$ $T_{Y}^{*} X$ whose support is $\Sigma_{\Lambda}^{r}(\mathcal{M})$ (see Remark 3.3). Lemma 4.1] and Sch85, Ch. II, Prop. 1.3.1.b] yield the following statement.

Lemma 4.5. The L-characteristic cycle $\mathrm{CC}^{L}$ is additive.

Our main technical tool for the study of $\mathrm{CC}\left(M_{A}(\beta)\right)$ is the Euler-Koszul functor from MMW05. For a $\mathbb{Z}^{d}$-graded left $D$-module $M$ and a $\mathbb{Z}^{d}$-homogeneous $y \in M$,

$$
\left(E_{i}-\beta_{i}\right) \circ y:=\left(E_{i}-\beta_{i}+\operatorname{deg}_{i}(y)\right) y
$$

defines $d$ commuting $D$-linear endomorphisms $E-\beta$ of $M$.

Definition 4.6. Let $N$ be a $\mathbb{Z}^{d}$-graded $R$-module and $\beta \in \mathbb{C}^{d}$. The Euler-Koszul complex $K_{A, \bullet}(N ; \beta)$ is the Koszul complex of the endomorphisms $E-\beta$ on the left $D$-module $D \otimes_{R} N$. Its $i$-th homology $H_{A, i}(N ; \beta):=H_{i}\left(K_{A, \bullet}(N ; \beta)\right)$ is the $i$-th Euler-Koszul homology of $N$.

To a good $L_{\partial}$-filtration on a $\mathbb{Z}^{d}$-graded $R$-module $N$ we associate a good $L$ filtration on $K_{A, \bullet}(N ; \beta)$ by setting for $i_{1}<\cdots<i_{k}$ the $L$-degree of the $\left(i_{1}, \ldots, i_{k}\right)$ th unit vector in $K_{A, k}(R ; \beta)=D^{\left(\begin{array}{c}n \\ k\end{array}\right)}$ equal to $\sum_{t=1}^{k} \operatorname{deg}^{L}\left(E_{i_{t}}\right)$, and using equation (4.1.2). Then Lemma 4.2 implies that

$$
\operatorname{gr}^{L}\left(K_{A, \bullet}(N ; \beta)\right)=W \otimes_{R} K_{\bullet}\left(\operatorname{gr}^{L}(N) ; E\right),
$$

by which, abusing notation, we mean the usual Koszul complex induced by the $L$-symbols of $E$ on $W \otimes_{R} \operatorname{gr}^{L}(N)$.

By [MMW05, Thm. 5.1], $K_{A, \bullet}(N ; \beta)$ is homologically $F$-holonomic for all toric modules $N$. Using Proposition 3.8 and Lemma 4.5. one shows by induction on the toric length of $N$ combined with a spectral sequence argument as in the proof of Theorem 4.11 that $K_{A, \bullet}(N ; \beta)$ is homologically $L$-holonomic for all toric modules $N$ and for all $L$. 
Definition 4.7. For a toric module $N$, we call

$$
\mathrm{CC}^{L}(N):=\mathrm{CC}^{L}\left(K_{A, \bullet}(N ; \beta)\right)=\sum_{i=0}^{d}(-1)^{i} \mathrm{CC}^{L}\left(H_{A, i}(N ; \beta)\right)
$$

the $L$-characteristic Euler-Koszul cycle of $N$. Recall Definition 3.9. We denote the $i$-th Euler-Koszul L-multiplicity and the Euler-Koszul L-characteristic of $N$ along $C$ by

$$
\mu_{A, i}^{L, C}(N ; \beta):=\mu_{i}^{L, C}\left(K_{A, \bullet}(N ; \beta)\right), \quad \mu_{A}^{L, C}(N):=\mu^{L, C}\left(K_{A, \bullet}(N ; \beta)\right) .
$$

If $N=S_{A}$ we skip the $\operatorname{argument} N$, while if $C=\bar{C}_{A}^{\tau}$ we write $\tau$ rather than $\bar{C}_{A}^{\tau}$ as upper index.

The independence of $\beta$ suggested by the notation $\mu_{A}^{L, C}(N)$ will be established in Theorem 4.11 below. Lemma 4.5 and the long exact Euler-Koszul homology sequence imply the following statement.

Lemma 4.8. The Euler-Koszul L-characteristic $\mu_{A}^{L, C}$ along $C$ is additive.

4.3. Euler-Koszul characteristic and intersection multiplicity. We now interpret $\mu_{A}^{L, \tau}$ as an intersection multiplicity (see [Ser65, Ch. V, $\left.\S \mathrm{B}\right]$ ). That will lead to an explicit formula in Theorem 4.21.

Definition 4.9. For two $W$-modules $M$ and $M^{\prime}$ and a variety $C \subseteq \operatorname{Spec}(W)$ not strictly contained in an irreducible component of $\operatorname{supp}\left(M \otimes_{W} M^{\prime}\right)$, the intersection multiplicity of $M$ and $M^{\prime}$ along $C$ is the alternating sum

$$
\chi^{C}\left(M, M^{\prime}\right):=\sum_{i}(-1)^{i} \ell\left(\operatorname{Tor}_{i}^{W_{P_{C}}}\left(M_{P_{C}}, M_{P_{C}}^{\prime}\right)\right)
$$

of lengths of $W_{P_{C}}$-modules. If $C=\bar{C}_{A}^{\tau}$ we write $\tau$ rather than $\bar{C}_{A}^{\tau}$ as upper index.

Lemmas 4.1 and 4.2 and [Sch85, Ch. II, Prop. 1.3.1.b] yield the following statements.

Lemma 4.10. Fix a $W$-module $M$ and a variety $C \subseteq \operatorname{Spec}(W)$. Whenever defined, the quantity $\chi^{C}\left(M, W \otimes_{R} \operatorname{gr}^{L}(N)\right)$ is independent of the choice of a good L-filtration on the R-module $N$ and additive in $N$.

Theorem 4.11. For any toric module $N$ and all $\beta \in \mathbb{C}^{d}$,

$$
\mu_{A}^{L, C}(N)=\chi^{C}\left(W / W\langle E\rangle, W \otimes_{R} \operatorname{gr}^{L}(N)\right)
$$

and is hence independent of $\beta$. For any $i \in \mathbb{N}, \mu_{A, i}^{L, C}(N ; \beta)>0$ only if $C=\bar{C}_{A}^{\tau}$ for some $\tau \in \Phi_{A}^{L}$, while $\mu_{A}^{L, C}(N)>0$ if and only if $\operatorname{dim}(N)=d$ and $C=\bar{C}_{A}^{\tau}$ for some $\tau \in \Phi_{A}^{L}$.

Proof. We consider $K_{\bullet}=K_{A, \bullet}(N ; \beta)$ and fix a variety $C$ for which $\mu_{A}^{L, C}(N)$ is defined. By Corollary A.2 and equation (4.2.2), there is a sequence of homologically graded $W_{P_{C}}$-modules $K^{1}, \ldots, K^{r}$ with $W_{P_{C}}$-linear endomorphisms $d^{i}: K^{i} \rightarrow K^{i}$ such that

$$
K^{1}=W_{P_{C}} \otimes_{R} K_{\bullet}\left(\operatorname{gr}^{L}(N) ; E\right),
$$

$K^{i+1}=H\left(K^{i}, d^{i}\right)$ for $i=1, \ldots, r-1$, and $K^{r}=\operatorname{gr}^{L}\left(H_{A, \bullet}(N ; \beta)\right)_{P_{C}}$ as homologically graded modules. Thus for all $\beta \in \mathbb{C}^{d}, \mu_{A}^{L, C}(N)=\chi\left(K^{r}\right)=\cdots=\chi\left(K^{1}\right)$ is independent of $\beta$ by additivity of $\chi(K):=\sum_{i}(-1)^{i} \ell\left(H_{i}(K, d)\right)$. Since $E$ is a 
regular sequence in $W$, Koszul homology against $E$ computes Tor over both $W$ and $W_{P_{C}}$ and hence

$$
\begin{aligned}
\chi\left(K^{1}\right) & =\sum_{i=1}^{d}(-1)^{i} \ell\left(\operatorname{Tor}_{i}^{W_{P_{C}}}\left(W_{P_{C}} / W_{P_{C}}\langle E\rangle, W_{P_{C}} \otimes_{R} \operatorname{gr}^{L}(N)\right)\right) \\
& =\chi^{C}\left(W / W\langle E\rangle, W \otimes_{R} \operatorname{gr}^{L}(N)\right) .
\end{aligned}
$$

By Lemmas 4.8 and 4.10 we may assume now that $N=S_{\theta}$ for some $\theta \in \Phi_{A}^{0}$ with the standard good $L_{\partial}$-filtration induced by the single generator $1 \in S_{\theta}$. Then the complex $K^{1}$ above is the Koszul complex of $E$ on $W_{P_{C}} \otimes_{R} S_{\theta}^{L}$. By BH93, Prop. 1.6.5], its homology is annihilated by both $\operatorname{gr}^{L}\left(I_{A}^{\theta}\right)=R \cdot I_{\theta}^{L}+J_{\theta} \supseteq I_{A}^{L}$ and $E$. Then equation (3.2.2) shows that $\mu_{A, i}^{L, C}\left(S_{\theta} ; \beta\right)>0$ for some $i$ implies that $C=\bar{C}_{A}^{\tau}$ is an irreducible component of $\operatorname{Spec}\left(W / W\langle E\rangle \otimes_{R} S_{A}^{L}\right)$ with $\theta \supseteq \tau \in \Phi_{A}^{L}$. By Theorem 2.14,

$$
\operatorname{codim}(W / W\langle E\rangle)+\operatorname{codim}\left(W \otimes_{R} S_{\theta}^{L}\right)=d+n-\operatorname{dim}\left(S_{\theta}\right) \geq n=\operatorname{codim}\left(\bar{C}_{A}^{\tau}\right) .
$$

So Serre's Intersection Theorem [Ser65, Ch. V, $\S$ C.1, Thm. 1] shows that $\mu_{A}^{L, \tau}\left(S_{\theta}\right)>$ 0 if and only if $\operatorname{dim}\left(S_{\theta}\right)=d$, which is to say $\theta=A$.

In the special case $L=F$, Theorem 4.11yields the holonomicity of Euler-Koszul homology of toric modules proved in [MMW05, Thm. 5.1].

In order to discuss $\operatorname{CC}^{L}\left(M_{A}(\beta)\right)$ we need more information about $\mu_{A, 0}^{L, \tau}(\beta)$ : Theorem 4.11 does not state that $\mu_{A, 0}^{L, \tau}(\beta)>0$. We show next that for generic $\beta$ this inequality does indeed hold. In Subsections 4.4 and 4.6 we will show that the genericity assumption is not necessary and in fact identify a global combinatorial lower bound for $\mu_{A, 0}^{L, \tau}(\beta)$, similar to classical statements about the holonomic rank of $M_{A}(\beta)$ in [GZK89].

Corollary 4.12. For generic (more precisely, not rank-jumping) $\beta \in \mathbb{C}^{d}$

$$
\mu_{A, 0}^{L, \tau}(\beta)=\mu_{A}^{L, \tau}=\chi^{\tau}\left(W / W\langle E\rangle, W \otimes_{R} S_{A}^{L}\right)>0
$$

for all $\tau \in \Phi_{A}^{L}$. In particular, $\mathrm{Ch}^{L}\left(M_{A}(\beta)\right)=\bigcup_{\tau \in \Phi_{A}^{L}} \bar{C}_{A}^{\tau}$.

Proof. By assumption on $\beta$ and [MMW05, Thm. 6.6], the Euler-Koszul complex $K_{A, \bullet}\left(S_{A} ; \beta\right)$ is a resolution of $M_{A}(\beta)$. Hence $\operatorname{CC}^{L}\left(K_{A, \bullet}\left(S_{A} ; \beta\right)\right)=\operatorname{CC}^{L}\left(M_{A}(\beta)\right)$ and $\mu_{A, 0}^{L, \tau}(\beta)=\mu_{A}^{L, \tau}>0$ by Theorem 4.11. So the last claim follows from Proposition 3.8 .

Recall that $\tilde{S}_{A}$ is the normalization of $S_{A}$. In Section 4.4, we show that $\mu_{A, 0}^{L, \tau}(\beta)>$ 0 for all $\beta$. This is done by reducing to $\tilde{S}_{A}$ and applying the following statement.

Corollary 4.13. For all $\tau \in \Phi_{A}^{L}$,

$$
\mu_{A, 0}^{L, \tau}\left(\tilde{S}_{A} ; \beta\right)=\mu_{A}^{L, \tau}\left(\tilde{S}_{A}\right)=\mu_{A}^{L, \tau}=\chi^{\tau}\left(W / W\langle E\rangle, W \otimes_{R} S_{A}^{L}\right)>0 .
$$

Proof. Since $\tilde{S}_{A}$ is a Cohen-Macaulay module of full dimension over $S_{A}$, its EulerKoszul complex is a resolution of $H_{A, 0}\left(\tilde{S}_{A} ; \beta\right)$ by [MMW05, Thm. 6.6]. Hence $\mu_{A, i}^{L, \tau}\left(\tilde{S}_{A} ; \beta\right)=0$ for all $i>0$ which proves the first equality. There is a short exact sequence

$$
0 \longrightarrow S_{A} \longrightarrow \tilde{S}_{A} \longrightarrow Q \longrightarrow 0
$$


where $\operatorname{dim}(Q)<d$ and the rest of the claim follows from Theorem 4.11 and Corollary 4.12

4.4. Rigidity of Euler-Koszul multiplicities. In this section we show in Theorem 4.16 that the strict positivity of $\mu_{A, 0}^{L, \tau}(\beta)$ in Corollary 4.12 holds without the genericity assumption. As a consequence, we obtain a complete description of the $L$-characteristic variety and the slopes of the $A$-hypergeometric system along coordinate varieties at the origin.

Definition 4.14. Let $\tau$ be a subset of the column set of $A$. We set $W_{\tau}=\mathbb{C}\left[x_{\tau}, \partial_{\tau}\right]$ so that $W / W I_{A}^{\tau}=\mathbb{C}\left[x_{\bar{\tau}}\right] \otimes_{\mathbb{C}}\left(W_{\tau} \otimes_{R_{\tau}} S_{\tau}\right)$.

Let $g \in \mathrm{Gl}(\mathbb{Z}, d)$ and put $A^{\prime}=g A$ and $\beta^{\prime}=g \beta$. Then note that $S_{A}=$ $S_{A^{\prime}}$ and $E^{\prime}-\beta^{\prime}=g(E-\beta)$. Hence, the Euler-Koszul complexes $K_{A, \bullet}\left(S_{A} ; \beta\right)$ and $K_{A^{\prime}, \bullet}\left(S_{A^{\prime}} ; \beta^{\prime}\right)$ are homotopy equivalent. In particular, when investigating homotopy-invariant properties of $K_{A, \bullet}\left(S_{A} ; \beta\right)$ one may replace $A$ by $g A$.

Lemma 4.15. Let $\theta \in \Phi_{A}^{0}$ and assume that the top $\operatorname{dim}(\theta)$ rows $A^{\prime}$ of $\theta$ are a lattice basis for the $\mathbb{Z}$-row span of $\theta$. Then, for all $\tau \in \Phi_{A}^{L}$,

$$
\mu_{A, i}^{L, \tau}\left(S_{\theta} ; \beta\right)= \begin{cases}\mu_{A^{\prime}, i}^{L^{\prime}, \tau^{\prime}}\left(S_{\theta^{\prime}} ; \beta^{\prime}\right) & \text { if } \tau \subseteq \theta ; \\ 0 & \text { if } \tau \nsubseteq \theta ;\end{cases}
$$

where $L^{\prime}=\left(L_{x_{A^{\prime}}}, L_{\partial_{A^{\prime}}}\right), \tau=\tau^{\prime}, \theta^{\prime}=A^{\prime}$, and $\beta^{\prime}:=\left(\beta_{1}, \ldots, \beta_{\operatorname{dim}(\theta)}\right)$.

Proof. By [MMW05, Lem. 4.8], $H_{A, i}\left(S_{\theta} ; \beta\right)=\mathbb{C}\left[x_{\bar{\theta}}\right] \otimes_{\mathbb{C}} H_{A^{\prime}, i}\left(S_{\theta^{\prime}} ; \beta^{\prime}\right)$ and hence

$$
\operatorname{gr}^{L}\left(H_{A, i}\left(S_{\theta} ; \beta\right)\right)=\mathbb{C}\left[x_{\bar{\theta}}\right] \otimes_{\mathbb{C}} \operatorname{gr}^{L^{\prime}}\left(H_{A^{\prime}, i}\left(S_{\theta^{\prime}} ; \beta^{\prime}\right)\right) \text {. }
$$

For $j \in \tau \backslash \theta, \partial_{j}$ is both annihilator and unit on $\operatorname{gr}^{L}\left(H_{A, i}\left(S_{\theta} ; \beta\right)\right)_{P_{\tau}}$ and hence $\tau \subseteq \theta$ if $\mu_{A, i}^{L, \tau}\left(S_{\theta} ; \beta\right) \neq 0$. Assume now that $\tau \subseteq \theta$. Then

$$
W_{P_{\tau}} \otimes_{W} \operatorname{gr}^{L}\left(H_{A, i}\left(S_{\theta} ; \beta\right)\right)=\mathbb{C}\left(x_{\bar{\theta}}\right) \otimes_{\mathbb{C}}\left(\left(W_{A^{\prime}}\right)_{P_{\tau}} \otimes_{W_{A^{\prime}}} \operatorname{gr}^{L^{\prime}}\left(H_{A^{\prime}, i}\left(S_{\theta^{\prime}} ; \beta^{\prime}\right)\right)\right)
$$

where the symbol $P_{\tau}$ is used for the two ideals in $W_{A}$ and $W_{A^{\prime}}$ from Definition 3.7 induced by $\tau \in \Phi_{A}^{L}$ and $\tau \in \Phi_{A^{\prime}}^{L^{\prime}}$. In particular, the length of $W_{P_{\tau}} \otimes_{W} \operatorname{gr}^{L}\left(H_{A, i}\left(S_{\theta} ; \beta\right)\right)$ over $W_{P_{\tau}}$ is the same as the length of $\left(W_{A^{\prime}}\right)_{P_{\tau}} \otimes_{W_{A^{\prime}}} \operatorname{gr}^{L^{\prime}}\left(H_{A^{\prime}, i}\left(S_{\theta^{\prime}} ; \beta^{\prime}\right)\right)$ over $\left(W_{A^{\prime}}\right)_{P_{\tau}}$.

Theorem 4.16. For any toric module $N$ and for every parameter $\beta \in \mathbb{C}^{d}$, if $\mu_{A, i}^{L, \tau}(N ; \beta)>0$ for some $i>0$ then $\mu_{A, 0}^{L, \tau}(N ; \beta)>0$.

Proof. Suppose there is a counterexample $(A, \beta, L, \tau, N)$ to the theorem. This means that $\mu_{A, i}^{L, \tau}(N ; \beta) \neq 0$ for some $i>0$ while $\mu_{A, 0}^{L, \tau}(N ; \beta)=0$. We choose a minimal counterexample in the sense that

1. A has minimal number of columns,

2. for a fixed such $A, \operatorname{dim}(N)$ is minimal, and

3. no quotient of $N$ is part of such a counterexample.

This last choice can be made since toric modules are Noetherian. Let $\theta \in \Phi_{A}^{\mathbf{0}}$ such that $I_{A}^{\theta}$ is an associated prime of $N$. Then there is a short exact sequence

$$
0 \longrightarrow S_{\theta}(-\mathbf{a}) \longrightarrow N \longrightarrow N^{\prime} \longrightarrow 0
$$

where $\mathbf{a} \in \mathbb{Z}^{d}$ is the degree of the image of $1 \in S_{\theta}$ within $N$. The long exact EulerKoszul homology sequence shows that $H_{A, 0}\left(N^{\prime} ; \beta\right)$ is a quotient of $H_{A, 0}(N ; \beta)$. 
Recall that by Lemma 4.1 the $L$-characteristic cycle is additive. Since by hypothesis $\mu_{A, 0}^{L, \tau}(N ; \beta)=0$, we conclude that $\mu_{A, 0}^{L, \tau}\left(N^{\prime} ; \beta\right)=0$ as well.

By assumption, $\left(A, \beta, L, \tau, N^{\prime}\right)$ is not a counterexample and so $\mu_{A, i}^{L, \tau}\left(N^{\prime} ; \beta\right)=0$ for all $i$. It follows that the $\mu_{A, i}^{L, \tau}$-vanishing patterns of $N$ and of $S_{\theta}(-\mathbf{a})$ coincide. In particular, $S_{\theta}(-\mathbf{a})$ is a counterexample. Since $\operatorname{dim}\left(S_{\theta}\right) \leq \operatorname{dim}(N)$, and since $S_{\theta}$ is a domain, $S_{\theta}(-\mathbf{a})$ is actually a minimal counterexample. Since $H_{A, i}\left(S_{\theta}(-\mathbf{a}) ; \beta\right)=$ $H_{A, i}\left(S_{\theta} ; \beta-\mathbf{a}\right)$ up to a $\mathbb{Z}^{d}$-shift we may assume that $N=S_{\theta}$. But if $\theta \neq A$ then Lemma 4.15 yields a counterexample whose matrix $A^{\prime}$ has strictly fewer columns than $A$. Therefore $\theta=A$ by minimality of $A$ and $\left(A, \beta, L, \tau, S_{A}\right)$ is a minimal counterexample.

By Corollary 4.13, $\mu_{A, 0}^{L, \tau}\left(\tilde{S}_{A} ; \beta\right)>0$ where $\tilde{S}_{A}$ is the normalization of $S_{A}$. For appropriate $\mathbf{a} \in \mathbb{Z}^{d}$, there is a short exact sequence

$$
0 \longrightarrow \tilde{S}_{A}(-\mathbf{a}) \longrightarrow S_{A} \longrightarrow Q \longrightarrow 0
$$

where $Q$ is not a counterexample as $\operatorname{dim}(Q)<d$. By additivity of the $L$-characteristic cycle, $\mu_{A, 0}^{L, \tau}\left(S_{A} ; \beta\right)=0$ implies $\mu_{A, 0}^{L, \tau}(Q ; \beta)=0$ and so, as $Q$ is not a counterexample, $\mu_{A, i}^{L, \tau}(Q ; \beta)=0$ for all $i$. This yields $\mu_{A, i}^{L, \tau}\left(S_{A} ; \beta\right)=\mu_{A, i}^{L, \tau}\left(\tilde{S}_{A} ; \beta\right)$ for all $i$ which is a contradiction for $i=0$.

Finally, from Theorem 4.16, Corollary 4.12 and Theorem 4.11, we obtain the $L$-characteristic variety of the $A$-hypergeometric system and, as a consequence, the slopes of the $A$-hypergeometric system along coordinate varieties at the origin.

Corollary 4.17. For all $\tau \in \Phi_{A}^{L}$ and $\beta \in \mathbb{C}^{d}, \mu_{A, 0}^{L, \tau}(\beta)>0$. In consequence, the $L$-characteristic variety of $M_{A}(\beta)$ consists for every $\beta$ precisely of all conormal closures of the torus orbits indexed by the faces in the $(A, L)$-umbrella:

$$
\mathrm{Ch}^{L}\left(M_{A}(\beta)\right)=\bigcup_{\tau \in \Phi_{A}^{L}} \bar{C}_{A}^{\tau} .
$$

Corollary 4.18. For $L=p F+q V$ as in (3.1.2), $L^{\prime}=p^{\prime} / q^{\prime}$ is a slope of $M_{A}(\beta)$ along $Y$ at $0 \in Y$ if and only if $\Phi_{A}^{L}$ jumps at $L=L^{\prime}$.

4.5. Euler-Koszul characteristic and volume. In this section we develop an explicit combinatorial formula for the Euler-Koszul characteristic $\mu_{A}^{L, \tau}$ for all $\tau \in$ $\Phi_{A}^{L}$, Theorem 4.21 below. For generic $\beta$, this formula determines the characteristic cycle of $M_{A}(\beta)$ as stated in Corollary 4.17 below, but in general it provides only a lower bound.

Note that $\operatorname{rk}\left(M_{A}(\beta)\right)=\mu_{A, 0}^{F, \emptyset}(\beta)$, and that $\mu_{A, 0}^{L, \tau}(\beta)=\mu_{A}^{L, \tau}$ if $\beta$ is not a rankjumping parameter by MMW05, Thm. 6.6]. It is a classical result that $\operatorname{rk}\left(M_{A}(\beta)\right)=$ $\operatorname{vol}_{\mathbb{Z}^{d}}\left(\Delta_{A}^{F}\right)$ for generic $\beta$ (see [GZK89, Ado94]). Theorem 4.21 below contains this result as a special case when $\tau=\emptyset$ and $L=F$. Our generalization of the fact that generic rank equals volume reads

$$
\mu_{A}^{L, \emptyset}=\operatorname{vol}_{\mathbb{Z}^{d}}\left(\bigcup_{\tau \in \Phi_{A}^{L, d-1}}\left(\Delta_{0}^{F} \backslash \operatorname{conv}(\tau)\right)\right) .
$$


This paragraph outlines our strategy towards Theorem 4.21, By Theorem 4.11, $\mu_{A}^{L, \tau}$ is an intersection multiplicity and hence additive. Applying $\mu_{A}^{L, \tau}$ to the composition chain (2.7.1) of $S_{A}^{L}$ yields

$$
\begin{aligned}
\mu_{A}^{L, \tau}=\chi^{\tau}\left(W /\langle E\rangle, S_{A}^{L}\right) & =\sum_{i=1}^{l} \chi^{\tau}\left(W /\langle E\rangle, S_{\tau_{i}}\right) \\
& =\sum_{\tau \subseteq \tau^{\prime} \in \Phi_{A}^{L, d-1}} \nu_{A}^{L, \tau^{\prime}} \cdot \chi^{\tau}\left(W /\langle E\rangle, S_{\tau^{\prime}}\right) \\
& =\sum_{\tau \subseteq \tau^{\prime} \in \Phi_{A}^{L, d-1}}\left[\mathbb{Z}^{d}: \mathbb{Z} \tau^{\prime}\right] \cdot \mu_{A}^{L, \tau}\left(S_{\tau^{\prime}}\right) .
\end{aligned}
$$

For the third equality note that $\left(R / I_{A}^{\tau^{\prime}}\right)_{I_{A}^{\tau}}=0$ unless $\tau \subseteq \tau^{\prime}$, and then use $\operatorname{dim}\left(S_{\tau^{\prime}}\right)=\operatorname{dim}\left(\tau^{\prime}\right)+1$ in Theorem 4.11. Proposition 2.17 gives the last equality. By equation (4.5.1) it is sufficient to know the multiplicities $\mu_{A}^{L, \tau}\left(S_{\tau^{\prime}}\right)$ for $\tau \subseteq \tau^{\prime} \in \Phi_{A}^{L, d-1}$. To determine these we first reduce to $\tau^{\prime}=A$ and then to $\tau=\emptyset$ while controlling the appropriate intersection multiplicity. Finally, we give a combinatorial description in that case. Some of our arguments are similar to GZK89, $\S \S 2.3-2.4]$ and Ado94, $\S 5]$.

Let $\Phi_{A}^{L} \ni \tau \subseteq \tau^{\prime} \in \Phi_{A}^{L, d-1}$ and abbreviate $\mu:=\mu_{A}^{L, \tau}\left(S_{\tau^{\prime}}\right)=\chi^{\tau}\left(W /\langle E\rangle, S_{\tau^{\prime}}\right)$. Since $E$ is a regular sequence in $W, \mu$ can be computed from the Koszul complex of $E$ on $W_{P_{\tau}} \otimes_{R} S_{\tau^{\prime}}$. Since $\partial_{\bar{\tau}^{\prime}}$ is zero on $S_{\tau^{\prime}}$ one can erase the $\partial_{\bar{\tau}}$ terms in $E$. Now the (canonical basis of the) ambient lattice $\mathbb{Z}^{d}=\mathbb{Z} A$ can be replaced by (a lattice basis of) $\mathbb{Z} \tau^{\prime}$; this leaves the quantities $W,\langle E\rangle, I_{\tau}$ and $S_{\tau^{\prime}}$ invariant. We have thus reduced the problem to the case $\tau^{\prime}=A$.

We now reduce to the case $\tau=\emptyset$. By the Fourier transform we may use $\partial$ as base variables and identify $-x_{j} \in W$ with the (symbol of the) partial derivation along $\partial_{j}$. Since $\chi^{\tau}$ is determined at a generic point of $C_{A}^{\tau}$, we may replace $W$ by $W^{\prime}=W\left[\partial_{\tau}^{-1}\right]$ whose spectrum $T^{*} U$ is the cotangent space of $U=\operatorname{Spec}\left(R\left[\partial_{\tau}^{-1}\right]\right)$.

We now modify the pair $\left(\tau^{\prime}, \tau\right)$ into a more convenient one, while keeping track of $\mu$. A row operation $\left(\hat{\tau}^{\prime}, \hat{\tau}\right)=g\left(\tau^{\prime}, \tau\right)$ defined by $g \in \mathrm{Gl}(\mathbb{Z}, d)$ amounts to a basis change in the $d$-torus $\mathbb{T}$, so $\mu=\mu_{\hat{\tau}^{\prime}}^{L, \hat{\tau}}\left(S_{\hat{\tau}^{\prime}}\right)$. On the other hand, for a triple $j \in \tau^{\prime}$, $i \in \tau, m \in \mathbb{Z}$, the elementary column operation $\hat{\mathbf{a}}_{j}=\mathbf{a}_{j}+m \mathbf{a}_{i}$ transforming $\tau^{\prime}$ into $\hat{\tau}^{\prime}$ corresponds to the automorphism $\hat{\partial}_{j}=\partial_{j} \partial_{i}^{m}$ of $U$. The induced map on $W^{\prime}$ obviously transforms $I_{\tau^{\prime}}$ into $I_{\hat{\tau}^{\prime}}$. Note that $x_{i} \partial_{i}=\hat{x}_{i} \hat{\partial}_{i}+m \hat{x}_{j} \hat{\partial}_{j}$ and $x_{k} \partial_{k}=\hat{x}_{k} \hat{\partial}_{k}$ for $k \neq i$. Thus, $\mu=\mu_{\hat{\tau}^{\prime}}^{L, \hat{\tau}}\left(S_{\hat{\tau}^{\prime}}\right)$. In other words, all elementary column operations $\mathbf{a}_{j} \mapsto \mathbf{a}_{j}+m \mathbf{a}_{i}$ with $i \in \tau$, all column switches $\mathbf{a}_{i} \leftrightarrow \mathbf{a}_{j}$ with $i, j \in \tau$ or $i, j \notin \tau$, and all $\mathbb{Z}$-invertible row operations leave $\mu$ invariant.

After a suitable sequence of such transformations, we may assume that

$$
\tau=\left(\begin{array}{ll}
I & 0 \\
0 & 0
\end{array}\right), \quad I=\left(\begin{array}{ccc}
k_{1} & & 0 \\
& \ddots & \\
0 & & k_{e}
\end{array}\right)
$$

where $k_{i} \in \mathbb{N} \backslash\{0\}$. Note that the product $k_{1} \cdots k_{e}$ is the index $\left[\left(\mathbb{Z} \tau^{\prime} \cap \mathbb{Q} \tau\right): \mathbb{Z} \tau\right]$ of $\mathbb{Z} \tau$ in $\mathbb{Z} \tau^{\prime} \cap \mathbb{Q} \tau$.

We next consider the $k_{1}$-fold covering space $\kappa_{1}: U \rightarrow U$ induced by $\hat{\partial}_{1}^{k_{1}}=\partial_{1}$. Let $\left(\hat{\tau}, \hat{\tau}^{\prime}\right)$ be the matrices obtained from $\left(\tau, \tau^{\prime}\right)$ by dividing the first column by $k_{1}$ 
and let $\hat{E}$ be the Euler vector fields of $\hat{\tau}$. As one checks, $\hat{x}_{1} \hat{\partial}_{1}=k_{1} x_{1} \partial_{1}$ and so $\kappa_{1}(\operatorname{Var}(\hat{E}))=\operatorname{Var}(E), \kappa_{1}\left(\operatorname{Var}\left(I_{\hat{\tau}^{\prime}}\right)\right)=\operatorname{Var}\left(I_{\tau^{\prime}}\right)$, and $\kappa_{1}\left(C_{A}^{\hat{\tau}}\right)=C_{A}^{\tau}$. Moreover, the degree of $\kappa_{1}$ is equal to $k_{1}$ on $C_{A}^{\tau}$, and equal to 1 on both $\operatorname{Var}(E)$ and $\operatorname{Var}\left(I_{\tau^{\prime}}\right)$. By Example 8.2.5 in [Ful98,

$$
\mu=k_{1} \cdot \chi^{\hat{\tau}}\left(W /\langle\hat{E}\rangle, S_{\hat{\tau}^{\prime}}\right) .
$$

Analogous transformations for $k_{2}, \ldots, k_{e}$ followed by suitable elementary column operations as above yield

$$
\hat{\tau}=\left(\begin{array}{cc}
I & 0 \\
0 & 0
\end{array}\right), \quad \hat{\tau}^{\prime}=\left(\begin{array}{ccc}
I & 0 & 0 \\
0 & 0 & \check{\tau}
\end{array}\right), \quad I=\left(\begin{array}{lll}
1 & & 0 \\
& \ddots & \\
0 & & 1
\end{array}\right)
$$

where now

$$
\mu=k_{1} \cdots k_{e} \cdot \chi^{\hat{\tau}}\left(W / E_{\hat{\tau}^{\prime}}, S_{\hat{\tau}^{\prime}}\right)=\left[\left(\mathbb{Z} \tau^{\prime} \cap \mathbb{Q} \tau\right): \mathbb{Z} \tau\right] \cdot \chi^{\hat{\tau}}\left(W / E_{\hat{\tau}^{\prime}}, S_{\hat{\tau}^{\prime}}\right) .
$$

Note that $\check{\tau}$ is pointed since $\tau$ is a face of $\tau^{\prime}$. Let $\check{E}$ be the Euler vector fields of $\check{\tau}$. Then on $T^{*} U,\langle E\rangle=\left\langle\hat{x}_{1} \hat{\partial}_{1}, \ldots, \hat{x}_{e} \hat{\partial}_{e}, \check{E}\right\rangle=\left\langle\hat{x}_{1}, \ldots, \hat{x}_{e}, \check{E}\right\rangle$ as well as $I_{\hat{\tau}^{\prime}}=$ $\left\langle\hat{\partial}_{e+1}, \ldots, \hat{\partial}_{|\tau|}\right\rangle+I_{\tilde{\tau}}$. This establishes the announced reduction to the case $\tau=\emptyset$,

$$
\mu=\left[\left(\mathbb{Z} \tau^{\prime} \cap \mathbb{Q} \tau\right): \mathbb{Z} \tau\right] \cdot \chi^{\emptyset}\left(W /\langle\check{E}\rangle, S_{\check{\tau}}\right)=\left[\left(\mathbb{Z} \tau^{\prime} \cap \mathbb{Q} \tau\right): \mathbb{Z} \tau\right] \cdot \mu_{\check{\tau}}^{L, \emptyset}\left(S_{\check{\tau}}\right) .
$$

The constructed lattice $\mathbb{Z} \check{\tau} \subseteq \mathbb{Z} \tau^{\prime} \subseteq \mathbb{Z}^{d}$ corresponds to a splitting of the natural projection

$$
\pi_{\tau, \tau^{\prime}}: \mathbb{Z} \tau^{\prime} \longrightarrow \mathbb{Z} \tau^{\prime} /\left(\mathbb{Z} \tau^{\prime} \cap \mathbb{Q} \tau\right)
$$

which identifies the positive semigroups $\mathbb{N} \check{\tau}$ and $\pi_{\tau, \tau^{\prime}}\left(\mathbb{N} \tau^{\prime}\right)$.

Definition 4.19. In a lattice $\Lambda$, the volume function $\operatorname{vol}_{\Lambda}$ is normalized so that the unit simplex of $\Lambda$ has volume 1 . We abbreviate $\operatorname{vol}_{\tau, \tau^{\prime}}:=\operatorname{vol}_{\pi_{\tau, \tau^{\prime}}\left(\mathbb{Z} \tau^{\prime}\right)}$.

We continue under the assumption that $\tau=\emptyset, \check{\tau}=\tau^{\prime}=A$, and compute the intersection multiplicity of $\operatorname{Var}(E)$ with $\operatorname{Var}\left(I_{A}\right)$ along $C_{A}^{\emptyset}=X$ inside $T^{*} U=$ $\operatorname{Spec}\left(W^{\prime}\right)$,

$$
\check{\mu}:=\mu_{\check{\tau}}^{L, \emptyset}\left(S_{\check{\tau}}\right)=\chi^{\emptyset}\left(W /\langle\check{E}\rangle, S_{\check{\tau}}\right) .
$$

Since for $y \in\left(\mathbb{C}^{*}\right)^{|\check{\tau}|}$ the linear polynomials $x_{1}-y_{1}, \ldots, x_{|\check{\tau}|}-y_{|\check{\tau}|}$ form a regular sequence on $W /\langle E\rangle$, on $S_{\check{\tau}}$ and on $W / P_{\emptyset}$, we may replace $W$ by $R, E_{i}$ by $\bar{E}_{i}:=$ $\sum_{j} a_{i, j} y_{j} \partial_{j}$, and $C_{A}^{\emptyset}$ by $\{0\}=\operatorname{Var}\left(\partial_{\tilde{\tau}}\right)$. By the sequence (4.3.1), we may further replace $S_{\check{\tau}}$ by its normalization $\tilde{S}_{\tilde{\tau}}$. Thus, we have reduced to computing

$$
\check{\mu}=\chi^{\{0\}}\left(R /\langle\bar{E}\rangle, \tilde{S}_{\check{\tau}}\right) .
$$

For generic $y$, the function on $\operatorname{Spec}\left(\tilde{S}_{\tilde{\tau}}\right)$

$$
f:=\sum_{j=1}^{|\check{\tau}|} y_{j} \partial_{j}=\sum_{j=1}^{|\check{\tau}|} y_{j} t^{\mathbf{a}_{j}}
$$

is by Kou76, Thm. 6.1] and its proof Newton nondegenerate at the origin. We can interpret $\bar{E}$ as functions on $\operatorname{Spec}\left(\tilde{S}_{\check{\tau}}\right)$,

$$
\bar{E}_{i}=t_{i} \frac{\partial f}{\partial t_{i}}
$$


Let $\mathfrak{m}_{\check{\tau}} \subseteq S_{\check{\tau}}$ and $\tilde{\mathfrak{m}}_{\check{\tau}} \subseteq \tilde{S}_{\check{\tau}}$ be the maximal ideals at 0 . In the special case where $\check{\tau}$ is the unit matrix, and where hence $\tilde{S}_{\tilde{\tau}}$ is a polynomial ring, [Kou76, Thm. 2.8] states that the Koszul complex of $\bar{E}$ on $\tilde{S}_{\check{\tau}}$ is acyclic in positive dimension when completed at $\tilde{\mathfrak{m}}_{\tilde{\tau}}$. The $\mathfrak{m}_{\tilde{\tau}^{-}}$and $\tilde{\mathfrak{m}}_{\tilde{\tau}^{-}}$-adic topologies on $\tilde{S}_{\tilde{\tau}}$ are equivalent and completion at $\tilde{\mathfrak{m}}_{\tilde{\tau}}$ can be replaced by completion at $\mathfrak{m}_{\tilde{\tau}}$. By faithful flatness of completion this implies that the Koszul complex of $\bar{E}$ on $\left(\tilde{S}_{\check{\tau}}\right)_{\mathfrak{m}_{\tilde{\tau}}}$ is acyclic in positive dimension and hence

$$
\check{\mu}=\operatorname{dim}_{\mathbb{C}}\left(\left(\tilde{S}_{\check{\tau}}\right)_{\mathfrak{m}_{\check{\tau}}} /\langle\bar{E}\rangle\right) \text {. }
$$

Since this dimension is finite, localization can be replaced by completion at $\mathfrak{m}_{\tilde{\tau}}$ or equivalently at $\tilde{\mathfrak{m}}_{\tilde{\tau}}$. Then [Kou76, Thm. A.I] states that this dimension equals

$$
\check{\mu}=\operatorname{vol}_{\mathbb{Z} \check{\tau}}\left(\Gamma_{-}(f)\right), \quad \Gamma_{-}(f)=\operatorname{conv}(\check{\tau} \cup\{0\}) \backslash \operatorname{conv}(\check{\tau}) .
$$

One checks that Kouchnirenko's result applies to a general normal semigroup ring $\tilde{S}_{\breve{\tau}}$ for which $\check{\tau}$ is pointed (see [Kou76, $\left.\S 2.12\right]$ ). The following definition serves to interpret this volume in terms of the original matrix $A$, and mirrors the one given in GZK89, §2.1].

Definition 4.20. For $\Phi_{A}^{L} \ni \tau \subseteq \tau^{\prime} \in \Phi_{A}^{L, d-1}$, define the polyhedra

$$
P_{\tau, \tau^{\prime}}:=\operatorname{conv}\left(\pi_{\tau, \tau^{\prime}}\left(\tau^{\prime} \cup\{0\}\right)\right), \quad Q_{\tau, \tau^{\prime}}:=\operatorname{conv}\left(\pi_{\tau, \tau^{\prime}}\left(\tau^{\prime} \backslash \tau\right)\right) .
$$

We are now ready to give the promised multiplicity formula.

Theorem 4.21. For all $\tau \in \Phi_{A}^{L}$, the multiplicity of $\bar{C}_{A}^{\tau}$ in $\operatorname{CC}^{L}\left(K_{A, \bullet}\left(S_{A} ; \beta\right)\right)$ is

$$
\mu_{A}^{L, \tau}=\sum_{\tau \subseteq \tau^{\prime} \in \Phi_{A}^{L, d-1}}\left[\mathbb{Z}^{d}: \mathbb{Z} \tau^{\prime}\right] \cdot\left[\left(\mathbb{Z} \tau^{\prime} \cap \mathbb{Q} \tau\right): \mathbb{Z} \tau\right] \cdot \operatorname{vol}_{\tau, \tau^{\prime}}\left(P_{\tau, \tau^{\prime}} \backslash Q_{\tau, \tau^{\prime}}\right) .
$$

Corollary 4.22. If $\tau=\emptyset$, then the local degree of $S_{A}^{L}$ at the origin equals

$$
\mu_{A}^{L, \emptyset}=\operatorname{vol}_{\mathbb{Z}^{d}}\left(\bigcup_{\tau^{\prime} \in \Phi_{A}^{L, d-1}}\left(\Delta_{\tau^{\prime}}^{F} \backslash \operatorname{conv}\left(\tau^{\prime}\right)\right)\right) \leq \operatorname{vol}_{\mathbb{Z}^{d}}\left(\Delta_{A}^{F}\right) .
$$

Proof. Let $\tau^{\prime} \in \Phi_{A}^{L, d-1}$. For $\tau=\emptyset, \operatorname{vol}_{\tau, \tau^{\prime}}=\operatorname{vol}_{\mathbb{Z} \tau^{\prime}}, P_{\tau, \tau^{\prime}}=\Delta_{\tau^{\prime}}^{F}$, and $Q_{\tau, \tau^{\prime}}=$ $\operatorname{conv}\left(\tau^{\prime}\right)$. Clearly, $\left[\left(\mathbb{Q} \tau \cap \mathbb{Z} \tau^{\prime}\right): \mathbb{Z} \tau\right]=1$ and $\operatorname{vol}_{\mathbb{Z} \tau^{\prime}}\left(\Delta_{\tau^{\prime}}^{F}\right)=\operatorname{vol}_{\mathbb{Z}^{d}}\left(\Delta_{\tau^{\prime}}^{F}\right) /\left[\mathbb{Z}^{d}: \mathbb{Z} \tau^{\prime}\right]$. Then Theorem 4.21 implies the equality while the inequality is obvious.

Remark 4.23. If all facets $\tau^{\prime}$ of the $(A, L)$-umbrella are $F$-homogeneous then the volumes $\operatorname{vol}_{\mathbb{Z}^{d}}\left(\operatorname{conv}\left(\tau^{\prime}\right)\right)$ are zero and one obtains

$$
\mu_{A}^{L, \emptyset}=\operatorname{vol}_{\mathbb{Z}^{d}}\left(\bigcup_{\tau^{\prime} \in \Phi_{A}^{L, d-1}} \Delta_{\tau^{\prime}}^{F}\right) .
$$

In particular, $\mu_{A}^{F, \emptyset}=\operatorname{vol}_{\mathbb{Z}^{d}}\left(\Delta_{A}^{F}\right)$.

Example 4.24. We continue Example 3.12 and investigate characteristic cycles. The following table, whose rows are indexed by the three weight vectors $L=F+t V$ considered in Example 3.12, lists in its columns the nonzero multiplicities $\mu_{A, 0}^{L, \tau}(\beta)$ for generic $\beta$. Genericity implies that $\mu_{A, i}^{L, \tau}(\beta)=0$ for $i>0$, so that $\mu_{A, 0}^{L, \tau}(\beta)=\mu_{A}^{L, \tau}$.

The matrix $A$ permits two rank-jumping parameters, $\mathcal{E}(A)=\{(2,1),(3,1)\}$. In both cases, $H_{A, 1}\left(S_{A} ; \beta\right) \cong D /\left\langle\partial_{1}, \ldots, \partial_{n}\right\rangle$ while $H_{A, 2}\left(S_{A} ; \beta\right) \cong 0$. As $\langle\partial\rangle=P_{\emptyset}$, 
FIgURE 3. Euler-Koszul multiplicities in Example 4.24

\begin{tabular}{|c|c|c|c|c|c|c|c|c|c|c|}
\hline$t$ & $\emptyset$ & $\{1\}$ & $\{2\}$ & $\{3\}$ & $\{4\}$ & $\{1,3\}$ & $\{1,4\}$ & $\{2,3\}$ & $\{2,4\}$ & $\{3,4\}$ \\
\hline 0 & 13 & 12 & 1 & & 13 & & 12 & & 1 & \\
\hline 1 & 11 & 3 & 1 & 10 & 8 & 3 & & & 1 & 7 \\
\hline 4 & 5 & 3 & 2 & 5 & & 3 & & 2 & & \\
\hline
\end{tabular}

with $\beta \in \mathcal{E}(A)$ one has $\mu_{A, 1}^{L, \tau}(\beta)=1$ if $\tau=\emptyset$, and $\mu_{A, 1}^{L, \tau}(\beta)=0$ otherwise. (This holds for all $L \in \mathbb{Q}^{2 n}$ as long as $L_{x}+L_{\partial}>0$ since $\operatorname{gr}^{L}\left(\left\langle\partial_{1}, \ldots, \partial_{n}\right\rangle\right)$ is independent of $L)$. In accordance with Corollary 4.13, the multiplicities $\mu_{A, 0}^{L, \tau}(\beta)$ for $\beta \in \mathcal{E}(A)$ are given by the data in the table above, with $\mu_{A, 0}^{L, \emptyset}(\beta)$ incremented by one. This behavior is typical in dimension two as the following proposition shows.

Proposition 4.25. If $d=2$ then for every rank-jumping $\beta$ and for all $L$ with $L_{x}+L_{\partial}>0$ one has $\mu_{A, 1}^{L, \tau}(\beta)=1$ if $\tau=\emptyset$ and $\mu_{A, 1}^{L, \tau}(\beta)=0$ otherwise.

Proof. Recall that $\tilde{S}_{A}$ is the Cohen-Macaulay $S_{A^{-} \text {-module }} \bigoplus_{\mathbf{a} \in \mathbb{Q}_{+} A \cap \mathbb{Z}^{d}} t^{\mathbf{a}}$. We first construct the minimal toric submodule of $\tilde{S}_{A}$ that contains $S_{A}$ and satisfies Serre's condition $S_{2}$. To that end, apply the local cohomology functor $H_{\mathfrak{m}}^{\bullet}(-)$ to the sequence (4.3.1); we obtain $H_{\mathfrak{m}}^{0}(Q)=H_{\mathfrak{m}}^{1}\left(S_{A}\right)$. Let $C$ be the (toric) preimage of $H_{\mathfrak{m}}^{0}(Q)$ under the projection $\tilde{S}_{A} \rightarrow Q$; as $C \subseteq \tilde{S}_{A}$ we have $H_{\mathfrak{m}}^{0}(C)=0$. Applying $H_{\mathfrak{m}}^{\bullet}(-)$ to

$$
0 \longrightarrow S_{A} \longrightarrow C \longrightarrow H_{\mathfrak{m}}^{0}(Q) \longrightarrow 0
$$

we find an exact piece $0 \rightarrow H_{\mathfrak{m}}^{0}\left(H_{\mathfrak{m}}^{0}(Q)\right) \rightarrow H_{\mathfrak{m}}^{1}\left(S_{A}\right) \rightarrow H_{\mathfrak{m}}^{1}(C) \rightarrow H_{\mathfrak{m}}^{1}\left(H_{\mathfrak{m}}^{0}(Q)\right)=0$. As the second arrow is an isomorphism by construction, the two-dimensional module $C$ has depth two and is hence Cohen-Macaulay.

Application of the Euler-Koszul functor to the short exact sequence (4.5.2) shows that $H_{A, 1}\left(S_{A} ; \beta\right) \cong H_{A, 2}\left(H_{\mathfrak{m}}^{0}(Q) ; \beta\right)$. Since $H_{\mathfrak{m}}^{0}(Q)$ is a toric Artinian quotient of $C$, it has a toric filtration whose quotients are of the form $R /\left\langle\partial_{1}, \ldots, \partial_{n}\right\rangle \cdot t^{\beta}$ where $\beta \in \mathcal{E}(A)$ and each such $\beta$ occurs exactly once (see [MMW05, Thm. 6.6, Thm .9.1]). Hence, $H_{A, 1}\left(S_{A} ; \beta\right) \cong D /\left\langle\partial_{1}, \ldots, \partial_{n}\right\rangle$ if $\beta \in \mathcal{E}(A)$ and zero otherwise. It follows as in the example above that $\operatorname{gr}^{L}\left(H_{A, 1}\left(S_{A} ; \beta\right)\right) \cong W /\left\langle\partial_{1}, \ldots, \partial_{n}\right\rangle$ in the nonvanishing case.

Remark 4.26. (1) The module $C$ constructed in the above proof is actually a ring, the ideal transform of $S_{A}$ relative to $\mathfrak{m}$ from [BS98, Thm. 2.2.4].

(2) It is suggestive, particularly in the light of Example 4.24, to view $\mu_{A}^{L, \emptyset}$ as " $L$-rank". Specifically, one might speculate whether the $L$-rank detects rank jumps in the sense that $\mu_{A, i}^{L, \emptyset}(\beta)=0$ for all $i>0$ implies that $\beta$ is not rank-jumping. We think that this is plausible.

4.6. Generic Euler-Koszul homology. In [GZK89], I.M. Gel'fand, M.M. Kapranov and A.V. Zelevinskiur proved that in the projective case the rank of $M_{A}(\beta)$ is always at least equal to $\operatorname{vol}_{\mathbb{Z}^{d}}\left(\Delta_{A}^{F}\right)=\mu_{A}^{F, \emptyset}$, cf. Remark 4.23, this was generalized by A. Adolphson in Ado94. In this section, we prove that every Euler-Koszul multiplicity $\mu_{A, 0}^{L, \tau}(\beta)$ is bounded from below by the Euler-Koszul characteristic $\mu_{A}^{L, \tau}$ for all $\beta$. This yields some evidence for the following conjecture. 
Conjecture 4.27. For fixed $A, L$ and $\tau, \mu_{A, 0}^{L, \tau}(\beta)$ is upper semicontinuous in $\beta$.

A proof of this would generalize [MMW05, Thm. 2.6, Thm. 7.5] which correspond to $\tau=\emptyset$ and $L=F$. However, we do not know how to approach this question since it involves a flat deformation in combination with a specialization. The case where $\tau=\emptyset$ and $L=F$ is much easier since there one may skip the computation of the graded by the Cauchy-Kovalevskaya-Kashiwara Theorem (see [SST00, Thm. 1.4.14, Thm. 1.4.19]).

Theorem 4.28. For any $\tau \in \Phi_{A}^{L}$ and $\beta \in \mathbb{C}^{d}, \mu_{A, 0}^{L, \tau}(\beta) \geq \mu_{A}^{L, \tau}$. Equality holds if $\beta$ is generic (more precisely, not rank-jumping).

Proof. The sequence (4.3.1) yields a four-step exact sequence

$$
0 \longrightarrow H_{A, 1}(Q ; \beta) \longrightarrow H_{A, 0}\left(S_{A} ; \beta\right) \longrightarrow H_{A, 0}\left(\tilde{S}_{A} ; \beta\right) \longrightarrow H_{A, 0}(Q ; \beta) \longrightarrow 0 \text {. }
$$

By Corollary 4.13 and Lemma 4.29 below,

$$
\mu_{A, 0}^{L, \tau}\left(S_{A} ; \beta\right) \geq \mu_{A, 0}^{L, \tau}\left(\tilde{S}_{A} ; \beta\right)=\mu_{A}^{L, \tau} .
$$

The second claim is part of Corollary 4.12

Lemma 4.29. For any toric module $N$ with $\operatorname{dim}(N)<d, \tau \in \Phi_{A}^{L}$, and $\beta \in \mathbb{C}^{d}$,

$$
\mu_{A, 0}^{L, \tau}(N ; \beta) \leq \mu_{A, 1}^{L, \tau}(N ; \beta) .
$$

Proof. Let $\left(A^{\prime}, \beta^{\prime}\right)=(g A, g \beta)$ for some $g \in \mathrm{Gl}(\mathbb{Z}, d)$. Then the Euler-Koszul complexes $K_{A, \bullet}(N ; \beta)$ and $K_{A^{\prime}, \bullet}\left(N ; \beta^{\prime}\right)$ are homotopic for any toric module $N$. Hence we may replace $(A, \beta)$ by $\left(A^{\prime}, \beta^{\prime}\right)$ and choose $g$ generic. Fix the toric module $N$ of dimension less than $d$. Then the Koszul complex $K_{A, \bullet}\left(N ; \beta^{\prime}\right)$ of the endomorphisms $E^{\prime}-\beta^{\prime}:=\left(E_{1}-\beta_{1}, \ldots, E_{d-1}-\beta_{d-1}\right)$ on the $\mathbb{Z}^{d}$-graded left $D$-module $D \otimes_{R} N$, defined as in Definition 4.6, is homologically $L$-holonomic. To see this it is by the long Euler-Koszul homology sequence enough to consider a length-one toric module $N=S_{\theta}$ for some $\theta \in \Phi_{A}^{0}$. Then, using Proposition 3.8, genericity of $g$ assures that $W\left\langle I_{\theta}^{L}, E^{\prime}\right\rangle$ is an $n$-dimensional ideal and hence $\operatorname{dim} \mathrm{Ch}^{L}\left(H_{A, i}\left(N ; \beta^{\prime}\right)\right) \leq n$ by the spectral sequence

$$
H_{i}\left(\operatorname{gr}^{L}\left(K_{A, \bullet}\left(N ; \beta^{\prime}\right)\right)\right) \Longrightarrow \operatorname{gr}^{L}\left(H_{A, i}\left(N ; \beta^{\prime}\right)\right) .
$$

We interpret $K_{A, \bullet}(N ; \beta)$ as the complex induced by $E_{d}-\beta_{d}$ on $K_{A, \bullet}\left(N ; \beta^{\prime}\right)$ and abbreviate $H_{i}:=H_{A, i}(N ; \beta)$ and $H_{i}^{\prime}:=H_{A, i}\left(N ; \beta^{\prime}\right)$. There is a double complex spectral sequence abutting to $H_{\bullet}$ with $E_{1}$-term $H_{\bullet}^{\prime}$ and differential $d_{1}$ induced by $E_{d}-\beta_{d}$ which collapses at the $E_{2}$-term. In particular, $H_{0}=H_{0}^{\prime} /\left(E_{d}-\beta_{d}\right) H_{0}^{\prime}$ and there is a short exact sequence of holonomic $D$-modules

$$
0 \longrightarrow \operatorname{ker}_{H_{0}^{\prime}}\left(E_{d}-\beta_{d}\right) \longrightarrow H_{1} \rightarrow H_{1}^{\prime} /\left(E_{d}-\beta_{d}\right) H_{1}^{\prime} \longrightarrow 0 \text {. }
$$

Using the exact sequence

$$
0 \longrightarrow \operatorname{ker}_{H_{0}^{\prime}}\left(E_{d}-\beta_{d}\right) \longrightarrow H_{0}^{\prime} \stackrel{E_{d}-\beta_{d}}{\longrightarrow} H_{0}^{\prime} \longrightarrow H_{0}^{\prime} /\left(E_{d}-\beta_{d}\right) H_{0}^{\prime} \longrightarrow 0
$$

it follows that

$$
\begin{aligned}
\mathrm{CC}^{L}\left(H_{0}\right) & =\mathrm{CC}^{L}\left(H_{0}^{\prime} /\left(E_{d}-\beta_{d}\right) H_{0}^{\prime}\right) \\
& =\mathrm{CC}^{L}\left(\operatorname{ker}_{H_{0}^{\prime}}\left(E_{d}-\beta_{d}\right)\right) \\
& =\mathrm{CC}^{L}\left(H_{1}\right)-\mathrm{CC}^{L}\left(H_{1}^{\prime} /\left(E_{d}-\beta_{d}\right) H_{1}^{\prime}\right) .
\end{aligned}
$$


We conclude that $\mathrm{CC}^{L}\left(H_{1}\right)-\mathrm{CC}^{L}\left(H_{0}\right)=\mathrm{CC}^{L}\left(H_{1}^{\prime} /\left(E_{d}-\beta_{d}\right) H_{1}^{\prime}\right)$ is nonnegative which implies the claim.

\section{Projectivized hypergeometric Systems}

Here we introduce and study a natural extension of the $A$-hypergeometric system $M_{A}(\beta)$ on $X=\mathbb{C}^{n}$ to a sheaf on $\left(\mathbb{P}_{\mathbb{C}}^{1}\right)^{n}$ where $\mathbb{P}_{\mathbb{C}}^{1}=\mathbb{C} \sqcup\{\infty\}$ in each factor. We call the resulting extension $\mathcal{M}_{A}(\beta)$ of $M_{A}(\beta)$ the projectivized A-hypergeometric system defined by $A$ and $\beta$. We use the results of the previous sections to discuss, in this sequence, $\mathcal{M}_{A}(\beta)$ from the points of view of the previous sections: $(A, L)$ umbrella, $L$-characteristic variety, $L$-characteristic cycle, and, as a special case, its slopes along all projective coordinate subspaces. Once again the $(A, L)$-umbrella will play a pivotal role in our investigation of $\mathcal{M}_{A}(\beta)$.

Notation 5.1. We use the canonical embedding

$$
\mathbb{C}^{1}=\operatorname{Spec}\left(\mathbb{C}\left[x_{j}\right]\right) \longleftrightarrow \operatorname{Proj}\left(\mathbb{C}\left[y_{j}, y_{j}^{\prime}\right]\right)=\mathbb{P}_{\mathbb{C}}^{1}=\mathbb{C}^{1} \sqcup\{\infty\}
$$

induced by $x_{j}=y_{j} / y_{j}^{\prime}$ to embed $X=\operatorname{Spec}\left(\mathbb{C}\left[x_{1}, \ldots, x_{n}\right]\right)$ into $\left(\mathbb{P}_{\mathbb{C}}^{1}\right)^{n}=: \bar{X}$ as the complement of the variety of the ideal sheaf generated by $\prod_{j=1}^{n} y_{j}^{\prime}$. Recall from Notation 2.1 that the symbol $\bar{\tau}$ denotes the complement of a set $\tau$ in the set $\{1, \ldots, n\}$. On the other hand, $\bar{C}$ will always refer to the Zariski closure of a set $C$ in $T^{*} \bar{X}$. We denote by $x_{j}^{\prime}:=y_{j}^{\prime} / y_{j}$ the coordinate on $\mathbb{P}_{\mathbb{C}}^{1} \backslash\{0\}$. A subset $\mathfrak{P} \subseteq\{1, \ldots, n\}$ defines an affine patch $X_{\mathfrak{P}} \cong \mathbb{C}^{n}$ of $\bar{X}$ by

$$
X_{\mathfrak{P}}=\operatorname{Spec}\left(\mathbb{C}\left[\left\{x_{j} \mid j \notin \mathfrak{P}\right\} \cup\left\{x_{j}^{\prime} \mid j \in \mathfrak{P}\right\}\right]\right) \text {. }
$$

In particular, $X=X_{\emptyset}$ in this notation. If $\mathcal{F}$ is a sheaf on $\bar{X}$ then we denote its sections over $X_{\mathfrak{P}}$ by $F_{(\mathfrak{P})}:=\mathcal{F}\left(X_{\mathfrak{P}}\right)$. On the other hand, if $g$ is an $\bar{X}$-global section then we denote by $g_{(\mathfrak{P})}$ the restriction of $g$ to $X_{\mathfrak{P}}$.

Denote by $\mathcal{D}$ the sheaf of algebraic $\mathbb{C}$-linear differential operators on $\bar{X}$. Then with the above notation the ring $D_{(\mathfrak{P})}=\mathcal{D}\left(X_{\mathfrak{P}}\right)$ is the Weyl algebra in the variables $\left\{x_{j} \mid j \notin \mathfrak{P}\right\} \cup\left\{x_{j}^{\prime} \mid j \in \mathfrak{P}\right\}$. Let $\partial_{j}^{\prime}$ denote the derivation on $\mathbb{P}_{\mathbb{C}}^{1}$ relative to $x_{j}^{\prime}$. The sheaf $\mathcal{D}$ has a special subsheaf of rings $\mathcal{R}$, consisting entirely of the global differential operators in $R=\mathbb{C}[\partial]$. There are ring isomorphisms

$$
\rho_{\mathfrak{P}}: R_{(\emptyset)}=\mathbb{C}[\partial] \longrightarrow \mathbb{C}\left[\partial_{(\mathfrak{P})}\right]=\mathbb{C}\left[\partial_{\overline{\mathfrak{P}}}\right]\left[\left\{x_{j}^{\prime 2} \partial_{j}^{\prime} \mid j \in \mathfrak{P}\right\}\right]=R_{(\mathfrak{P})}
$$

defined by $\partial_{j} \mapsto-x_{j}^{\prime 2} \partial_{j}^{\prime}$ for $j \in \mathfrak{P}$. Any $\mathbb{Z}^{d}$-graded $R$-module induces a $\mathbb{Z}^{d}$-graded $\mathcal{R}$-module via equation (5.0.1).

Note that $x_{j}^{\prime}$ naturally inherits the $\mathbb{Z}^{d}$-degree $\operatorname{deg}\left(x_{j}^{\prime}\right):=-\operatorname{deg}\left(x_{j}\right)=\mathbf{a}_{j}$. Since $x_{j} \partial_{j}=-x_{j}^{\prime} \partial_{j}^{\prime}$, we put $\operatorname{deg}\left(\partial_{j}^{\prime}\right):=\operatorname{deg}\left(x_{j} \partial_{j}\right)-\operatorname{deg}\left(x_{j}^{\prime}\right)=-\mathbf{a}_{j}$. This makes $\mathcal{D}$ a $\mathbb{Z}^{d}$-graded sheaf of right $\mathcal{R}$-modules.

Similarly, let $L=\left(L_{x}, L_{\partial}\right)$ be a weight vector on $D=D_{\emptyset}$, so $L_{\partial}+L_{x} \geq 0$. We extend $L$ to the variables $x_{j}^{\prime}$ and $\partial_{j}^{\prime}$ by setting $L_{x_{j}^{\prime}}:=-L_{x_{j}}$ and $L_{\partial_{j}^{\prime}}:=L_{x_{j}}+L_{\partial_{j}}-$ $L_{x_{j}^{\prime}}$. This extends the $L$-filtration on $D=D_{(\emptyset)}$ to a global $L$-filtration on the sheaf $\mathcal{D}$. Let $\mathcal{W}:=\operatorname{gr}^{L}(\mathcal{D})$ be the sheaf of associated graded algebras.

By definition, $L_{\partial}+L_{x}=L_{\partial^{\prime}}+L_{x^{\prime}}$. Suppose $L_{x}+L_{\partial}>0$, then $W_{(\mathfrak{P})}=\operatorname{gr}^{L}\left(D_{(\mathfrak{P})}\right)$ is the polynomial ring $\mathbb{C}\left[x_{\mathfrak{P}}, \partial_{\mathfrak{P}}, x_{\overline{\mathfrak{P}}}^{\prime}, \partial_{\overline{\mathfrak{P}}}^{\prime}\right]$ and $\mathcal{W}$ can be interpreted as the sheaf of regular functions on $T^{*} \bar{X}$. 
Definitions 3.1 and 4.4 of the $L$-characteristic variety $\mathrm{Ch}^{L}$ and $L$-characteristic cycle $\mathrm{Ch}^{L}$ generalize to finite $\mathcal{D}$-modules $\mathcal{M}$ and homologically holonomic complexes $\mathcal{K}_{\bullet}$ of $\mathcal{D}$-modules. Both $L$-characteristic variety and cycle behave well under restriction to charts as $L$ is global.

Since both $\partial_{j}, x_{j} \partial_{j} \in D=D_{(\emptyset)}$ extend to global differential operators on $\bar{X}$, the same is true for the generators $\square_{\mathbf{u}}$ with $A \cdot \mathbf{u}=0$ and $E-\beta$ with $1 \leq i \leq n$ in Definition 3.5. We will denote these extended operators by the same symbols as the ones we used on $X_{\emptyset}$. This provides the motive for introducing a projectivized version of $M_{A}(\beta)$ as follows.

Definition 5.2. Define the $\mathcal{R}$-module $\mathcal{I}_{A}:=\mathcal{R} \cdot I_{A}$ as the extension of $I_{A}$ to $\bar{X}$. The projectivized A-hypergeometric system $\mathcal{M}_{A}(\beta)$ is the sheaf of $\mathcal{D}$-modules

$$
\mathcal{M}_{A}(\beta):=\frac{\mathcal{D}}{\mathcal{D}\left\langle I_{A}, E-\beta\right\rangle} .
$$

On the affine patch $X_{\mathfrak{P}}$, using notation introduced above, one has

$$
M_{A,(\mathfrak{P})}(\beta)=D_{(\mathfrak{P})} / D_{(\mathfrak{P})}\left\langle I_{A,(\mathfrak{P})}, E_{(\mathfrak{P})}-\beta\right\rangle .
$$

Note, how $I_{A,(\mathfrak{P})}=\rho_{\mathfrak{P}}\left(I_{A}\right)$ and $E_{(\mathfrak{P})}=\rho_{\mathfrak{P}}(E)$ behave under change of $\mathfrak{P}$ : moving the index $j$ into $\mathfrak{P}$ results in the change $a_{i, j} x_{j} \partial_{j} \mapsto-a_{i, j} x_{j}^{\prime} \partial_{j}^{\prime}$ in $E_{(\mathfrak{P})}$, and in the substitution $\partial_{j} \mapsto-x_{j}^{\prime 2} \partial_{j}^{\prime}$ in $I_{A,(\mathfrak{P})}$.

We begin our study of $\mathcal{M}_{A}(\beta)$ with a basic observation. Since the $L$-filtration is compatible with restriction to charts, we have $\left(\sigma^{L}(P)\right)_{(\mathfrak{P})}=\sigma^{L}\left(P_{(\mathfrak{P})}\right)$ for all global sections $P$ of $\mathcal{D}$. Thus the local sections of the ideal sheaf

$$
\mathcal{I}_{A}^{L}:=\operatorname{gr}^{L}\left(\mathcal{I}_{A}\right)=\mathcal{R}\left\langle\sigma^{L}\left(\square_{\mathbf{u}}\right) \mid A \cdot \mathbf{u}=0\right\rangle
$$

are given by $I_{A,(\mathfrak{P})}^{L}=\operatorname{gr}^{L}\left(I_{A,(\mathfrak{P})}\right)$. More interestingly, Buchberger's algorithm yields the following analog to Lemma 2.12 regarding the $L$-graded ideal of $\mathcal{D} \cdot I_{A}$.

Lemma 5.3. As ideal sheaves in $\mathcal{W}$,

$$
\operatorname{gr}^{L}\left(\mathcal{D} \cdot \mathcal{I}_{A}\right)=\mathcal{W} \cdot \mathcal{I}_{A}^{L}
$$

so that for all $\mathfrak{P}$ one has

$$
\operatorname{gr}^{L}\left(D_{(\mathfrak{P})} \cdot I_{A,(\mathfrak{P})}\right)=W_{(\mathfrak{P})} \cdot I_{A,(\mathfrak{P})}^{L}=W_{(\mathfrak{P})}\left\langle\sigma^{L}\left(\square_{\mathbf{u}}\right)_{(\mathfrak{P})} \mid \mathbf{u} \in \mathbb{Z}^{n}, A \cdot \mathbf{u}=0\right\rangle .
$$

We now study the variety of $T^{*} \bar{X}$ defined by $\mathcal{W} \cdot \mathcal{I}_{A}^{L}$. Since $\mathcal{I}_{A}^{L}$ is $\mathbb{Z}^{d}$-graded, the associated prime ideals of $\mathcal{W} \cdot \mathcal{I}_{A}^{L}$ will be $\mathbb{Z}^{d}$-graded as well and we hence search for $\mathbb{Z}^{d}$-graded sheaves of prime ideals $\mathcal{P} \subseteq \mathcal{W}$ containing $\mathcal{W} \cdot \mathcal{I}_{A}^{L}$. By Theorem 2.14 the $\mathbb{Z}^{d}$-graded prime ideals of $R_{(\emptyset)}$ containing $I_{A}^{L}$ are of the form $I_{A}^{\tau}$ with $\tau \in \Phi_{A}^{L}$. Since their sections are $\bar{X}$-global, every $\mathcal{P}$ as above must contain one of the ideals $\mathcal{I}_{A}^{\tau}:=\mathcal{R} \cdot I_{A}^{\tau}$ with $\tau \in \Phi_{A}^{L}$. Thus we shall look for the components of the variety

$$
\Upsilon_{A}^{\tau}:=\operatorname{Var}\left(\mathcal{W} \cdot \mathcal{I}_{A}^{\tau}\right) \subseteq T^{*} \bar{X}
$$

The sections $I_{A,(\mathfrak{P})}^{\tau}$ of $\mathcal{I}_{A}^{\tau}$ over a chart $X_{\mathfrak{P}}$ are the elements of $I_{A}^{\tau}$ with $\partial_{j}$ replaced by $-x_{j}^{\prime 2} \partial_{j}^{\prime}$ for all $j \in \mathfrak{P}$. On $T^{*} X_{\emptyset}, I_{A,(\emptyset)}^{\tau}=I_{A}^{\tau}$ defines an irreducible variety whose (again irreducible) closure in $T^{*} \bar{X}$ we denote by $\Upsilon_{A}^{\tau, \emptyset}$.

Assume first that $\mathfrak{P}$ is contained in $\tau$. In this case, $W_{(\mathfrak{P})} \cdot I_{A,(\mathfrak{P})}^{\tau}$ is generated by $I_{\tau,(\mathfrak{P})}$ and $\partial_{\overline{\tau \cup \mathfrak{P}}}$ which reduces the problem to understanding $W_{(\mathfrak{P})} \cdot I_{\tau,(\mathfrak{P})}$.

Lemma 5.4. If $\mathfrak{P} \subseteq \tau$ then the ideal $W_{(\mathfrak{P})} \cdot I_{\tau,(\mathfrak{P})}$ is a prime ideal. 
Proof. By equation (5.0.1) $I_{\tau,(\mathfrak{P})}=\rho_{\mathfrak{P}}\left(I_{\tau}\right)$ is prime in $R_{(\mathfrak{P})}$ and hence so is $I_{\tau,(\mathfrak{P})}$. $R_{(\mathfrak{P})}\left[x_{\mathfrak{P}}^{\prime}, x_{\overline{\mathfrak{P}}}\right]$. Now $W_{(\mathfrak{P})}=R_{(\mathfrak{P})}\left[x_{\mathfrak{P}}^{\prime}, x_{\mathfrak{P}}\right]\left[\partial_{\mathfrak{P}}^{\prime}\right]$ and $\partial_{j}^{\prime}=-\rho_{\mathfrak{P}}\left(\partial_{j}\right) / x_{j}^{\prime 2}$ for $j \in \mathfrak{P}$. Since for $j \in \mathfrak{P}, x_{j}^{\prime}$ is not in $R_{(\mathfrak{P})}\left[x_{\mathfrak{P}}^{\prime}, x_{\mathfrak{P}}\right] \cdot I_{\tau,(\mathfrak{P})}$ (and, by primeness, not in any associated prime either), it follows that under the ring extension $R_{\mathfrak{P}} \hookrightarrow W_{\mathfrak{P}}$ the ideal $I_{\tau,(\mathfrak{P})}$ remains prime.

It follows that for $\mathfrak{P} \subseteq \tau, \Upsilon_{A}^{\tau}$ is irreducible on $X_{\mathfrak{P}}$ and $W_{(\mathfrak{P})} \cdot I_{A,(\mathfrak{P})}^{\tau}$ is precisely the defining ideal of $\Upsilon_{A}^{\tau, \emptyset} \cap T^{*} X_{\mathfrak{P}}$.

We now view $\mathfrak{P}=(\mathfrak{P} \cap \tau) \sqcup(\mathfrak{P} \backslash \tau)$. By the above, $W_{(\mathfrak{P})} \cdot I_{A,(\mathfrak{P})}^{\tau}$ is the ideal of $W_{(\mathfrak{P})}$ generated by $I_{\tau,(\mathfrak{P})}, \partial_{\bar{\tau} \cup \mathfrak{P}}$ and $\left\{x_{j}^{\prime 2} \partial_{j}^{\prime} \mid j \in \mathfrak{P} \backslash \tau\right\}$. By Lemma 5.4 the radical of this ideal has a decomposition into prime ideals

$$
\sqrt{I_{A,(\mathfrak{P})}^{\tau}}=\bigcap_{\mathfrak{T} \subseteq \mathfrak{P} \backslash \tau} W_{(\mathfrak{P})}\left\langle I_{\tau,(\mathfrak{P})}, \partial_{\overline{\tau \cup \mathfrak{P}}}, x_{\mathfrak{T}}^{\prime}, \partial_{\mathfrak{P} \backslash(\mathfrak{T} \cup \tau)}^{\prime}\right\rangle .
$$

We are prompted to make the following definitions generalizing Definitions 2.2 and 2.7 .

Definition 5.5. Let

$$
\bar{\Phi}_{A}^{L}:=\left\{(\tau, \mathfrak{T}) \mid \tau \in \Phi_{A}^{L}, \mathfrak{T} \subseteq\{1, \ldots, n\}, \mathfrak{T} \cap \tau=\emptyset\right\}
$$

and $\bar{\Phi}_{A}^{L, k}:=\left\{(\tau, \mathfrak{T}) \in \bar{\Phi}_{A}^{L} \mid \tau \in \Phi_{A}^{L, k}\right\}$. For $(\tau, \mathfrak{T}) \in \bar{\Phi}_{A}^{L}$, put

$$
J_{\tau, \mathfrak{T},(\mathfrak{P})}:=R_{(\mathfrak{P})}\left\langle x_{\mathfrak{T}}^{\prime}, \partial_{\overline{\tau \cup \mathfrak{T} \cup \mathfrak{P}}}, \partial_{\overline{\tau \cup \mathfrak{T}} \cap \mathfrak{P}}^{\prime}\right\rangle
$$

and define the prime ideal sheaf $\mathcal{I}_{A}^{\tau, \mathfrak{T}} \supseteq \mathcal{W} \cdot I_{A}^{\tau} \supseteq \mathcal{W} \cdot I_{A}^{L}$ on each chart $X_{\mathfrak{P}}$ by

$$
I_{A,(\mathfrak{P})}^{\tau, \mathfrak{T}}:= \begin{cases}W_{(\mathfrak{P})}\left\langle I_{\tau,(\mathfrak{P})}, J_{\tau, \mathfrak{T},(\mathfrak{P})}\right\rangle, & \text { if } \mathfrak{P} \supseteq \mathfrak{T}, \\ W_{(\mathfrak{P})}, & \text { otherwise. }\end{cases}
$$

The irreducible varieties $\Upsilon_{A}^{\tau, \mathfrak{T}}:=\operatorname{Var}\left(\mathcal{I}_{A}^{\tau, \mathfrak{T}}\right) \subseteq T^{*} \bar{X}$ define quasi-affine varieties

$$
\dot{\Upsilon}_{A}^{\tau, \mathfrak{T}}:=\operatorname{Var}\left(I_{A,(\mathfrak{T})}^{\tau, \mathfrak{T}}\right) \backslash \bigcup_{\substack{\left(\tau^{\prime}, \mathfrak{T}\right) \in \bar{\Phi}_{A}^{L} \\ \tau^{\prime} \subseteq \tau}} \operatorname{Var}\left(I_{A,(\mathfrak{T})}^{\tau^{\prime}, \mathfrak{T}}\right) \subseteq T^{*} X_{\mathfrak{T}} .
$$

Note that $\Upsilon_{A}^{\tau, \mathfrak{T}}$ (and hence $\dot{\Upsilon}_{A}^{\tau, \mathfrak{T}}$ ) has dimension $\operatorname{dim}(\tau)+n$ and meets the chart $T^{*} X_{\mathfrak{P}}$ exactly if $\mathfrak{P} \supseteq \mathfrak{T}$. Let finally $\nu_{A}^{L, \tau, \mathfrak{T}}$ be the multiplicity of $\mathcal{I}_{A}^{L}$ along $\Upsilon_{A}^{\tau, \mathfrak{T}}$.

The quasi-affine varieties $\dot{\Upsilon}_{A}^{\tau, \mathfrak{T}}$ will play the role of the orbits in Section 2, In fact, the action of the $d$-torus $\mathbb{T}$ on $T_{0}^{*} X$ can be extended to $\bar{X}$ via the extension of the coordinate ring $R$ of $T_{0}^{*} X$ : Denote

$$
Z_{\mathfrak{P}}:=\operatorname{Var}\left(x_{\mathfrak{P}}^{\prime}\right) \subseteq X_{\mathfrak{P}} .
$$

For $p \in Z_{\mathfrak{P}},\left\{\partial_{j} \mid j \notin \mathfrak{P}\right\} \cup\left\{\partial_{j}^{\prime} \mid j \in \mathfrak{P}\right\}$ are coordinates on $T_{p}^{*} \bar{X}$. Similar to (2.1.1), $t \in \mathbb{T}$ applies componentwise to $\xi=\left(\xi, \xi^{\prime}\right) \in T_{p}^{*} \bar{X}$ by $t \cdot \xi_{j}=t^{\mathbf{a}_{j}} \xi_{j}$ but $t \cdot \xi_{j}^{\prime}=0$ because $\partial_{j}=-x_{j}^{\prime 2} \partial_{j}^{\prime}$ vanishes on $Z_{\mathfrak{P}}$. When restricted to $T_{p}^{*} Z_{\mathfrak{P}}$, this becomes a group action uniform in $p \in Z_{\mathfrak{P}}$. To generalize Definition 2.2 , let $(\tau, \mathfrak{T}) \in \bar{\Phi}_{A}^{L}$, denote by $\mathbf{1}_{A}^{\tau, \mathfrak{T}}$ the point $\mathbf{1}_{A}^{\tau}$ in $T_{0}^{*} Z_{\mathfrak{T}}$, and set

$$
O_{A}^{\tau, \mathfrak{T}}:=\operatorname{Orb}\left(\mathbf{1}_{A}^{\tau, \mathfrak{T}}\right) \subseteq T_{0}^{*} Z_{\mathfrak{T}}
$$


By Lemma 2.3. $O_{A}^{\tau, \mathfrak{T}}=\operatorname{Var}\left(I_{A \backslash \mathfrak{T}}^{\tau}\right)$ so that $O_{A}^{\tau, \mathfrak{T}} \times \mathbb{C}^{|\mathfrak{T}|}$ identifies with $O_{A}^{\tau}$. The inclusion $Z_{\mathfrak{T}}=\operatorname{Var}\left(x_{\mathfrak{T}}^{\prime}\right) \subseteq X_{\mathfrak{T}}$ induces natural maps of cotangent spaces

$$
T^{*} X_{\mathfrak{T}} \stackrel{\iota_{\mathfrak{T}}}{\longrightarrow} T^{*} X_{\mathfrak{T} \mid Z_{\mathfrak{T}}} \stackrel{\pi_{\mathfrak{T}}}{\longrightarrow} T^{*} Z_{\mathfrak{T}} .
$$

and $\dot{\Upsilon}_{A}^{\tau, \mathfrak{T}}=\iota_{\mathfrak{T}}\left(\pi_{\mathfrak{T}}^{-1}\left(O_{A}^{\tau, \mathfrak{T}} \times Z_{\mathfrak{T}}\right)\right)$. The partial Fourier transform

$$
W_{(\mathfrak{T})} \longrightarrow W_{(\emptyset)}, \quad x_{\mathfrak{T}}^{\prime} \longmapsto \partial_{\mathfrak{T}}, \quad \partial_{\mathfrak{T}}^{\prime} \longmapsto-x_{\mathfrak{T}},
$$

identifies $\dot{\Upsilon}_{A}^{\tau, \mathfrak{T}}$ with the product $O_{A}^{\tau} \times \mathbb{C}^{n}$

By the preceding discussion, Theorem 2.14 and Proposition 2.17 can be generalized as follows.

Theorem 5.6. A decomposition of $\Upsilon_{A}^{L}$ into irreducible components is given by

$$
\Upsilon_{A}^{L}=\bigcup_{(\tau, \mathfrak{T}) \in \bar{\Phi}_{A}^{L, d-1}} \Upsilon_{A}^{\tau, \mathfrak{T}}
$$

More generally, there is a stratification

$$
\Upsilon_{A}^{L}=\bigsqcup_{(\tau, \mathfrak{T}) \in \bar{\Phi}_{A}^{L}} \dot{\Upsilon}_{A}^{\tau, \mathfrak{T}}
$$

such that $\dot{\Upsilon}_{A}^{\tau^{\prime} \mathfrak{T}^{\prime}}$ is in the closure of $\dot{\Upsilon}_{A}^{\tau, \mathfrak{T}}$ if and only if $\tau^{\prime} \subseteq \tau$ and $\tau \backslash \tau^{\prime} \subseteq \mathfrak{T}^{\prime} \supseteq \mathfrak{T}$. For $(\tau, \mathfrak{T}) \in \bar{\Phi}_{A}^{L, d-1}$, the multiplicity of $\Upsilon_{A}^{\tau, \mathfrak{T}}$ in $\Upsilon_{A}^{L}$ is

$$
\nu_{A}^{L, \tau, \mathfrak{T}}=2^{|\mathfrak{T}|} \cdot \nu_{A}^{L, \tau}=2^{|\mathfrak{T}|} \cdot\left[\mathbb{Z}^{d}: \mathbb{Z} \tau\right] .
$$

Proof. The irreducible decomposition is a consequence of Lemma 5.4, The existence of the stratification follows from Theorem 2.14 and the preceding discussion. For $j \in \tau \backslash \mathfrak{P}, \rho_{\mathfrak{P} \cup\{j\}}\left(\partial_{j}\right)=-{x_{j}^{\prime}}^{2} \partial_{j}^{\prime}$ and the adjacencies in Theorem 2.14 imply that

$$
\sqrt{\left\langle I_{A,(\mathfrak{T} \cup\{j\})}^{\tau, \mathfrak{T}}, x_{j}^{\prime}\right\rangle}=\bigcap_{\tau^{\prime} \subseteq \tau \backslash\{j\}} I_{A,(\mathfrak{T} \cup\{j\})}^{\tau^{\prime}, \mathfrak{T} \cup\{j\}}
$$

which leads to the adjacencies of the $\dot{\Upsilon}_{A}^{\tau, \mathfrak{T}}$. The multiplicity $\nu_{A}^{A, \tau, \mathfrak{T}}$ can be measured on the chart $X_{\mathfrak{T}}$. Then the statement is a consequence of the fact that the ideal generated by $x_{j}^{\prime 2} \partial_{j}^{\prime}$ has multiplicity two along $x_{j}^{\prime}=0$, combined with the formula for $\nu_{A}^{L, \tau}$ in Proposition 2.17 .

This ends our discussion of the globalization of the toric ideal $I_{A}$. From now on we assume that $L_{x_{j}}+L_{\partial_{j}}=c>0$ for all $j$ and investigate the projectivized $A$-hypergeometric system $\mathcal{M}_{A}(\beta)$. In that case, $E_{(\mathfrak{P})}$ is $L$-homogeneous of $L$-degree $c$ for every chart index $\mathfrak{P}$. We imitate Definition 3.7 as follows.

Definition 5.7. For $(\tau, \mathfrak{T}) \in \bar{\Phi}_{A}^{\tau, \mathfrak{T}}$, set $C_{A}^{\tau, \mathfrak{T}}:=\dot{\Upsilon}_{A}^{\tau, \mathfrak{T}} \cap \operatorname{Var}\left(E_{(\mathfrak{T})}\right) \subseteq T^{*} X_{\mathfrak{T}}$ and let $\mathcal{P}_{\tau, \mathfrak{T}} \subseteq \mathcal{W}$ be the prime ideal sheaf of $\bar{C}_{A}^{\tau, \mathfrak{T}}$.

The isomorphism of $\dot{\Upsilon}_{A}^{\tau, \mathfrak{T}}$ with $O_{A}^{\tau} \times \mathbb{C}^{n}$ via the partial Fourier transform (5.0.2) mentioned above identifies $E_{(\mathfrak{T})}$ with $E_{(\emptyset)}$. Thus $C_{A}^{\tau, \mathfrak{T}}$ is a quasi-affine variety isomorphic to $C_{A}^{\tau}$. This yields the following estimate for the $L$-characteristic variety of $\mathcal{M}_{A}(\beta)$ in the spirit of Proposition 3.8 
Proposition 5.8. The characteristic variety of $\mathcal{M}_{A}(\beta)$ is contained in the union of the closures of the Fourier twisted orbit conormals:

$$
\operatorname{Ch}\left(\mathcal{M}_{A}(\beta)\right) \subseteq \bigcup_{(\tau, \mathfrak{T}) \in \Phi_{A}^{L}} \bar{C}_{A}^{\tau, \mathfrak{T}}
$$

We now wish to compute the actual components and their multiplicities in the characteristic cycle of $\mathcal{M}_{A}(\beta)$. We proceed exactly as in Section 4 Multiplicities along a candidate component $\bar{C}_{A}^{\tau, \mathfrak{T}}$ where $(\tau, \mathfrak{T}) \in \bar{\Phi}_{A}^{L}$ can be computed on the chart $X_{\mathfrak{T}}$. This means the $\mathcal{D}$-module $\mathcal{M}_{A}(\beta)$ can be replaced by the $D_{(\mathfrak{T})}$-module $M_{A,(\mathfrak{P})}(\beta)$. Parallel to the affine case in Definition 4.6, one can introduce a global Euler-Koszul complex on $\bar{X}$.

Definition 5.9. Let $N$ be a $\mathbb{Z}^{d}$-graded $R$-module and $\beta \in \mathbb{C}^{d}$. We consider $\mathcal{D}$ as a $\mathbb{Z}^{d}$-graded right $\mathcal{R}$-module and $N$ as a $\mathbb{Z}^{d}$-graded $\mathcal{R}$-module as in Notation 5.1 Then $\mathcal{D} \otimes_{\mathcal{R}} N$ is a $\mathbb{Z}^{d}$-graded left $\mathcal{D}$-module. On a $\mathbb{Z}^{d}$-graded left $\mathcal{D}$-module $\mathcal{M}$, the assignment (4.2.1) and $\mathcal{D}$-linear extension yield $d$ commuting $\mathcal{D}$-linear endomorphisms $E-\beta$. We define the global Euler-Koszul complex $\mathcal{K}_{A, \bullet}(N ; E-\beta)$ and the global Euler-Koszul homology $\mathcal{H}_{A, \bullet}(N ; E-\beta)$ as the sheafified version of the objects in Definition 4.6. Note that $\mathcal{H}_{A, 0}\left(S_{A} ; E-\beta\right)=\mathcal{M}_{A}(\beta)$.

A good behavior of this global Euler-Koszul functor such as a long exact sequence of Euler-Koszul homology requires flatness of $\mathcal{D}$ over $\mathcal{R}$.

Lemma 5.10. The sheaves of $\mathcal{R}$-modules $\mathcal{D}$ and $\mathcal{W}$ are locally free and hence flat.

Proof. It suffices to consider the single variable case on a chart $X_{\mathfrak{P}}$. For $j \in \mathfrak{P}$, $\mathbb{C}\left[x_{j}^{\prime}, \partial_{j}^{\prime}\right]$ is a free $\mathbb{C}\left[-x_{j}^{\prime 2} \partial_{j}^{\prime}\right]$-module with basis $x_{j}^{\prime k} \partial_{j}^{\prime l}$ where $k \leq 1$ or $l=0$. Since $\mathbb{C}\left[x_{j}^{\prime}, \partial_{j}^{\prime}\right]=\operatorname{gr}^{F}\left(\mathbb{C}\left[x_{j}^{\prime}\right]\left\langle\partial_{j}^{\prime}\right\rangle\right)$ the same is true for $\mathbb{C}\left[x_{j}^{\prime}\right]\left\langle\partial_{j}^{\prime}\right\rangle$.

The Euler-Koszul homology sheaves of a toric $R$-module are holonomic for reasons similar to those given in MMW05 for Euler-Koszul homology modules. Their $L$-characteristic variety is hence $n$-dimensional by Proposition 3.8 and Smi01. A spectral sequence argument like in Theorem 4.11 shows the possible components of $\mathrm{Ch}^{L}\left(\mathcal{H}_{A, \bullet}(N ; \beta)\right)$ are of the form $\bar{C}_{A}^{\tau, \mathfrak{T}}$ with $(\tau, \mathfrak{T}) \in \bar{\Phi}_{A}^{L}$.

Definition 5.11. For $(\tau, \mathfrak{T}) \in \bar{\Phi}_{A}^{L}$ and $i \in \mathbb{N}$, we denote by $\mu_{A, i}^{L, \tau, \mathfrak{T}}(\beta)$ the multiplicity of $\operatorname{gr}^{L}\left(\mathcal{H}_{A, i}\left(S_{A} ; \beta\right)\right)$ along $\bar{C}_{A}^{\tau, \mathfrak{T}}$ and put $\mu_{A}^{L, \tau, \mathfrak{T}}:=\sum_{i=1}^{d}(-1)^{i} \mu_{A, i}^{L, \tau, \mathfrak{T}}(\beta)$.

By looking at the chart $X_{\mathfrak{T}}$ it follows from the appendix that, as in the affine case, the alternating sum of the $\mu_{A, i}^{L, \tau, \mathfrak{T}}(\beta)$ agrees with the intersection multiplicity of $I_{A,(\mathfrak{T})}^{L}$ with $E_{(\mathfrak{T})}$ in the local ring at $\bar{C}_{A}^{\tau, \mathfrak{T}}$. By Theorem 5.6, this reduces to the intersection multiplicity of $I_{A,(\mathfrak{T})}^{\tau, \mathfrak{T}}$ with $E_{(\mathfrak{T})}$, multiplied by $\nu_{A}^{L, \tau, \mathfrak{T}}=2^{|\mathfrak{T}|} \nu_{A}^{L, \tau}$. From Theorem 4.11 we conclude the following statement which shows in particular that $\mu_{A}^{L, \tau, \mathfrak{T}}$ is independent of $\beta$ as suggested by the notation. For the sake of simplicity we concentrate on the case of interest $N=S_{A}$.

Theorem 5.12. For all $(\tau, \mathfrak{T}) \in \bar{\Phi}_{A}^{L}$ and all $\beta \in \mathbb{C}^{d}$,

$$
\mu_{A}^{L, \tau, \mathfrak{T}}=\chi^{C}\left(\mathcal{W} / \mathcal{W}\langle E\rangle, \mathcal{W} \otimes_{\mathcal{R}} S_{A}^{L}\right)=2^{|\mathfrak{T}|} \mu_{A}^{L, \tau},
$$

where $C=\bar{C}_{A}^{\tau, \mathfrak{T}}$, is independent of $\beta$ and positive. 
In [MMW05, Thm. 6.3], a spectral sequence is constructed that is used to show good vanishing properties of Euler-Koszul homology. We leave it to the reader to verify that it generalizes to our global context. It follows that if $\beta$ is generic then $\mathcal{K}_{A, \bullet}\left(S_{A}, E-\beta\right)$ has only homology in degree zero. In particular, $\mu_{A, 0}^{L, \tau, \mathfrak{T}}(\beta)=$ $\mu_{A}^{L, \tau, \mathfrak{T}}>0$ for generic $\beta$, similar to Corollary 4.12 .

In order to show nonvanishing for all $\beta$ one can generalize Theorems 3.10 and 4.28 by following exactly the steps of our proof in the chart $X_{\mathfrak{T}}$ corresponding to $\bar{C}_{A}^{\tau, \mathfrak{T}}$, always twisting the toric input module by $\rho_{\mathfrak{P}}$ from equation (5.0.1).

Theorem 5.13. The L-characteristic variety of $\mathcal{M}_{A}(\beta)$ is given by

$$
\operatorname{Ch}^{L}\left(\mathcal{M}_{A}(\beta)\right)=\bigcup_{(\tau, \mathfrak{T}) \in \bar{\Phi}_{A}^{L}} \bar{C}_{A}^{\tau, \mathfrak{T}}
$$

For all $(\tau, \mathfrak{T}) \in \bar{\Phi}_{A}^{L}$ and all $\beta \in \mathbb{C}^{d}$,

$$
\mu_{A, 0}^{L, \tau, \mathfrak{T}}(\beta) \geq \mu_{A}^{L, \tau, \mathfrak{T}}=2^{|\mathfrak{T}|} \mu_{A}^{L, \tau}
$$

is positive, and equality holds for generic $\beta$. In particular, the L-characteristic variety of $\mathcal{M}_{A}(\beta)$ is entirely determined by the $(A, L)$-umbrella $\Phi_{A}^{L}$. For $\tau \in \Phi_{A}^{L, n-1}$,

$$
\mu_{A}^{L, \tau, \mathfrak{T}}=2^{|\mathfrak{T}|} \nu_{A}^{L, \tau}=2^{|\mathfrak{T}|}\left[\mathbb{Z}^{d}: \mathbb{Z} \tau\right]
$$

In order to study slopes of $\mathcal{M}_{A}(\beta)$ along coordinate varieties, we consider $\mathfrak{V}$ as a partitioned set $\mathfrak{V}=\mathfrak{V}_{0} \sqcup \mathfrak{V}_{\infty} \subseteq\{1, \ldots, n\}$. As in (3.1.1), $\mathfrak{V}$ defines the coordinate variety

$$
Y:=\operatorname{Var}\left(x_{\mathfrak{V}_{0}}, x_{\mathfrak{V}_{\infty}}^{\prime}\right) \subseteq \bar{X}
$$

which meets the chart $X_{\mathfrak{P}}$ exactly if $\mathfrak{V}_{0} \cap \mathfrak{P}=\emptyset$ and $\mathfrak{V}_{\infty} \subseteq \mathfrak{P}$. On any such $X_{\mathfrak{P}}$, $Y$ induces the family of filtrations

$$
L=p F+q V, \quad p / q \in \mathbb{Q}_{>0} \cup\{\infty\},
$$

on $D_{\mathfrak{P}}$ as in (3.1.2) where $V$ is the $V$-filtration along $Y \cap X_{\mathfrak{P}}$ defined by the assignment $-V_{x_{i}}=1=V_{\partial_{i}}$ for $i \in \mathfrak{V}_{0}, V_{x_{i}^{\prime}}=1=-V_{\partial_{i}^{\prime}}$ for $i \in \mathfrak{V}_{\infty}$, and $V_{x_{i}^{\prime}}=0=-V_{\partial_{i}^{\prime}}$ for $i \notin \mathfrak{V}$. One may view the patch $W_{\mathfrak{P}}=\operatorname{gr}^{L}\left(D_{\mathfrak{P}}\right)$ of $\mathcal{W}$ as the ring of polynomial functions on $T^{*}\left(T_{Y}^{*} X_{\mathfrak{P}}\right)$ and identify $T^{*} T_{Y}^{*} \bar{X}=T^{*}\left(\bar{X} \backslash \operatorname{Var}\left(x_{\mathfrak{V}_{0}}^{\prime}, x_{\mathfrak{V}_{\infty}}\right)\right)$. By Definition 3.4. $L=p / q$ a slope of a finite $\mathcal{D}$-module $\mathcal{M}$ on $\bar{X}$ at $y \in Y$ along $Y$ if the set of components of $\mathrm{Ch}^{L}(\mathcal{M})$ which meet $T_{y}^{*} \bar{X}$ jumps at $L$.

Recall that all slopes of $M_{A}(\beta)$ along coordinate varieties are slopes at the origin in $X$ by Lemma 3.14. But the following example shows that jumps of $\mathrm{Ch}^{L}\left(\mathcal{M}_{A}(\beta)\right)$ can be irrelevant for the slopes along $Y$.

Example 5.14. Let $A=\left(\begin{array}{lll}3 & 1 & 0 \\ 0 & 1 & 3\end{array}\right)$, pick $Y=\operatorname{Var}\left(x_{1}^{\prime}, x_{2}^{\prime}\right)$ and $L=p F+q V$ with $p>0, q \geq 0$ in $\mathbb{Q}$. Since $I_{A}^{L}$ is Cohen-Macaulay, $\operatorname{gr}^{L}\left(\mathcal{W}\left(I_{A}, E-\beta\right)\right)$ equals $\left\langle\partial_{2}^{3}, 3 x_{1} \partial_{1}+x_{2} \partial_{2}, x_{2} \partial_{2}+3 x_{3} \partial_{3}\right\rangle$ if $p / q>2$ but $\left\langle\partial_{1} \partial_{3}, 3 x_{1} \partial_{1}+x_{2} \partial_{2}, x_{2} \partial_{2}+3 x_{3} \partial_{3}\right\rangle$ if $p / q<2$. It follows that $\operatorname{Ch}^{L}\left(\mathcal{M}_{A}(\beta)\right)$ is defined by $\left\langle\partial_{2}, x_{1} \partial_{1}, x_{3} \partial_{3}\right\rangle$ for $p / q>$ 2 and by $\left\langle x_{2} \partial_{2}, \partial_{1} \partial_{3}, x_{3} \partial_{3}, x_{1} \partial_{1}\right\rangle$ if $p / q<2$. Thus, 2 is the only possible slope value and, as $p / q$ passes through 2 from above, the component of $\mathrm{Ch}^{L}\left(\mathcal{M}_{A}(\beta)\right)$ to $\left\langle x_{1}, \partial_{2}, x_{3}\right\rangle$ is replaced by the components to $\left\langle x_{2}, \partial_{1} \partial_{3}, x_{3} \partial_{3}, x_{1} \partial_{1}\right\rangle$. However, as $Y$ is defined by $x_{1}=x_{2}=\infty$, none of these components meet $Y$ and hence $\mathcal{M}_{A}(\beta)$ is regular along $Y$. 
It follows that Theorem 5.13 does not give directly a combinatorial description of the slopes of $\mathcal{M}_{A}(\beta)$ along $Y$. We must select the components of $\mathrm{Ch}^{L}\left(\mathcal{M}_{A}(\beta)\right)$ whose projection meets $Y$. For this, we look at a chart $X_{\mathfrak{P}}$ which meets $Y$ and hence $\mathfrak{P} \supseteq \mathfrak{V}_{\infty}$ and $\mathfrak{P} \cap \mathfrak{V}_{0}=\emptyset$. Consider the component $\bar{C}_{A}^{\tau, \mathfrak{T}}$ of $\operatorname{Ch}^{L}\left(\mathcal{M}_{A}(\beta)\right)$ for some $(\tau, \mathfrak{T}) \in \bar{\Phi}_{A}^{L}$. Since $I_{A,(\mathfrak{P})}^{\tau, \mathfrak{T}}$ contains $x_{\mathfrak{T}}^{\prime}, \bar{C}_{A}^{\tau, \mathfrak{T}}$ can be visible in $X_{\mathfrak{P}}$ only if $\mathfrak{P} \supseteq \mathfrak{T}$. On the other hand, if $\bar{C}_{A}^{\tau, \mathfrak{T}}$ is not visible in $X_{\mathfrak{P}}$ then (since $\bar{C}_{A}^{\tau, \mathfrak{T}}$ is irreducible) its defining ideal contains an $x_{i}$ with $i \in \mathfrak{P}$. This may be the case even if $\mathfrak{P} \supseteq \mathfrak{T}$.

Definition 5.15. $\tau \in \Phi_{A}^{L}$ is a pyramid with vertex $i \in \tau$ if $\operatorname{dim}(\tau \backslash\{i\})<\operatorname{dim}(\tau)$.

Lemma 5.16. Let $(\tau, \mathfrak{T}) \in \bar{\Phi}_{A}^{L}$. There is no chart $X_{\mathfrak{P}}$ that meets both $Y$ and $\bar{C}_{A}^{\tau, \mathfrak{T}}$ if and only if either $\mathfrak{T} \cap \mathfrak{V}_{0} \neq \emptyset$ or $\tau$ is a pyramid with vertex in $\mathfrak{V}_{\infty}$. Moreover, if $\bar{C}_{A}^{\tau, \mathfrak{T}}$ is a component of $\mathrm{CC}^{L^{\prime}}\left(\mathcal{M}_{A}(\beta)\right)$ for $L^{\prime}$ near $L$ and meets some chart $X_{\mathfrak{P}}$ which meets $Y$ then its projection also meets $Y$.

Proof. To prove the first statement, assume that $\mathfrak{T} \cap \mathfrak{V}_{0}=\emptyset$ and $\bar{C}_{A}^{\tau, \mathfrak{T}}$ does not meet the chart $X_{\mathfrak{P}}$ with $\mathfrak{P}=\mathfrak{V}_{\infty} \cup \mathfrak{T}$. Then $C_{A}^{\tau, \mathfrak{T}} \subseteq \operatorname{Var}\left(x_{i}\right)$ for some $i \in \mathfrak{V}_{\infty}$ by the preceding discussion. Since $C_{A}^{\tau, \mathfrak{T}}$ is the conormal to $O_{A}^{\tau, \mathfrak{T}}$, the ideal $I_{A}^{\tau, \mathfrak{T}}$ is hence independent of of $\partial_{i}$. By definition of $I_{A}^{\tau, \mathfrak{T}}$ through the toric ideal $I_{\tau}$, this is equivalent to $\tau$ being a pyramid with vertex $i$. The converse implication of the first statement is obvious.

Under the hypothesis of the second statement, the ideal $\mathcal{P}_{\tau, \mathfrak{T}}$ of $\bar{C}_{A}^{\tau, \mathfrak{T}}$ is an associated prime of an $L$ - and an $L^{\prime}$-homogeneous ideal for some $L^{\prime}$ near $L$. As such, $\mathcal{P}_{\tau, \mathfrak{T}}$ is $\left(L, L^{\prime}\right)$ - and hence $(F, V)$-bihomogeneous. It follows that the nontrivial $W_{(\mathfrak{P})}$-ideal $P^{\prime}=P_{\tau, \mathfrak{T},(\mathfrak{P})}+\left\langle\partial_{\overline{\mathfrak{P}}}, \partial_{\mathfrak{P}}^{\prime}\right\rangle$ is $(F, V)$-bihomogeneous, generated by $\left\langle\partial_{\overline{\mathfrak{P}}}, \partial_{\mathfrak{P}}^{\prime}\right\rangle$ and some $\partial$-independent $V$-homogeneous elements. In particular, $P^{\prime}+\left\langle x_{\mathfrak{V}_{0}}, x_{\mathfrak{V}_{\infty}}^{\prime}\right\rangle$ is nontrivial and so $\bar{C}_{A}^{\tau, \mathfrak{T}}$ meets $Y$.

From the dimension one case, it is clear that non- $(F, V)$-bihomogeneous components are relevant for slopes. Indeed, the $L$-characteristic variety equals $\operatorname{Var}\left(x_{1} \partial_{1}\right)$ for most $L$-homogeneous differential operators in one variable $x_{1}$. However we believe that the non- $(F, V)$-bihomogeneous components are irrelevant in our case. More precisely, it seems that the conormal space of an $L$-homogeneous non- $(F, V)$ bihomogeneous pointed toric ring can not meet any point $T_{y}^{*} \bar{X}$ with $y \in Y$ unless $\mathfrak{V}_{\infty}=\emptyset$. This would imply the following combinatorial description of slopes of projectivized $A$-hypergeometric systems along coordinate varieties.

Definition 5.17. Let $L=p F+q V$ with $p / q \in \mathbb{Q}_{>0} \cup\{\infty\}$ where $V$ is the $V$ filtration along $Y=\operatorname{Var}\left(x_{\mathfrak{V}_{0}}, x_{\mathfrak{V}_{\infty}}^{\prime}\right)$. By $\Phi_{A}^{Y}(p / q)$ we denote the subset of $\tau \in \Phi_{A}^{L}$ which are not a pyramid with vertex in $\mathfrak{V}_{\infty}$.

Conjecture 5.18. The slopes of the projectivized A-hypergeometric system $\mathcal{M}_{A}(\beta)$ along a coordinate variety $Y$ are the jump parameters $p / q \in \mathbb{Q}_{>0} \cup\{\infty\}$ of $\Phi_{A}^{Y}(p / q)$.

\section{Appendix A. On spectral SEQuences}

Let $\left(K^{\bullet}, d\right)$ be a complex of groups equipped with a descending filtration $F$ of subgroups such that $d\left(F^{p} K^{\bullet}\right) \subseteq F^{p} K^{\bullet+1}$. We stress that there are no boundedness conditions on the filtration. We abbreviate $\mathrm{gr}=\mathrm{gr}_{F}$ and freely use induced filtrations on subgroups and quotient groups. 
By God58, Ch. I, Thm. 4.2.1, Thm. 4.2.2] there is an associated spectral sequence involving the following data:

$$
\begin{aligned}
& Z_{r}^{p, q}=F^{p} K^{p+q} \cap d^{-1}\left(F^{p+r} K^{p+q+1}\right), \\
& B_{r}^{p, q}=F^{p} K^{p+q} \cap d\left(F^{p-r} K^{p+q-1}\right), \\
& E_{r}^{p, q}=\operatorname{gr}^{p} \frac{d^{-1}\left(F^{p+r} K^{p+q+1}\right)}{d\left(F^{p-r+1} K^{p+q-1}\right)}=\operatorname{gr}^{p} \frac{Z_{r}^{p, q}}{B_{r-1}^{p, q}}=Z_{r}^{p, q} /\left(Z_{r-1}^{p+1, q-1}+B_{r-1}^{p, q}\right) .
\end{aligned}
$$

Note that $Z_{r}^{p, q} \cap F^{p+1} K^{p+q}=Z_{r-1}^{p+1, q-1}, d\left(Z_{r}^{p-r, q+r-1}\right)=B_{r}^{p, q}, E_{0}^{p, q}=\operatorname{gr}^{p} K^{p+q}$, and $E_{1}^{p, q}=H^{p+q}\left(\operatorname{gr}^{p}\left(K^{\bullet}\right), \operatorname{gr}^{p}(d)\right)$.

For all $p, q$ and $r, d$ induces a group homomorphism $d_{r}^{p, q}: E_{r}^{p, q} \rightarrow E_{r}^{p+r, q-r+1}$ such that $d_{r}^{p, q} \circ d_{r}^{p-r, q+r+1}=0$. More explicitly,

$$
d_{r}^{p, q}\left(z \bmod \left(Z_{r-1}^{p+1, q-1}+B_{r-1}^{p, q}\right)\right)=d(z) \bmod \left(Z_{r-1}^{p+1+r, q-r}+B_{r-1}^{p+r, q-r+1}\right)
$$

and $E_{r}^{p, q}=\left(\operatorname{ker} d_{r}^{p, q}\right) /\left(\operatorname{im} d_{r}^{p-r, q+r-1}\right)$. Let

$$
\begin{aligned}
& Z_{\infty}^{p, q}=F^{p} K^{p+q} \cap d^{-1}(0), \\
& B_{\infty}^{p, q}=F^{p} K^{p+1} \cap d\left(K^{p+q-1}\right) \\
& E_{\infty}^{p, q}=\operatorname{gr}^{p} H^{p+q}\left(K^{\bullet}\right)=\operatorname{gr}^{p} \frac{Z_{\infty}^{p, q}}{B_{\infty}^{p, q}}=Z_{\infty}^{p, q} /\left(Z_{\infty}^{p+1, q-1}+B_{\infty}^{p, q}\right) .
\end{aligned}
$$

In general, there is no relation between the terms $E_{r}^{p, q}$ for finite $r$ and $E_{\infty}^{p, q}$. The purpose of this section is to prove the following theorem and its corollary below.

Theorem A.1. Let $D$ be a ring and $\left(K^{\bullet}, d\right)$ be a complex of D-modules, both equipped with a descending filtration $F$ of subgroups subject to the hypotheses:

(a) $D$ is a filtered ring: $\left(F^{p} D\right) \cdot\left(F^{p^{\prime}} D\right) \subseteq F^{p+p^{\prime}} D$.

(b) $K^{\bullet}$ is a filtered D-module: $\left(F^{p} D\right) \cdot\left(F^{p^{\prime}} K^{n}\right) \subseteq F^{p+p^{\prime}} K^{n}$.

(c) $d$ is compatible with $F: d\left(F^{p} K^{p+q}\right) \subseteq F^{p} K^{p+q+1}$.

(d) $E_{0}=\operatorname{gr}\left(K^{\bullet}\right)$ is a Noetherian $\operatorname{gr}(D)$-module.

(e) $F$ is exhaustive and separated on $K^{\bullet}: \bigcup_{p} F^{p} K^{n}=K^{n}, \bigcap_{p} F^{p} K^{n}=0$.

Then one has the consequences:

(1) $d_{r}: E_{r} \rightarrow E_{r}$ are graded $\operatorname{gr}(D)$-linear maps of graded $\operatorname{gr}(D)$-modules with $\operatorname{gr}^{p^{\prime}}(D) \cdot E_{r}^{p, q} \subseteq E_{r}^{p+p^{\prime}, q-p^{\prime}}$

(2) $E_{r}$ is Noetherian and $d_{r}$ is the zero map for all sufficiently large $r$.

(3) The stable value of $E_{r}$ is isomorphic to $E_{\infty}$.

Proof. We abbreviate $W=\operatorname{gr}(D)$.

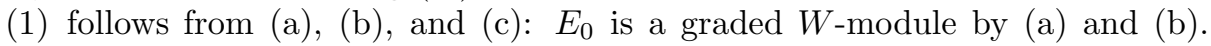
By induction, we may assume that $E_{r}$ is a graded $W$-module and $d_{r-1}$ is graded $W$-linear. Let $\delta \in F^{p^{\prime}} D$ and $c \in Z_{r}^{p, q}$ represent non zero elements $\bar{\delta} \in W^{p^{\prime}}$ and 
$\bar{c} \in E_{r}^{p, q}$. By (A.2) and (ㄷ),

$$
\begin{aligned}
d_{r}(\bar{\delta} \cdot \bar{c}) & =d_{r}\left(\left(\delta \bmod F^{p^{\prime}+1} D\right) \cdot\left(c \bmod \left(Z_{r-1}^{p+1, q-1}+B_{r-1}^{p, q}\right)\right)\right) \\
& =d_{r}\left(\delta c \bmod \left(Z_{r-1}^{p+p^{\prime}+1, q-p^{\prime}-1}+B_{r-1}^{p+p^{\prime}, q-p^{\prime}}\right)\right) \\
& =d(\delta c) \bmod \left(Z_{r-1}^{p+p^{\prime}+r+1, q-p^{\prime}-r-1}+B_{r-1}^{p+p^{\prime}+r, q-r-p^{\prime}}\right) \\
& =\delta d(c) \bmod \left(Z_{r-1}^{p+p^{\prime}+r+1, q-p^{\prime}-r-1}+B_{r-1}^{p+p^{\prime}+r, q-r-p^{\prime}}\right) \\
& =\left(\delta \bmod F^{p^{\prime}+1} D\right) \cdot\left(d(c) \bmod \left(Z_{r-1}^{p+r+1, q-r-1}+B_{r-1}^{p+r, q-r}\right)\right) \\
& =\left(\delta \bmod F^{p^{\prime}+1} D\right) \cdot d_{r}\left(c \bmod \left(Z_{r-1}^{p+1, q-1}+B_{r-1}^{p, q}\right)\right) \\
& =\bar{\delta} \cdot d_{r}(\bar{c}) \in E_{r}^{p+p^{\prime}, q-p^{\prime}} .
\end{aligned}
$$

Thus, $d_{r}$ is graded $W$-linear and $\operatorname{ker}\left(d_{r}\right), \operatorname{im}\left(d_{r}\right)$, and $E_{r}=\operatorname{ker}\left(d_{r}\right) / \operatorname{im}\left(d_{r}\right)$ are graded $W$-modules.

(2) follows from (dd): As a subquotient of $E_{r}, E_{r+1}$ is Noetherian by induction. Let $\pi_{r}: \operatorname{ker}\left(d_{r}\right) \rightarrow \operatorname{ker}\left(d_{r}\right) / \operatorname{im}\left(d_{r}\right)=E_{r+1}$ denote the natural projection. Note that $\pi_{r}^{-1}\left(\operatorname{im}\left(d_{r+1}\right)\right) \supseteq \pi_{r}^{-1}(0)=\operatorname{im}\left(d_{r}\right)$. Then the chain

$$
\operatorname{im}\left(d_{0}\right) \subseteq \pi_{0}^{-1}\left(\operatorname{im}\left(d_{1}\right)\right) \subseteq \pi_{0}^{-1}\left(\pi_{1}^{-1} \operatorname{im}\left(d_{2}\right)\right) \subseteq \cdots
$$

of submodules of $E_{0}$ must stabilize at some $r_{0}: \pi_{r}^{-1}\left(\operatorname{im}\left(d_{r+1}\right)\right)=\operatorname{im}\left(d_{r}\right)=\pi_{r}^{-1}(0)$ and hence $\operatorname{im}\left(d_{r+1}\right)=0$ for all $r \geq r_{0}$.

(3) follows from (e区):

$$
\begin{aligned}
B_{\infty}^{p, q} & =F^{p} K^{p+q} \cap d\left(K^{p+q-1}\right)=F^{p} K^{p+q} \cap d\left(\bigcup_{r} F^{p-r} K^{p+q-1}\right) \\
& =\bigcup_{r}\left(F^{p} K^{p+q} \cap d\left(F^{p-r} K^{p+q-1}\right)\right)=\bigcup_{r} B_{r}^{p, q}, \\
Z_{\infty}^{p, q} & =F^{p} K^{p+q} \cap d^{-1}(0)=F^{p} K^{p+q} \cap d^{-1}\left(\bigcap_{r} F^{p+r} K^{p+q+1}\right) \\
& =\bigcap_{r}\left(F^{p} K^{p+q} \cap d^{-1}\left(F^{p+r} K^{p+q+1}\right)\right)=\bigcap_{r} Z_{r}^{p, q} .
\end{aligned}
$$

By (A.2), $d_{r}=0$ means that

$$
B_{r}^{p, q}=d\left(Z_{r}^{p-r, q+r-1}\right) \subseteq Z_{r-1}^{p+1, q-1}+B_{r-1}^{p, q} \subseteq B_{r-1}^{p, q}+F^{p+1} K^{p+q}
$$

and also

$$
d\left(Z_{r}^{p, q}\right) \subseteq Z_{r-1}^{p+r+1, q-r}+B_{r-1}^{p+r, q-r+1}=Z_{r-1}^{p+r+1, q-r}+d\left(Z_{r-1}^{p+1, q-1}\right)
$$

which implies that

$$
Z_{r}^{p, q} \subseteq Z_{r+1}^{p, q}+Z_{r-1}^{p+1, q-1} \subseteq Z_{r+1}^{p, q}+F^{p+1} K^{p+q} .
$$

From (A.4) and (A.5) follows $\operatorname{gr}^{p} B_{r}^{p, q}=\operatorname{gr}^{p} B_{r-1}^{p, q}$ and $\operatorname{gr}^{p} Z_{r}^{p, q}=\operatorname{gr}^{p} Z_{r+1}^{p, q}$ for $r \geq r_{0}$. By (A.1) and (A.3) and exactness of gr for induced filtrations, we conclude that, for $r \geq r_{0}$,

$$
E_{r}^{p, q}=\operatorname{gr}^{p} \frac{Z_{r}^{p, q}}{B_{r-1}^{p, q}}=\frac{\operatorname{gr}^{p} Z_{r}^{p, q}}{\operatorname{gr}^{p} B_{r-1}^{p, q}}=\frac{\operatorname{gr}^{p}\left(\bigcap_{r} Z_{r}^{p, q}\right)}{\operatorname{gr}^{p}\left(\bigcup_{r} B_{r-1}^{p, q}\right)}=\frac{\operatorname{gr}^{p} Z_{\infty}^{p, q}}{\operatorname{gr}^{p} B_{\infty}^{p, q}}=\operatorname{gr}^{p} \frac{Z_{\infty}^{p, q}}{B_{\infty}^{p, q}}=E_{\infty}^{p, q} .
$$


Corollary A.2. Under the hypotheses of Theorem A.1 assume that $W=\operatorname{gr}(D)$ is commutative, let $P \in \operatorname{Spec}(W)$ be a prime ideal and denote by $W_{P}$ the local ring of $\operatorname{Spec}(W)$ at $P$. Then there is a spectral sequence

$$
H^{p}\left(\operatorname{gr}\left(K^{\bullet}\right) \otimes_{W} W_{P}\right)={ }^{\prime} E_{1} \Longrightarrow{ }^{\prime} E_{\infty}=\left(\operatorname{gr}\left(H^{p}\left(K^{\bullet}\right)\right)\right) \otimes_{W} W_{P} .
$$

This spectral sequence collapses eventually and converges: ' $E_{r}={ }^{\prime} E_{r+1}={ }^{\prime} E_{\infty}$ for all large $r$.

Proof. The spectral sequence $\left(E_{r}, d_{r}\right)$ of Theorem A.1 is a sequence of $W$-modules and $W$-morphisms. Since localization commutes with the formation of kernels and cokernels, the objects and morphisms $\left({ }^{\prime} E_{r},{ }^{\prime} d_{r}\right)=\left(E_{r} \otimes_{W} W_{P}, d_{r} \otimes_{W} W_{P}\right)$ form a spectral sequence. Since $\left(E_{r}, d_{r}\right)$ collapses, so does $\left({ }^{\prime} E_{r},{ }^{\prime} d_{r}\right)$, converging to the localization of $E_{\infty}$.

Remark A.3. As far as we can see, the collapse of the sequence is essential for the corollary. If in general $\left(E_{r}, d_{r}\right)$ is a spectral sequence of $W$-modules and $W$ morphisms converging to $E_{\infty}$ in the sense that $E_{\infty}=\bigcap_{r} Z_{r} / \bigcup_{r} B_{r}$ then while $\left(\bigcup_{r} B_{r}\right) \otimes_{W} W_{P}=\bigcup_{r}\left(B_{r} \otimes_{W} W_{P}\right)$, it is not necessarily the case that $\left(\bigcap_{r} Z_{r}\right) \otimes_{W}$ $W_{P}=\bigcap_{r}\left(Z_{r} \otimes_{W} W_{P}\right)$. The problem is that infinite intersections (in contrast to infinite unions) and homomorphisms (such as the localization morphism) are not necessarily interchangeable.

In the situation of the theorem it is probably not sufficient to replace (d) by the condition that $K^{\bullet}$ consists of Noetherian $D$-modules. For example, in [ST04] some rings appear in the theory of hypergeometric systems that are Noetherian, but the order filtration leads to non-Noetherian associated graded rings.

\section{ACKNOWLEDGMENTS}

UW is grateful to Francisco Castro-Jiménez, Alicia Dickenstein, Ezra Miller, Anurag Singh and Will Traves for interesting and helpful conversations. MS wishes to thank Purdue University for their hospitality during much of the preparation of this article. We thank María-Cruz Fernández-Fernández for pointing out an error in the initial version of Lemma 2.12 .

\section{REFERENCES}

[ACJG96] A. Assi, F. J. Castro-Jiménez, and J. M. Granger, How to calculate the slopes of a D-module, Compositio Math. 104 (1996), no. 2, 107-123. MR MR1421395 (98i:32010)

[ACJG00] A. Assi, F. J. Castro-Jiménez, and M. Granger, The Gröbner fan of an $A_{n}$-module, J. Pure Appl. Algebra 150 (2000), no. 1, 27-39. MR MR1762918 (2001j:16036)

[Ado94] Alan Adolphson, Hypergeometric functions and rings generated by monomials, Duke Math. J. 73 (1994), no. 2, 269-290. MR 96c:33020

[BH93] Winfried Bruns and Jürgen Herzog, Cohen-Macaulay rings, Cambridge Studies in Advanced Mathematics, vol. 39, Cambridge University Press, Cambridge, 1993. MR MR1251956 (95h:13020)

[BS98] M. P. Brodmann and R. Y. Sharp, Local cohomology: an algebraic introduction with geometric applications, Cambridge Studies in Advanced Mathematics, vol. 60, Cambridge University Press, Cambridge, 1998. MR MR1613627 (99h:13020)

[CDS01] Eduardo Cattani, Alicia Dickenstein, and Bernd Sturmfels, Rational hypergeometric functions, Compositio Math. 128 (2001), no. 2, 217-239. MR MR1850183 (2003f:33016)

[CJT03] Francisco Jesús Castro-Jiménez and Nobuki Takayama, Singularities of the hypergeometric system associated with a monomial curve, Trans. Amer. Math. Soc. 355 (2003), no. 9, 3761-3775 (electronic). MR MR1990172 (2004j:32009) 
[CK99] David A. Cox and Sheldon Katz, Mirror symmetry and algebraic geometry, Mathematical Surveys and Monographs, vol. 68, American Mathematical Society, Providence, RI, 1999. MR MR1677117 (2000d:14048)

[Ful98] William Fulton, Intersection theory, second ed., Ergebnisse der Mathematik und ihrer Grenzgebiete. 3. Folge. A Series of Modern Surveys in Mathematics [Results in Mathematics and Related Areas. 3rd Series. A Series of Modern Surveys in Mathematics], vol. 2, Springer-Verlag, Berlin, 1998. MR MR1644323 (99d:14003)

[GGZ87] I. M. Gel'fand, M. I. Graev, and A. V. Zelevinskiǔ, Holonomic systems of equations and series of hypergeometric type, Dokl. Akad. Nauk SSSR 295 (1987), no. 1, 14-19. MR MR902936 (88j:58118)

[God58] Roger Godement, Topologie algébrique et théorie des faisceaux, Actualit'es Sci. Ind. No. 1252. Publ. Math. Univ. Strasbourg. No. 13, Hermann, Paris, 1958. MR MR0102797 (21 \#1583)

[GZK89] I. M. Gel'fand, A. V. Zelevinskiı̌, and M. M. Kapranov, Hypergeometric functions and toric varieties, Funktsional. Anal. i Prilozhen. 23 (1989), no. 2, 12-26. MR 90m:22025

[HH03] María Isabel Hartillo Hermoso, Slopes of hypergeometric systems of codimension one, Proceedings of the International Conference on Algebraic Geometry and Singularities (Spanish) (Sevilla, 2001), vol. 19, 2003, pp. 455-466. MR MR2023195 (2005d:16039)

[HH05] María Isabel Hartillo-Hermoso, Irregular hypergeometric systems associated with a singular monomial curve, Trans. Amer. Math. Soc. 357 (2005), no. 11, 4633-4646 (electronic). MR MR2156724

[HK84] R. Hotta and M. Kashiwara, The invariant holonomic system on a semisimple Lie algebra, Invent. Math. 75 (1984), no. 2, 327-358. MR MR732550 (87i:22041)

[Hot98] R. Hotta, Equivariant d-modules, arXiv.org (1998), no. RT/9805021.

[Inc44] E. L. Ince, Ordinary Differential Equations, Dover Publications, New York, 1944. MR MR0010757 (6,65f)

[Kou76] A. G. Kouchnirenko, Polyèdres de Newton et nombres de Milnor, Invent. Math. 32 (1976), no. 1, 1-31. MR MR0419433 (54 \#7454)

[Lau85] Yves Laurent, Théorie de la deuxième microlocalisation dans le domaine complexe, Progress in Mathematics, vol. 53, Birkhäuser Boston Inc., Boston, MA, 1985. MR MR776973 (86k:58113)

[Lau87] Polygône de Newton et b-fonctions pour les modules microdifférentiels, Ann. Sci. École Norm. Sup. (4) 20 (1987), no. 3, 391-441. MR MR925721 (89k:58282)

[LM99] Yves Laurent and Zoghman Mebkhout, Pentes algébriques et pentes analytiques d'un D-module, Ann. Sci. École Norm. Sup. (4) 32 (1999), no. 1, 39-69. MR MR1670595 (2001b:32015)

[Mal74] Bernard Malgrange, Sur les points singuliers des équations différentielles, Enseignement Math. (2) 20 (1974), 147-176. MR MR0368074 (51 \#4316)

[Meb89] Zoghman Mebkhout, Le théorème de comparaison entre cohomologies de de Rham d'une variété algébrique complexe et le théorème d'existence de Riemann, Inst. Hautes Études Sci. Publ. Math. (1989), no. 69, 47-89. MR MR1019961 (91a:32016)

[MMW05] Laura Felicia Matusevich, Ezra Miller, and Uli Walther, Homological methods for hypergeometric families, J. Amer. Math. Soc. 18 (2005), no. 4, 919-941 (electronic). MR MR2163866

[MS05] Ezra Miller and Bernd Sturmfels, Combinatorial commutative algebra, Graduate Texts in Mathematics, vol. 227, Springer-Verlag, New York, 2005. MR MR2110098 (2006d:13001)

[OT01] Peter Orlik and Hiroaki Terao, Arrangements and hypergeometric integrals, MSJ Memoirs, vol. 9, Mathematical Society of Japan, Tokyo, 2001. MR MR1814008 (2003a:32048)

[Sch85] Pierre Schapira, Microdifferential systems in the complex domain, Grundlehren der Mathematischen Wissenschaften [Fundamental Principles of Mathematical Sciences], vol. 269, Springer-Verlag, Berlin, 1985. MR MR774228 (87k:58251)

[Ser65] Jean-Pierre Serre, Algèbre locale. Multiplicités, Cours au Collège de France, 19571958, rédigé par Pierre Gabriel. Seconde édition, 1965. Lecture Notes in Mathematics, vol. 11, Springer-Verlag, Berlin, 1965. MR MR0201468 (34 \#1352)

[Smi01] Gregory G. Smith, Irreducible components of characteristic varieties, J. Pure Appl. Algebra 165 (2001), no. 3, 291-306. MR MR1864474 (2003a:16034) 
[SST00] Mutsumi Saito, Bernd Sturmfels, and Nobuki Takayama, Gröbner deformations of hypergeometric differential equations, Algorithms and Computation in Mathematics, vol. 6, Springer-Verlag, Berlin, 2000. MR 2001i:13036

[ST04] Mutsumi Saito and William N. Traves, Finite generation of rings of differential operators of semigroup algebras, J. Algebra 278 (2004), no. 1, 76-103. MR MR2068067 (2005e:16041)

[Stu96] Bernd Sturmfels, Gröbner bases and convex polytopes, University Lecture Series, vol. 8, American Mathematical Society, Providence, RI, 1996. MR MR1363949 (97b:13034)

M. Schulze, Oklahoma State University, Dept. of Mathematics, 401 MSCS, StillwaTER, OK 74078, USA

E-mail address: mschulze@math.okstate.edu

U. Walther, Purdue University, Dept. of Mathematics, 150 N. University St., West LAFAYETTE, IN 47907, USA

E-mail address: walther@math.purdue.edu 